\title{
Orthogonally Functionalized Oligomers for Controlled
}

\author{
Self-Assembly
}

\section{Table of Contents for Supporting Information}

$\begin{array}{ll}\text { General Experimental procedures } & \text { S3 }\end{array}$

General Procedure for Castro-Stephens/Sonogashira Coupling $\quad$ S3

General Procedure for the Formation of Self-Assembled Monolayers $\quad$ S3

$\begin{array}{ll}\text { Experimental for compound } 7 & \text { S4 }\end{array}$

$\begin{array}{lr}\text { Experimental for compound } \mathbf{8} & \text { S5 }\end{array}$

$\begin{array}{ll}\text { Experimental for compound } \mathbf{9}^{3} & \text { S5 }\end{array}$

$\begin{array}{ll}\text { Experimental for compound } \mathbf{1 0} & \text { S6 }\end{array}$

$\begin{array}{ll}\text { Experimental for compound } \mathbf{1 1} & \text { S6 }\end{array}$

$\begin{array}{ll}\text { Experimental for compound } \mathbf{1 2} & \text { S7 }\end{array}$

$\begin{array}{ll}\text { Experimental for compound } \mathbf{1 3} & \text { S8 }\end{array}$

$\begin{array}{ll}\text { Experimental for compound } \mathbf{1 4} & \text { S8 }\end{array}$

$\begin{array}{lr}\text { Experimental for compound } \mathbf{1 5} & \text { S9 }\end{array}$

$\begin{array}{lr}\text { Experimental for compound } \mathbf{1 6} & \text { S9 }\end{array}$

$\begin{array}{ll}\text { Experimental for compound } \mathbf{1 7} & \text { S10 }\end{array}$

$\begin{array}{ll}\text { Experimental for compound } \mathbf{1 8} & \mathrm{S} 10\end{array}$

$\begin{array}{ll}\text { Experimental for compound } 19 & \text { S11 }\end{array}$

$\begin{array}{ll}\text { Experimental for compound } \mathbf{2 0} & \text { S11 }\end{array}$

$\begin{array}{ll}\text { Experimental for compound } \mathbf{2 1}^{13} & \mathrm{~S} 12\end{array}$

$\begin{array}{ll}\text { Experimental for compound } \mathbf{2 2}^{14} & \mathrm{~S} 12\end{array}$

$\begin{array}{ll}\text { Experimental for compound } \mathbf{2 3}^{15} & \mathrm{~S} 13\end{array}$ 
Flatt, Yao, Maya, and Tour, Orthogonally Functionalized Oligomers, Supporting Information

$\begin{array}{lr}\text { Experimental for compound } \mathbf{2 4} & \text { S13 }\end{array}$

$\begin{array}{lr}\text { Experimental for compound } 25 & \text { S14 }\end{array}$

$\begin{array}{lr}\text { Experimental for compound } \mathbf{2 6}^{16} & \text { S14 }\end{array}$

$\begin{array}{ll}\text { Experimental for compound } 27 & \text { S15 }\end{array}$

$\begin{array}{lr}\text { Experimental for compound } \mathbf{2 8} & \mathrm{S} 15\end{array}$

$\begin{array}{lr}{ }^{1} \mathrm{H} \text { and }{ }^{13} \mathrm{C} \text { NMR Spectra } & \text { S16 }\end{array}$

$1 \quad$ S17

$\begin{array}{ll}2 & \text { S19 }\end{array}$

$3 \quad$ S21

$4 \quad$ S23

$5 \quad$ S25

$\begin{array}{ll}6 & \text { S27 }\end{array}$

$\begin{array}{ll}7 & \text { S29 }\end{array}$

$8 \quad$ S31

92 S33

$10 \quad$ S35

$\begin{array}{ll}11 & \text { S37 }\end{array}$

$12 \quad$ S39

$14+$ S41

15 S43

$16 \quad$ S45

$17 \quad$ S47

$\begin{array}{ll}18 & \text { S49 }\end{array}$

$19-$ S51

$20 \quad$ S53

$24 \quad$ S55

$25 \quad$ S57

$\begin{array}{ll}27 & \text { S59 }\end{array}$ 
Flatt, Yao, Maya, and Tour, Orthogonally Functionalized Oligomers, Supporting Information

References

General: All reactions were performed under a dry atmosphere of $\mathrm{N}_{2}$ unless otherwise stated. Reagent grade tetrahydrofuran (THF) and diethyl ether $\left(\mathrm{Et}_{2} \mathrm{O}\right)$ were distilled under $\mathrm{N}_{2}$ over sodium benzophenone ketyl. Silica gel plugs and flash chromatography were carried out on silica gel (230-400 mesh from EM science). Thin layer chromatography was carried out on glass plates coated with silica gel $40 \mathrm{~F}_{254}$ with a layer thickness of $0.25 \mathrm{~mm}$ purchased from EM Science. All new compounds were named using the Beilstein Autonom feature of Beilstein Commander.

\section{General procedure for coupling a terminal alkyne with an aryl halide (Castro-} Stephens/Sonogashira protocol). ${ }^{19}$ To an oven dried flask containing a magnetic stir bar was added the aryl halide, bis(triphenylphosphine)palladium(II) dichloride ( $5 \mathrm{~mol} \%$ based on aryl halide), and copper(I) iodide (10 mol \% based on aryl halide). Alternatively, $\mathrm{Pd}(\mathrm{dba})_{2}(5 \mathrm{~mol} \%$ based on aryl halide), $\mathrm{PPh}_{3}(20 \mathrm{~mol} \%)$, and copper(I) iodide (10 mol \%) was used. The vessel was then sealed with a rubber septum, evacuated and backfilled with $\mathrm{N}_{2}(3 \times)$. A co-solvent of THF was added followed by the amine base. The terminal alkyne was added and the reaction was heated, if necessary, until the aryl halide was consumed as judged by TLC. The reaction vessel was cooled to room temperature and quenched with water. The organic layer was diluted with $\mathrm{CH}_{2} \mathrm{Cl}_{2}$ and washed with a saturated solution of $\mathrm{NH}_{4} \mathrm{Cl}$. The organic layer was dried using anhydrous $\mathrm{MgSO}_{4}$ and the solvent was removed in vacuo. The crude product was then purified by flash chromatography (silica gel).

General procedure for the formation of self-assembled monolayers (SAMs). The gold surface was evaporated and cleaned according to established procedures. ${ }^{5}$ The compound $(2 \mathrm{mg})$ was added to a 20 
Flatt, Yao, Maya, and Tour, Orthogonally Functionalized Oligomers, Supporting Information $\mathrm{mL}$ vial followed by $10 \mathrm{~mL}$ of freshly distilled THF. The gold substrate was added and allowed to incubate in the dark for $40 \mathrm{~min}$ at room temperature. The substrate was removed, rinsed with anhydrous EtOH, and dried with $\mathrm{N}_{2}$. Monolayer thickness was determined on a Gaertner-LSE ellipsometer. Measurements were performed immediately before and immediately after monolayer formation. Thicknesses were calculated based on the refractive index $n_{\mathrm{f}}=1.5$ for compound 4 and $n_{\mathrm{f}}=$ 1.55 for compounds 1 and 2 ( $k_{\mathrm{f}}=0$ for all compounds).

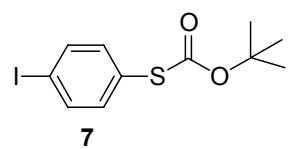

Thiocarbonic acid $\boldsymbol{O}$-tert-butyl ester $\boldsymbol{S}$-(4-iodo-phenyl) ester (7). To a solution of 1,4diiodobenzene $(4.00 \mathrm{~g}, 12.13 \mathrm{mmol})$ in THF $(50 \mathrm{~mL})$ at $-78{ }^{\circ} \mathrm{C}$ was slowly added tert-butyllithium (14.3 $\mathrm{mL}, 24.25 \mathrm{mmol}, 1.7 \mathrm{M}$ in pentane). The resulting white slurry was stirred for 30 min and sulfur $(0.43 \mathrm{~g}, 13.34 \mathrm{mmol})$ was added in one portion. The mixture was then allowed to warm to $0{ }^{\circ} \mathrm{C}$ for 10 $15 \mathrm{~min}$. The clear solution was cooled back to $-78^{\circ} \mathrm{C}$ and a solution of di-(tert-butyl)dicarbonate $(3.18$ $\mathrm{g}, 14.55 \mathrm{mmol})$ in THF $(20 \mathrm{~mL})$ was added via cannula. The reaction was allowed to warm to room temperature and poured into water. The mixture was extracted with EtOAc $(1 \times)$ and the organic layer was washed with $\mathrm{H}_{2} \mathrm{O}(3 \times)$, brine $(1 \times)$, and dried over anhydrous $\mathrm{MgSO}_{4}$. Flash chromatography, silica gel (4:1 hexane $\left./ \mathrm{CH}_{2} \mathrm{Cl}_{2}\right)$ gave 7 as colorless oil that slowly crystallized $(2.91 \mathrm{~g}, 71 \%)$. Mp: 58 $60{ }^{\circ} \mathrm{C}$. FTIR (KBr) 2976, 1717, 1565, 1469, 1365, 1220, 1201, 1128, 1090, 1005, 848, 835, 812, 673, $530 \mathrm{~cm}^{-1} .{ }^{1} \mathrm{H}$ NMR $\left(400 \mathrm{MHz}, \mathrm{CDCl}_{3}\right) \delta 7.72(\mathrm{~d}, J=8.3 \mathrm{~Hz}, 2 \mathrm{H}), 7.25(\mathrm{~d}, J=8.3 \mathrm{~Hz}, 2 \mathrm{H}), 1.51(\mathrm{~s}$, 9H). ${ }^{13} \mathrm{C} \mathrm{NMR}\left(100 \mathrm{MHz}, \mathrm{CDCl}_{3}\right) \delta 167.1,138.3,136.4,128.7,95.9,86.1,28.3$. HRMS calc'd for $\mathrm{C}_{11} \mathrm{H}_{13} \mathrm{IO}_{2} \mathrm{~S}: 335.9681$. Found: 335.9684. 
Flatt, Yao, Maya, and Tour, Orthogonally Functionalized Oligomers, Supporting Information

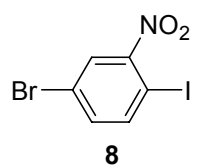

4-Bromo-1-iodo-2-nitrobenzene (8). To a $500 \mathrm{~mL}$ round bottom containing a stir bar was added borontrifluoride diethyletherate $(9.95 \mathrm{~mL}, 78.53 \mathrm{mmol})$ and the flask was cooled to $-30{ }^{\circ} \mathrm{C}$. 4 -Bromo2-nitroaniline ${ }^{20}(4.05 \mathrm{~g}, 18.67 \mathrm{mmol})$ dissolved in THF $(50 \mathrm{~mL})$ was added dropwise. $90 \%$ tertbutylnitrite $(8.17 \mathrm{~mL}, 68.72 \mathrm{mmol})$ was dissolved in THF $(50 \mathrm{~mL})$ and added dropwise. The reaction was allowed to warm to $-5{ }^{\circ} \mathrm{C}$ at which time $100 \mathrm{~mL}$ of diethyl ether was added and the mixture was allowed to stir at $-5{ }^{\circ} \mathrm{C}$ for 10 min until a pale solid precipitated. The solid was filtered and washed with ether to afford a pale solid which was then added in one portion to a $500 \mathrm{~mL}$ round bottom containing a stir bar, potassium iodide $(4.40 \mathrm{~g}, 26.53 \mathrm{mmol})$, iodine $(3.37 \mathrm{~g}, 13.27 \mathrm{mmol})$, and acetonitrile $(75 \mathrm{~mL})$. The reaction was allowed to stir at room temperature for $15 \mathrm{~min}$. $150 \mathrm{~mL}$ of a saturated aqueous solution of sodium thiosulfate was added as well as $150 \mathrm{~mL}$ of $\mathrm{CH}_{2} \mathrm{Cl}_{2}$. The mixture was allowed to stir for $5 \mathrm{~min}$, the layers were separated, the organics were dried using anhydrous $\mathrm{MgSO}_{4}$, and the solvent was removed in vacuo. This afforded the product as a yellow solid (3.8 $\mathrm{g}$, 62\%). Mp: $87-89^{\circ} \mathrm{C}$. FTIR (KBr) 3085, 1573, 1553, 1528, 1453, 1346, 1271, 1250, 1152, 1090, 1019, $886,872,818,752,737 \mathrm{~cm}^{-1} .{ }^{1} \mathrm{H}$ NMR $\left(400 \mathrm{MHz}, \mathrm{CDCl}_{3}\right) \delta 8.00(\mathrm{~d}, J=2.8 \mathrm{~Hz}, 1 \mathrm{H}), 7.89(\mathrm{~d}, J=8.6$, $1 \mathrm{H}), 7.40(\mathrm{dd}, J=8.6,2.8 \mathrm{~Hz}, 1 \mathrm{H}) .{ }^{13} \mathrm{C} \mathrm{NMR}\left(100 \mathrm{MHz}, \mathrm{CDCl}_{3}\right) \delta 153.9,143.3,136.9,128.9,123.1$, 84.9. HRMS calc'd for $\mathrm{C}_{6} \mathrm{H}_{3} \mathrm{BrINO}_{2}$ : 326.8392 . Found: 326.8389 .

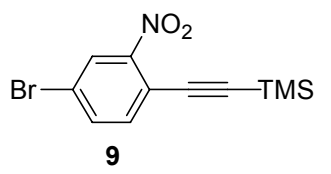

(4-Bromo-2-nitro-phenylethynyl)-trimethyl-silane (9). ${ }^{3}$ See the general coupling procedure. Used were 8 (3.67 g, $11.19 \mathrm{mmol})$, trimethylsilylacetylene $(1.58 \mathrm{~mL}, 11.19 \mathrm{mmol}), \mathrm{PdCl}_{2}\left(\mathrm{PPh}_{3}\right)_{2}(0.23 \mathrm{~g}$, $0.33 \mathrm{mmol}), \mathrm{CuI}(0.13 \mathrm{~g}, 0.67 \mathrm{mmol})$, THF $(20 \mathrm{~mL})$, and triethylamine $(5 \mathrm{~mL})$. Flash chromatography, silica gel (1:1 $\mathrm{CH}_{2} \mathrm{Cl}_{2}$ /hexanes) afforded the product as a yellow solid (3.16 g, 95\%). Mp: $30{ }^{\circ} \mathrm{C}$. FTIR 
Flatt, Yao, Maya, and Tour, Orthogonally Functionalized Oligomers, Supporting Information (KBr) 3093, 2959, 2164, 1599, 1553, 1531, 1469, 1409, 1341, 1280, 1250, 1216, 1152, 1092, 886, 844, $759,716,653,545 \mathrm{~cm}^{-1} .{ }^{1} \mathrm{H}$ NMR $\left(400 \mathrm{MHz} \mathrm{CDCl}_{3}\right) \delta 8.15(\mathrm{~d}, J=2.1 \mathrm{~Hz}, 1 \mathrm{H}), 7.67(\mathrm{dd}, J=8.8,2.1$ $\mathrm{Hz}, 1 \mathrm{H}), 7.50(\mathrm{~d}, J=8.8 \mathrm{~Hz}, 1 \mathrm{H}), 0.27(\mathrm{~s}, 9 \mathrm{H}) .{ }^{13} \mathrm{C} \mathrm{NMR}\left(100 \mathrm{MHz}, \mathrm{CDCl}_{3}\right) \delta 150.7,136.4,136.2$, 128.0, 122.6, 117.7, 105.8, 98.8, 0.0. HRMS calc'd for $\mathrm{C}_{11} \mathrm{H}_{12} \mathrm{BrNO}_{2} \mathrm{Si}$ : 296.9802. Found: 296.9800.

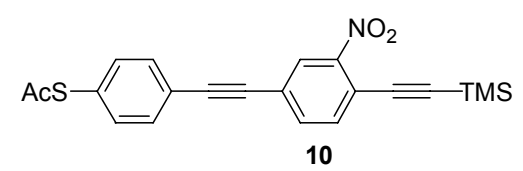

Thioacetic acid $S$-[4-(3-nitro-4-trimethylsilanylethynyl-phenylethynyl)-phenyl] ester (10). See the general coupling procedure. Used were 9 (1.50 g, $5.03 \mathrm{mmol})$, 1-ethynyl-4-thioacetylbenzene ${ }^{4}(0.74 \mathrm{~g}$, $4.19 \mathrm{mmol}), \mathrm{PdCl}_{2}\left(\mathrm{PPh}_{3}\right)_{2}(0.18 \mathrm{~g}, 0.25 \mathrm{mmol}), \mathrm{CuI}(0.10 \mathrm{~g}, 0.50 \mathrm{mmol})$, THF $(20 \mathrm{~mL})$, and triethylamine $(4 \mathrm{~mL})$. Flash chromatography, silica gel $\left(1: 1\right.$ hexane $\left./ \mathrm{CH}_{2} \mathrm{Cl}_{2}\right)$ afforded the desired product as a yellow solid (0.48 g, 29\%). Mp: 104-107 ${ }^{\circ} \mathrm{C}$. FTIR (KBr) 2961, 2206, 2155, 1699, 1588, $1536,1520,1499,1396,1351,1249,1223,1180,1131,1113,1089,1013,946,909,850,826,759$, 741, 696, 638, 623, 592, $542 \mathrm{~cm}^{-1} .{ }^{1} \mathrm{H} \mathrm{NMR}\left(400 \mathrm{MHz}, \mathrm{CDCl}_{3}\right) \delta 8.16(\mathrm{~d}, J=1.5 \mathrm{~Hz}, 1 \mathrm{H}), 7.67(\mathrm{dd}, J$ $=8.1,1.5 \mathrm{~Hz}, 1 \mathrm{H}), 7.63(\mathrm{~d}, J=8.1 \mathrm{~Hz}, 1 \mathrm{H}), 7.57(\mathrm{~d}, J=8.6 \mathrm{~Hz}, 2 \mathrm{H}), 7.44(\mathrm{~d}, J=8.6 \mathrm{~Hz}, 2 \mathrm{H}), 2.46(\mathrm{~s}$, 3H), $0.30(\mathrm{~s}, 9 \mathrm{H}) .{ }^{13} \mathrm{C}$ NMR $\left(100 \mathrm{MHz}, \mathrm{CDCl}_{3}\right) \delta 193.1,150.1,135.22,135.15,134.4,132.4,129.5$, 127.5, 124.1, 123.2, 118.0, 106.1, 99.3, 92.9, 88.3, 30.4, -0.3. HRMS calc'd for $\mathrm{C}_{21} \mathrm{H}_{19} \mathrm{NO}_{3} \mathrm{Ssi}_{\text {: }}$ 393.0855. Found: 393.0854 .

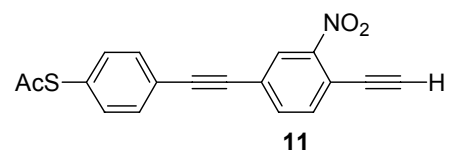

Thioacetic acid $S$-[4-(4-ethynyl-3-nitro-phenylethynyl)-phenyl] ester (11). To a $100 \mathrm{~mL}$ round bottom containing a stir bar was added $10(0.43 \mathrm{~g}, 1.09 \mathrm{mmol})$, THF $(10 \mathrm{~mL})$, acetic anhydride $(0.21$ $\mathrm{mL}, 2.19 \mathrm{mmol})$, acetic acid $(0.13 \mathrm{~mL}, 2.19 \mathrm{mmol})$, and tetrabutylammonium fluoride $(1.31 \mathrm{~mL}$ of a 1.0 $\mathrm{M}$ solution in THF). The reaction was allowed to stir at room temperature for $15 \mathrm{~min}$, poured into water and extracted with $\mathrm{CH}_{2} \mathrm{Cl}_{2}(3 \times)$. The organic layer was dried using anhydrous $\mathrm{MgSO}_{4}$ and the 
Flatt, Yao, Maya, and Tour, Orthogonally Functionalized Oligomers, Supporting Information solvent was removed in vacuo to give the product as an orange solid (0.29 g, 84\%). Mp: $143-145{ }^{\circ} \mathrm{C}$. FTIR (KBr) 3277, 1695, 1540, 1524, 1352, 1112, 839, 829, $625 \mathrm{~cm}^{-1} .{ }^{1} \mathrm{H}$ NMR $\left(400 \mathrm{MHz}, \mathrm{CDCl}_{3}\right) \delta$ $8.18(\mathrm{~d}, J=1.1 \mathrm{~Hz}, 1 \mathrm{H}), 7.69(\mathrm{dd}, J=8.1,1.5 \mathrm{~Hz}, 1 \mathrm{H}), 7.66(\mathrm{~d}, J=8.1 \mathrm{~Hz}, 1 \mathrm{H}), 7.57(\mathrm{~d}, J=8.5 \mathrm{~Hz}$, 2H), $7.43(\mathrm{~d}, J=8.5 \mathrm{~Hz}, 2 \mathrm{H}), 3.62(\mathrm{~s}, 1 \mathrm{H}), 2.46(\mathrm{~s}, 3 \mathrm{H}) .{ }^{13} \mathrm{C} \mathrm{NMR}\left(125 \mathrm{MHz}, \mathrm{CDCl}_{3}\right) \delta 193.3,150.4$, $135.7,135.5,134.5,132.5,129.6,127.7,124.9,123.2,117.1,93.2,88.2,87.1,78.5,30.5$. HRMS calc'd for $\mathrm{C}_{18} \mathrm{H}_{11} \mathrm{NO}_{3} \mathrm{~S}$ : 321.0460 . Found: 321.0456 .

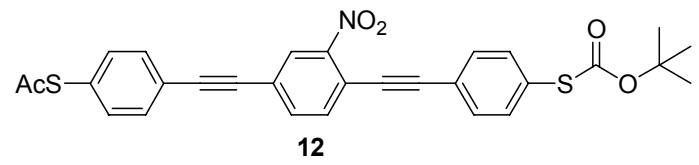

Thioacetic acid $S$-\{4-[4-(4-tert-butoxycarbonylsulfanyl-phenylethynyl)-3-nitro-phenylethynyl]phenyl\} ester (12). See the general coupling procedure. Used were 11 (0.95 g, $2.96 \mathrm{mmol}), 7$ (1.09 g, $3.25 \mathrm{mmol}), \mathrm{Pd}(\mathrm{dba})_{2}(0.17 \mathrm{~g}, 0.30 \mathrm{mmol}), \mathrm{PPh}_{3}(0.31 \mathrm{~g}, 1.2 \mathrm{mmol}), \mathrm{CuI}(0.11 \mathrm{~g}, 0.60 \mathrm{mmol}), \mathrm{THF}$ (30 $\mathrm{mL})$ and $i-\operatorname{Pr}_{2} \mathrm{NEt}(2.1 \mathrm{~mL}, 12 \mathrm{mmol})$. The reaction was stirred at room temperature overnight. Flash chromatography, silica gel $\left(6: 3: 1\right.$ hexane $\left./ \mathrm{CH}_{2} \mathrm{Cl}_{2} / \mathrm{Et}_{2} \mathrm{O}\right)$ gave an oily residue. The oil was redissolved in $\mathrm{Et}_{2} \mathrm{O}$ and diluted with hexane. The solution was carefully concentrated to remove most of the $\mathrm{Et}_{2} \mathrm{O}$. The formed precipitate was filtered to give 12 as an orange solid (0.93 g, 59\%). Mp: $106-112{ }^{\circ} \mathrm{C}$. FTIR (KBr) 2982, 2209, 1713, 1536, 1502, 1396, 1349, 1198, 1124, 1078, 1012, 827, 618, $524 \mathrm{~cm}^{-1}$. ${ }^{1} \mathrm{H}$ NMR $\left(400 \mathrm{MHz}, \mathrm{CDCl}_{3}\right) \delta 8.23(\mathrm{~d}, J=2.0 \mathrm{~Hz}, 1 \mathrm{H}), 7.71(\mathrm{dd}, J=8.6,1.5 \mathrm{~Hz}, 1 \mathrm{H}), 7.69(\mathrm{~d}, J=8.6$ $\mathrm{Hz}, 1 \mathrm{H}), 7.60(\mathrm{~d}, J=8.6 \mathrm{~Hz}, 2 \mathrm{H}), 7.57(\mathrm{~d}, J=8.5 \mathrm{~Hz}, 2 \mathrm{H}), 7.55(\mathrm{~d}, J=8.6 \mathrm{~Hz}, 2 \mathrm{H}), 7.45(\mathrm{~d}, J=8.5$ $\mathrm{Hz}, 2 \mathrm{H}), 2.46$ (s, 3H), $1.53(\mathrm{~s}, 9 \mathrm{H}) .{ }^{13} \mathrm{C} \mathrm{NMR}\left(100 \mathrm{MHz}, \mathrm{CDCl}_{3}\right) \delta 193.3,167.1,149.6,135.5,134.8$, $134.7,134.5,132.6,132.5,130.6,129.5,127.9,124.1,123.35,123.27,118.2,98.3,93.0,88.5,86.4$, 86.3, 30.5, 28.4. HRMS calc'd for $\mathrm{C}_{29} \mathrm{H}_{23} \mathrm{NO}_{5} \mathrm{~S}_{2}$ : 529.1018. Found: 529.1018.

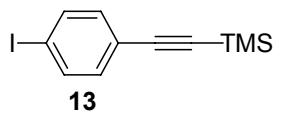


Flatt, Yao, Maya, and Tour, Orthogonally Functionalized Oligomers, Supporting Information (4-Iodo-phenylethynyl)-trimethyl-silane (13). ${ }^{10}$ To a $500 \mathrm{~mL}$ round bottom flask containing a stir bar was added 4-(bromo-phenylethynyl)-trimethyl-silane ${ }^{3}$ (3.58 g, $\left.14.14 \mathrm{mmol}\right)$. THF (40 mL) was added and the mixture was cooled to $-78{ }^{\circ} \mathrm{C}$. tert-Butyllithium $(18.24 \mathrm{~mL}, 28.28 \mathrm{mmol}$ of a $1.55 \mathrm{M}$ solution in pentane) was added dropwise and the reaction was allowed to stir at $-78{ }^{\circ} \mathrm{C}$ for 45 min at which time iodine $(4.31 \mathrm{~g}, 16.97 \mathrm{mmol})$ dissolved in THF $(50 \mathrm{~mL})$ at $-78^{\circ} \mathrm{C}$ was added. The reaction mixture was then allowed to stir at $-78^{\circ} \mathrm{C}$ for $10 \mathrm{~min}$ followed by stirring at $0{ }^{\circ} \mathrm{C}$ for $25 \mathrm{~min}$. The reaction was poured into an aqueous solution of sodium thiosulfate, extracted with $\mathrm{CH}_{2} \mathrm{Cl}_{2}(3 \times)$, dried using anhydrous $\mathrm{MgSO}_{4}$ and the solvent removed in vacuo. Flash chromatography, silica gel (1:3 $\mathrm{CH}_{2} \mathrm{Cl}_{2}$ /hexanes) afforded to product as a pale solid (4.04 g, 95\%). Mp: $52-54{ }^{\circ} \mathrm{C} . \quad{ }^{1} \mathrm{H}$ NMR (400 $\left.\mathrm{MHz}, \mathrm{CDCl}_{3}\right) \delta 7.64(\mathrm{~d}, J=8.4 \mathrm{~Hz}, 2 \mathrm{H}), 7.19(\mathrm{~d}, J=8.4 \mathrm{~Hz}, 2 \mathrm{H}), 0.25(\mathrm{~s}, 9 \mathrm{H})$.

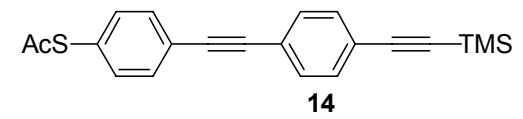

Thioacetic acid $S$-[4-(4-trimethylsilanylethynyl-phenylethynyl)-phenyl] ester (14). See the general coupling procedure. Used were 13 (2.00 g, $6.67 \mathrm{mmol})$, 1-ethynyl-4-thioacetylbenzene ${ }^{4}(1.29 \mathrm{~g}, 7.33$ $\mathrm{mmol}), \mathrm{PdCl}_{2}\left(\mathrm{PPh}_{3}\right)_{2}(0.23 \mathrm{~g}, 0.33 \mathrm{mmol}), \mathrm{CuI}(0.13 \mathrm{~g}, 0.67 \mathrm{mmol}), \mathrm{THF}(30 \mathrm{~mL})$, and triethylamine $(5$ $\mathrm{mL})$. Flash chromatography, silica gel $\left(1: 1 \mathrm{CH}_{2} \mathrm{Cl}_{2} /\right.$ hexanes $)$ afforded the product as an orange solid (1.40 g, 60\%). Mp: $113-116^{\circ} \mathrm{C}$. FTIR (KBr) 2970, 2150, 1737, 1713, 1694, 1598, 1502, 1406, 1365 , $1247,1220,1135,1116,1091,1011,950,841,758,722,698,641,625,590,540 \mathrm{~cm}^{-1} .{ }^{1} \mathrm{H}$ NMR (400 $\left.\mathrm{MHz}, \mathrm{CDCl}_{3}\right) \delta 7.55(\mathrm{~d}, J=5.1 \mathrm{~Hz}, 2 \mathrm{H}), 7.46(\mathrm{~m}, 4 \mathrm{H}), 7.40(\mathrm{~d}, J=5.1 \mathrm{~Hz}, 2 \mathrm{H}), 2.45(\mathrm{~s}, 3 \mathrm{H}), 0.27(\mathrm{~s}$, 9H). ${ }^{13} \mathrm{C} \mathrm{NMR}\left(100 \mathrm{MHz}, \mathrm{CDCl}_{3}\right) \delta 193.8,134.6,132.6,132.3,131.9,128.7,124.7,123.6,123.3$, 105.0, 96.7, 91.1, 90.9, 30.7, 0.3. HRMS calc'd for $\mathrm{C}_{21} \mathrm{H}_{20} \mathrm{OSSi}$ : 348.1004. Found: 348.1002.

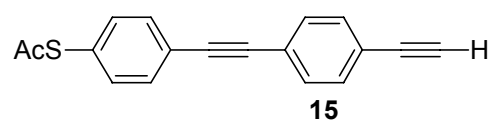


Flatt, Yao, Maya, and Tour, Orthogonally Functionalized Oligomers, Supporting Information

Thioacetic acid $S$-\{4-[4-(4-tert-butoxycarbonylsulfanyl-phenylethynyl)-phenylethynyl]-phenyl\}

ester (15). To a $100 \mathrm{~mL}$ round bottom flask containing a stir bar was added 14 (1.21 g, $3.47 \mathrm{mmol})$, THF $(20 \mathrm{~mL})$, acetic anhydride $(0.66 \mathrm{~mL}, 6.95 \mathrm{mmol})$, acetic acid $(0.40 \mathrm{~mL}, 6.95 \mathrm{mmol})$ and tetrabutylammonium fluoride $(4.17 \mathrm{~mL}$ of a $1.0 \mathrm{M}$ solution in THF). The reaction was allowed to stir at room temperature for $20 \mathrm{~min}$, poured into water and extracted with $\mathrm{CH}_{2} \mathrm{Cl}_{2}(3 \times)$. The organics were dried using anhydrous $\mathrm{MgSO}_{4}$ and the solvent was removed in vacuo to afford the product as a white solid (0.71 g, 74\%). Mp: 127-130 ${ }^{\circ} \mathrm{C}$. FTIR (KBr) 3272, 1696, 1509, 1493, 1125, 1104, 1090, 965, 832, 687, 615, 550, $529 \mathrm{~cm}^{-1} .{ }^{1} \mathrm{H}$ NMR (400 MHz, $\left.\mathrm{CDCl}_{3}\right) \delta 7.57(\mathrm{~d}, J=8.5 \mathrm{~Hz}, 2 \mathrm{H}), 7.49(\mathrm{~s}, 4 \mathrm{H})$, $7.42(\mathrm{~d}, J=8.5 \mathrm{~Hz}, 2 \mathrm{H}) 3.20(\mathrm{~s}, 1 \mathrm{H}), 2.45(\mathrm{~s}, 3 \mathrm{H}) .{ }^{13} \mathrm{C} \mathrm{NMR}\left(100 \mathrm{MHz}, \mathrm{CDCl}_{3}\right) \delta 193.8,134.7,132.6$, 132.5, 132.0, 128.8, 124.6, 123.8, 122.6, 91.0, 90.9, 83.6, 79.5, 30.7. HRMS calc'd for $\mathrm{C}_{18} \mathrm{H}_{12} \mathrm{OS}$ : 276.0609. Found: 276.0603.

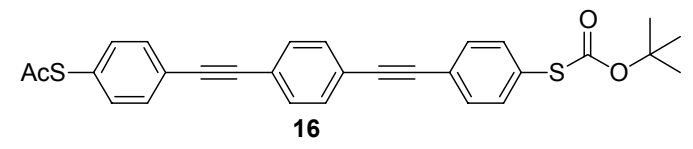

Thioacetic acid $S$-\{4-[4-(4-tert-butoxycarbonylsulfanyl-phenylethynyl)-phenylethynyl]-phenyl\} ester (16). See the general coupling procedure. Used were 15 (0.65 g, $2.35 \mathrm{mmol}), 7(0.87 \mathrm{~g}, 2.59$ $\mathrm{mmol}), \mathrm{PdCl}_{2}\left(\mathrm{PPh}_{3}\right)_{2}(0.08 \mathrm{~g}, 0.12 \mathrm{mmol}), \mathrm{CuI}(0.05 \mathrm{~g}, 0.24 \mathrm{mmol})$, THF (20 mL), and triethylamine (3 $\mathrm{mL})$. Flash chromatography, silica gel $\left(2: 1 \mathrm{CH}_{2} \mathrm{Cl}_{2} /\right.$ hexanes $)$ afforded the product as a pale solid $(0.64$ g, 56\%). Mp: $149-153^{\circ} \mathrm{C}$. FTIR (KBr) 2980, 1726, 1698, 1512, 1479, 1406, 1396, 1369, 1357, 1196, $1121,1086,1013,951,839,739,691,669,622,599,541,521,507,449 \mathrm{~cm}^{-1} .{ }^{1} \mathrm{H}$ NMR $(400 \mathrm{MHz}$, $\left.\mathrm{CDCl}_{3}\right) \delta 7.56-7.51(\mathrm{~m}, 10 \mathrm{H}), 7.43(\mathrm{~d}, J=8.7 \mathrm{~Hz}, 2 \mathrm{H}), 2.45(\mathrm{~s}, 3 \mathrm{H}), 1.53(\mathrm{~s}, 9 \mathrm{H}) .{ }^{13} \mathrm{C} \mathrm{NMR}(100 \mathrm{MHz}$, $\left.\mathrm{CDCl}_{3}\right) \delta 193.6,167.4,134.7,134.4,132.4,132.2,131.8,129.3,128.5,124.4,124.2,123.3,123.2$, 90.9, 90.8, 86.2, 30.5, 28.4. HRMS calc'd for $\mathrm{C}_{29} \mathrm{H}_{24} \mathrm{O}_{3} \mathrm{~S}_{2}$ : 484.1167. Found: 484.1161.

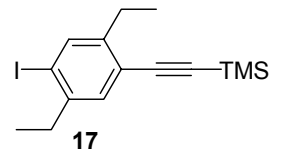


Flatt, Yao, Maya, and Tour, Orthogonally Functionalized Oligomers, Supporting Information (2,5-Diethyl-4-iodo-phenylethynyl)-trimethyl-silane (17). See the general coupling procedure. Used were 1,4-diethyl-2,5-diiodo-benzene ${ }^{3}(6.60 \mathrm{~g}, 17.10 \mathrm{mmol})$, trimethylsilylacetylene $(2.4 \mathrm{~mL}, 16.98$ $\mathrm{mmol}), \mathrm{PdCl}_{2}\left(\mathrm{PPh}_{3}\right)_{2}(0.24 \mathrm{~g}, 0.34 \mathrm{mmol}), \mathrm{CuI}(0.13 \mathrm{~g}, 0.68 \mathrm{mmol}), \mathrm{THF}(30 \mathrm{~mL})$, and $i-\mathrm{Pr}_{2} \mathrm{NEt}(9.6$ $\mathrm{mL}$ ). The reaction was allowed to stir overnight at room temperature. Purification by flash chromatography (hexanes) afforded the desired product $(2.3 \mathrm{~g}, 39 \%)$ as a light yellow oil. IR (KBr) 2965, 2872, 2299, 2152, 2054, 2007, 1941, 1860, 1763, 1588, 1467, 1374, 1328, 1249, 1140, 1061, 849, 761, 701, $662 \mathrm{~cm}^{-1} .{ }^{1} \mathrm{H}$ NMR (400 MHz, $\left.\mathrm{CDCl}_{3}\right) \delta 7.67(\mathrm{~s}, 1 \mathrm{H}), 7.28(\mathrm{~s}, 1 \mathrm{H}), 2.68(\mathrm{~m}, 4 \mathrm{H}), 1.22$ $(\mathrm{m}, 6 \mathrm{H}), 0.28(\mathrm{~s}, 9 \mathrm{H}) .{ }^{13} \mathrm{C}$ NMR $\left(100 \mathrm{MHz}, \mathrm{CDCl}_{3}\right) \delta 145.9,143.9,138.8,131.9,122.7,103.3,101.2$, 98.9, 33.6, 27.0, 14.6, 0.1. HRMS calc'd for $\mathrm{C}_{15} \mathrm{H}_{21} \mathrm{ISi}$ : 356.0457 . Found: 356.0452 .

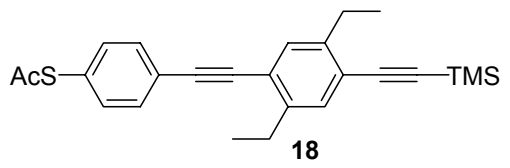

Thioacetic acid S-[4-(2,5-diethyl-4-trimethylsilanylethynyl-phenylethynyl)-phenyl] ester (18). See the general coupling procedure. Used were 17 (2.19 g, $6.15 \mathrm{mmol})$, 1-ethynyl-4-thioacetylbenzene ${ }^{4}$ (1.14 g, $6.47 \mathrm{mmol}), \mathrm{PdCl}_{2}\left(\mathrm{PPh}_{3}\right)_{2}(0.43 \mathrm{~g}, 0.61 \mathrm{mmol}), \mathrm{CuI}(0.23 \mathrm{~g}, 1.21 \mathrm{mmol}), \mathrm{THF}(25 \mathrm{~mL})$, and triethylamine $(21 \mathrm{~mL})$. The reaction was allowed to stir overnight at room temperature. Purification by flash chromatography $\left(3: 1\right.$ Hexanes: $\left.\mathrm{CH}_{2} \mathrm{Cl}_{2}\right)$ afforded the desired product $(1.2 \mathrm{~g}, 48 \%)$ as a yellow oil. IR (KBr) 3413, 2964, 2871, 2349, 2209, 2148, 1902, 1711, 1588, 1493, 1455, 1402, 1350, 1250, $1119,948,834,758,700,613 \mathrm{~cm}^{-1} .{ }^{1} \mathrm{H} \mathrm{NMR}\left(400 \mathrm{MHz}, \mathrm{CDCl}_{3}\right) \delta 7.57(\mathrm{~d}, J=8.4 \mathrm{~Hz} 2 \mathrm{H}), 7.42(\mathrm{~d}, J=$ $8.4 \mathrm{~Hz} 2 \mathrm{H}), 7.37(\mathrm{~d}, J=8.4 \mathrm{~Hz} 2 \mathrm{H}), 2.82(\mathrm{~m}, 4 \mathrm{H}), 2.45(\mathrm{~s}, 3 \mathrm{H}), 1.3(\mathrm{~m}, 6 \mathrm{H}), 0.31(\mathrm{~s}, 9 \mathrm{H}) .{ }^{13} \mathrm{C}$ NMR $\left(100 \mathrm{MHz}, \mathrm{CDCl}_{3}\right) \delta 193.4,144.1,143.6,134.4,132.1,131.7,128.2,124.8,122.8,122.4,103.9,99.5$, 93.4, 90.0, 31.7, 30.4, 27.3, 22.8, 14.8, 0.1. HRMS calc'd for $\mathrm{C}_{25} \mathrm{H}_{28} \mathrm{OSSi}$ : 404.1630. Found: 404.1634. 
Flatt, Yao, Maya, and Tour, Orthogonally Functionalized Oligomers, Supporting Information

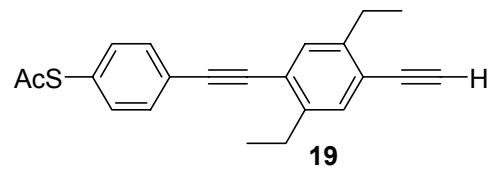

Thioacetic acid S-[4-(2,5-diethyl-4-ethynyl-phenylethynyl)-phenyl] ester (19). 18 (1.0 g, 2.4 mmol) was dissolved in THF $(10 \mathrm{~mL})$, followed by addition of acetic acid $(0.3 \mathrm{~mL}, 4.9 \mathrm{mmol})$, acetic anhydride $(0.3 \mathrm{~mL}, 4.9 \mathrm{mmol})$ and TBAF $(3 \mathrm{~mL}$ of a $1 \mathrm{M}$ solution in THF). The reaction was allowed to stir at room temperature until the starting material was consumed as judged by TLC. The reaction was quenched with water and extracted with EtOAc $(3 \times)$. The combined organic layers were dried over anhydrous $\mathrm{MgSO}_{4}$ and the solvent removed in vacuo. Flash chromatography, silica gel (1:1 hexanes $\left./ \mathrm{CH}_{2} \mathrm{Cl}_{2}\right)$, gave the desired product $(0.8 \mathrm{~g}, 92 \%)$ as a light yellow powder. IR (KBr) 3401, 3291, 3021, 2967, 2931, 2871, 2291, 2213, 2135, 2097, 1906, 1708, 1594, 1493, 1455, 1398, 1351, 1238, 1120, 1012, 950, 897, 829, 756, $613 \mathrm{~cm}^{-1} .{ }^{1} \mathrm{H}$ NMR (400 MHz, $\left.\mathrm{CDCl}_{3}\right) \delta 7.57(\mathrm{~m}, 2 \mathrm{H}), 7.40(\mathrm{~m}$, 4H), $3.36(\mathrm{~s}, 1 \mathrm{H}), 2.82(\mathrm{~m}, 4 \mathrm{H}), 2.43(\mathrm{~s}, 3 \mathrm{H}), 1.28(\mathrm{~m}, 6 \mathrm{H}) .{ }^{13} \mathrm{C}$ NMR $\left(100 \mathrm{MHz}, \mathrm{CDCl}_{3}\right) \delta 193.3$, $144.1,143.5,134.3,132.3,131.6,128.2,124.6,122.6,121.7,93.4,89.7,82.3,60.4,30.3,27.2,27.0$, 14.7, 14.2. HRMS calc'd for $\mathrm{C}_{22} \mathrm{H}_{20} \mathrm{OS}: 332.1235$. Found: 332.1230 .

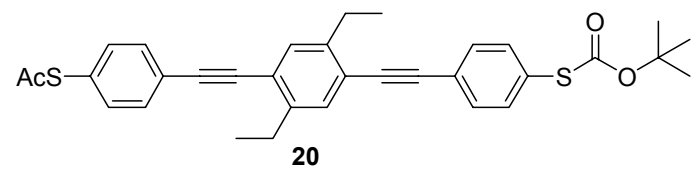

Thioacetic acid S-\{4-[4-(4-tert-butoxycarbonylsulfanyl-phenylethynyl)-2,5-diethylphenylethynyl]-phenyl\} ester (20). See the general coupling procedure. Used were 19 (0.61 g, 1.83 mmol), 7 (0.65 g, $1.93 \mathrm{mmol}), \mathrm{PdCl}_{2}\left(\mathrm{PPh}_{3}\right)_{2}(0.13 \mathrm{~g}, 0.19 \mathrm{mmol}), \mathrm{CuI}(0.07 \mathrm{~g}, 0.37 \mathrm{mmol})$, THF (10 $\mathrm{mL})$, and triethylamine $(1.8 \mathrm{~mL})$. The reaction was allowed to stir overnight at room temperature. Purification by flash chromatography $\left(3: 1\right.$ hexanes $\left./ \mathrm{CH}_{2} \mathrm{Cl}_{2}\right)$ afforded the desired product $(0.63 \mathrm{~g}, 64 \%)$ as a yellow oil. IR (KBr) 3019, 2973, 2932, 2873, 2388, 2205, 1914, 1782, 1716, 1591, 1497, 1456, $1398,1367,1213,1125,1015,951,898,836,768,670,617 \mathrm{~cm}^{-1} .{ }^{1} \mathrm{H} \mathrm{NMR}\left(400 \mathrm{MHz}, \mathrm{CDCl}_{3}\right) \delta 7.57$ $(\mathrm{m}, 6 \mathrm{H}), 7.43(\mathrm{~m}, 4 \mathrm{H}), 2.88(\mathrm{~m}, 4 \mathrm{H}), 2.45(\mathrm{~s}, 3 \mathrm{H}), 1.55(\mathrm{~s}, 9 \mathrm{H}), 1.34(\mathrm{~m}, 6 \mathrm{H}) .{ }^{13} \mathrm{C} \mathrm{NMR}(100 \mathrm{MHz}$, 
Flatt, Yao, Maya, and Tour, Orthogonally Functionalized Oligomers, Supporting Information $\left.\mathrm{CDCl}_{3}\right) \delta 193.5,167.3,143.7,134.7,134.4,132.1,132.0,131.8,129.0,128.2,124.7,124.6,122.5$, 93.6, 90.0, 86.0, 31.7, 30.4, 28.3, 27.3, 22.8, 14.8, 14.3. HRMS calc'd for $\mathrm{C}_{33} \mathrm{H}_{32} \mathrm{O}_{3} \mathrm{~S}_{2}: 540.1793$. Found: 540.1792.

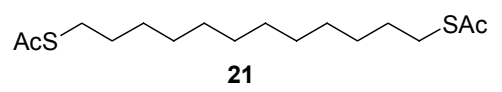

Thioacetic acid $\boldsymbol{S}$-(12-acetylsulfanyl-dodecyl) ester (21). ${ }^{13}$ To a $500 \mathrm{~mL}$ round bottom flask containing a stir bar was added potassium thioacetate $(3.80 \mathrm{~g}, 33.28 \mathrm{mmol})$ and $\mathrm{DMF}^{12}(300 \mathrm{~mL})$. 1,12-Dibromododecane (10.92 g, $33.28 \mathrm{mmol})$ was added in one portion while stirring. The reaction was then allowed to stir at room temperature for $24 \mathrm{~h}$. The mixture was poured into a separatory funnel, washed with water and extracted with $\mathrm{CH}_{2} \mathrm{Cl}_{2}(3 \times)$. The organic layer was dried using anhydrous $\mathrm{MgSO}_{4}$ and the solvent was removed in vacuo. Column chromatography, silica gel (1:1 $\mathrm{CH}_{2} \mathrm{Cl}_{2} /$ hexanes) afforded the product as a white solid $(2.22 \mathrm{~g}, 21 \%)$. Mp: 44-46 ${ }^{\circ} \mathrm{C} .{ }^{1} \mathrm{H}$ NMR (400 $\left.\mathrm{MHz}, \mathrm{CDCl}_{3}\right) \delta 2.85(\mathrm{t}, J=7.28 \mathrm{~Hz}, 4 \mathrm{H}), 2.31(\mathrm{~s}, 6 \mathrm{H}), 1.55$ (quint, $\left.J=7.56,7.28 \mathrm{~Hz}, 4 \mathrm{H}\right), 1.35-1.20$ $(\mathrm{m}, 16 \mathrm{H})$.

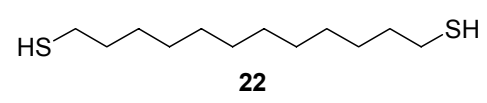

Dodecane-1,12-dithiol (22). ${ }^{14}$ To a $250 \mathrm{~mL}$ round bottom flask containing a stir bar was added 21 $(1.00 \mathrm{~g}, 3.14 \mathrm{mmol})$ and $60 \mathrm{~mL}$ of degassed $3 \mathrm{M} \mathrm{NaOH}$ in acetone. The reaction was allowed to stir at room temperature for $4 \mathrm{~h}$ and neutralized to $\mathrm{pH} 7$ with $1 \mathrm{M} \mathrm{HCl}$. The organics were extracted with $\mathrm{CH}_{2} \mathrm{Cl}_{2}(3 \times)$, dried using anhydrous $\mathrm{MgSO}_{4}$, and removed in vacuo. Column chromatography, silica gel $\left(\mathrm{CH}_{2} \mathrm{Cl}_{2}\right)$ afforded the product as a white solid $(0.41 \mathrm{~g}, 56 \%)$. Mp: $31-32{ }^{\circ} \mathrm{C}$. FTIR (KBr) 2952 , 2917, 2850, 1471, 1280, 1231, 717, $707 \mathrm{~cm}^{-1} .{ }^{1} \mathrm{H}$ NMR $\left(400 \mathrm{MHz}, \mathrm{CDCl}_{3}\right) \delta 2.52$ (quart, $J=7.5 \mathrm{~Hz}$, 4H), 1.59 (quint, $J=7.6 \mathrm{~Hz}, 4 \mathrm{H}), 1.39-1.24(\mathrm{~m}, 18 \mathrm{H}) .{ }^{13} \mathrm{C} \mathrm{NMR}\left(100 \mathrm{MHz}, \mathrm{CDCl}_{3}\right) \delta 34.5,30.0,29.9$. 29.5, 28.8, 25.1. HRMS calc'd for $\mathrm{C}_{12} \mathrm{H}_{26} \mathrm{~S}_{2}$ : 234.1476. Found: 234.1476. 
Flatt, Yao, Maya, and Tour, Orthogonally Functionalized Oligomers, Supporting Information

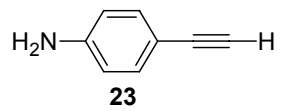

1-Amino-4-ethynylbenzene (23). ${ }^{15}$ See the general coupling procedure. Used were 4-iodoaniline (8.76 g, $40.00 \mathrm{mmol})$, trimethylsilylacetylene (6.8 mL, $48.12 \mathrm{mmol}), \mathrm{PdCl}_{2}\left(\mathrm{PPh}_{3}\right)_{2}(1.40 \mathrm{~g}, 1.99 \mathrm{mmol})$, CuI (0.76 g, $3.99 \mathrm{mmol})$, THF (50 mL), and $i$ - $\operatorname{Pr}_{2}$ NEt $(28 \mathrm{~mL})$. Flash chromatography, silica gel (4:1 hexane/EtOAc) gave a brown yellow solid. The solid $(6.10 \mathrm{~g}, 32.22 \mathrm{mmol})$ was dissolved in $\mathrm{MeOH}$ $(60 \mathrm{~mL})$ and $\mathrm{K}_{2} \mathrm{CO}_{3}(13.40 \mathrm{~g}, 96.95 \mathrm{mmol})$ was added. The reaction was stirred for $8 \mathrm{~h}$. The mixture was poured into water and extracted with EtOAc $(2 \times)$. The extracts were washed with water $(1 \times)$ and brine $(1 \times)$. The organics were dried using anhydrous $\mathrm{MgSO}_{4}$ and concentrated in vacuo, the brown oil residue was filtered through a short silica gel plug $\left(\mathrm{CH}_{2} \mathrm{Cl}_{2}\right)$ to give 23 as a brown solid (3.98 g, 85\%). ${ }^{1} \mathrm{H}$ NMR $\left(400 \mathrm{MHz}, \mathrm{CDCl}_{3}\right) \delta 7.32(\mathrm{~d}, J=8.8 \mathrm{~Hz}, 2 \mathrm{H}), 6.62(\mathrm{~d}, J=8.8 \mathrm{~Hz}, 2 \mathrm{H}), 3.84(\mathrm{~s}, 2 \mathrm{H}), 2.99$ (s, $1 \mathrm{H})$.

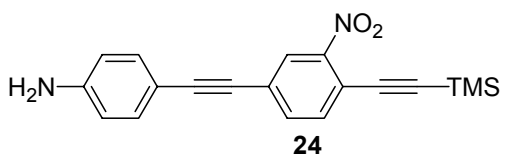

4-(3-Nitro-4-trimethylsilanylethynyl-phenylethynyl)-phenylamine (24). See the general coupling procedure. Used were 23 (0.86 g, $7.34 \mathrm{mmol}), 9$ (2.65 g, $8.89 \mathrm{mmol}), \mathrm{Pd}(\mathrm{dba})_{2}(0.19 \mathrm{~g}, 0.34 \mathrm{mmol})$, $\mathrm{PPh}_{3}(0.36 \mathrm{~g}, 1.36 \mathrm{mmol}), \mathrm{CuI}(0.13 \mathrm{~g}, 0.67 \mathrm{mmol})$, THF $(15 \mathrm{~mL})$ and $i$-Pr $2 \mathrm{NEt}(3.5 \mathrm{~mL})$. Flash chromatography, silica gel (3:1 hexane/EtOAc) gave 24 as red solid (1.53 g, 62\%). Mp: $121-123{ }^{\circ} \mathrm{C}$. FTIR (KBr) 3477, 3383, 2198, 2150, 1630, 1599, 1537, 1519, 1347, 1323, 1298, 1249, 1221, 1177, 1156, 1134, 852, 825, 758, 705, 660, 529, $483 \mathrm{~cm}^{-1} .{ }^{1} \mathrm{H}$ NMR $\left(400 \mathrm{MHz},\left(\mathrm{CD}_{3}\right)_{2} \mathrm{CO}\right) \delta 8.08(\mathrm{~d}, J=1.5$ $\mathrm{Hz}, 1 \mathrm{H}), 7.74(\mathrm{dd}, J=8.1,1.5 \mathrm{~Hz}, 1 \mathrm{H}), 7.70(\mathrm{~d}, J=8.1 \mathrm{~Hz}, 1 \mathrm{H}), 7.31(\mathrm{~d}, J=8.6 \mathrm{~Hz}, 2 \mathrm{H}), 6.70(\mathrm{~d}, J=$ $8.6 \mathrm{~Hz}, 2 \mathrm{H}), 5.22(\mathrm{~s}, 2 \mathrm{H}), 0.27(\mathrm{~s}, 9 \mathrm{H}) .{ }^{13} \mathrm{C} \mathrm{NMR}\left(100 \mathrm{MHz},\left(\mathrm{CD}_{3}\right)_{2} \mathrm{CO}\right) \delta 151.5,151.1,135.9,135.6$, 134.2, 127.1, 126.7, 116.7, 114.9, 109.6, 104.8, 100.2, 96.9, 85.5, -0.3. HRMS calc'd for $\mathrm{C}_{19} \mathrm{H}_{18} \mathrm{~N}_{2} \mathrm{O}_{2} \mathrm{Si}: 334.1138$. Found: 334.1131. 
Flatt, Yao, Maya, and Tour, Orthogonally Functionalized Oligomers, Supporting Information

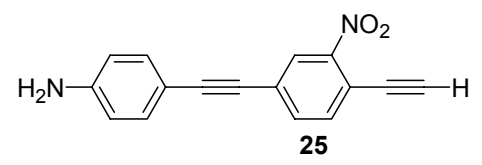

4-(4-Ethynyl-3-nitro-phenylethynyl)-phenylamine (25). To a solution of 24 (1.53 g, $4.57 \mathrm{mmol})$ in THF $(10 \mathrm{~mL})$ and $\mathrm{MeOH}(25 \mathrm{~mL})$ was added $\mathrm{K}_{2} \mathrm{CO}_{3}(1.90 \mathrm{~g}, 13.75 \mathrm{mmol})$. The mixture was stirred for $3 \mathrm{~h}$ and poured into THF $(50 \mathrm{~mL})$ and EtOAc $(50 \mathrm{~mL})$. The solution was washed with water, brine $(2 \times)$, and dried over anhydrous $\mathrm{MgSO}_{4}$. Removal of solvent followed by flash chromatography, silica gel (3:2 hexane/EtOAc) gave 25 as red solid (0.97 g, 80\%). Mp: 173-175 ${ }^{\circ} \mathrm{C}$. FTIR (KBr) 3480, 3006, 2970, 1716, 1423, 1365, 1224, $530 \mathrm{~cm}^{-1} .{ }^{1} \mathrm{H}$ NMR $\left(400 \mathrm{MHz},\left(\mathrm{CD}_{3}\right)_{2} \mathrm{CO}\right) \delta 8.09(\mathrm{t}, J=1.1 \mathrm{~Hz}, 1 \mathrm{H})$, $7.76(\mathrm{~d}, J=1.1 \mathrm{~Hz}, 2 \mathrm{H}), 7.31(\mathrm{~d}, J=8.7 \mathrm{~Hz}, 2 \mathrm{H}), 6.70(\mathrm{~d}, J=8.7 \mathrm{~Hz}, 2 \mathrm{H}), 5.23(\mathrm{~s}, 2 \mathrm{H}), 4.26(\mathrm{~s}, 1 \mathrm{H})$. ${ }^{13} \mathrm{C}$ NMR $\left(100 \mathrm{MHz},\left(\mathrm{CD}_{3}\right){ }_{2} \mathrm{CO}\right) \delta 151.0,136.2,135.6,134.1,127.1,126.9,116.0,114.8,109.5,96.8$, 88.0, 85.3, 78.9. HRMS calc'd for $\mathrm{C}_{16} \mathrm{H}_{10} \mathrm{~N}_{2} \mathrm{O}_{2}$ : 262.0742. Found: 262.0737.

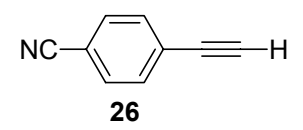

4-Ethynylbenzonitrile (26). ${ }^{16}$ See the general coupling procedure. Used were 4-bromobenzonitrile (5.46 g, $30.00 \mathrm{mmol})$, trimethylsilylacetylene (5.1 mL, $36.09 \mathrm{mmol}), \mathrm{Pd}(\mathrm{dba})_{2}(0.86 \mathrm{~g}, 1.54 \mathrm{mmol})$, $\mathrm{PPh}_{3}(1.57 \mathrm{~g}, 5.93 \mathrm{mmol}), \mathrm{CuI}(0.57 \mathrm{~g}, 2.99 \mathrm{mmol}), i$-Pr $2 \mathrm{NEt}(21 \mathrm{~mL})$ and THF $(40 \mathrm{~mL})$. The reaction was stirred at $55^{\circ} \mathrm{C}$ for $4.5 \mathrm{~h}$. The mixture was diluted with hexane and washed with water $(2 \times)$ and then dried over anhydrous $\mathrm{MgSO}_{4}$. The solvent was removed in vacuo and the residue was redissolved in $\mathrm{CH}_{2} \mathrm{Cl}_{2}(20 \mathrm{~mL})$. The solution was diluted with hexane $(150 \mathrm{~mL})$ and concentrated to small volume. The precipitates were filtered. The filtrate was concentrated and filtered through a silica gel plug (4:1 hexane $/ \mathrm{CH}_{2} \mathrm{Cl}_{2}$ then $2: 1$ hexane $/ \mathrm{CH}_{2} \mathrm{Cl}_{2}$ ) to give 4-(2'-trimethylsilylethynyl)benzonitrile as white solid. The solid was dissolved in $\mathrm{MeOH}(100 \mathrm{~mL})$ and $\mathrm{K}_{2} \mathrm{CO}_{3}(3.80 \mathrm{~g}, 27.49 \mathrm{mmol})$ was added. The mixture was stirred for $3 \mathrm{~h}$ and then diluted with EtOAc. The organic solution was washed water $(2 \times)$ and dried over anhydrous $\mathrm{MgSO}_{4}$. The solvent was removed in vacuo and the residue was purified by 
Flatt, Yao, Maya, and Tour, Orthogonally Functionalized Oligomers, Supporting Information flash chromatography through a short silica gel plug $\left(1: 1\right.$ hexane $\left./ \mathrm{CH}_{2} \mathrm{Cl}_{2}\right)$. The collected fractions were concentrated to nearly dry and then diluted with hexane. The solid was filtered to give $\mathbf{2 6}(2.30 \mathrm{~g}, 60 \%$ over two steps) as yellow crystals. ${ }^{1} \mathrm{H} \mathrm{NMR}\left(400 \mathrm{MHz}, \mathrm{CDCl}_{3}\right) \delta 7.65(\mathrm{~d}, J=8.4 \mathrm{~Hz}, 2 \mathrm{H}), 7.60(\mathrm{~d}, J=$ $8.6 \mathrm{~Hz}, 2 \mathrm{H}), 3.33(\mathrm{~s}, 1 \mathrm{H})$.

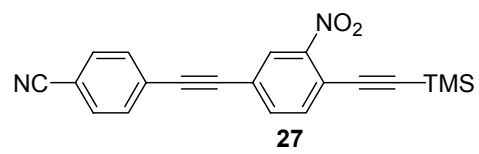

4-(3-Nitro-4-trimethylsilanylethynyl-phenylethynyl)-benzonitrile (27). See the general coupling procedure. Used were $26(0.72 \mathrm{~g}, 5.66 \mathrm{mmol}), 9(1.99 \mathrm{~g}, 6.88 \mathrm{mmol}), \mathrm{Pd}(\mathrm{dba})_{2}(0.16 \mathrm{~g}, 0.28 \mathrm{mmol})$, $\mathrm{PPh}_{3}(0.29 \mathrm{~g}, 1.12 \mathrm{mmol}), \mathrm{CuI}(0.11 \mathrm{~g}, 0.56 \mathrm{mmol})$, THF (10 mL) and $i$-Pr $2 \mathrm{NEt}(3.9 \mathrm{~mL})$. Purification through a short silica gel plug (hexane, then 2:1 hexane $\left./ \mathrm{CH}_{2} \mathrm{Cl}_{2}\right)$ gave 27 as a yellow solid (1.14 g, 59\%). Mp: $129-131^{\circ} \mathrm{C}$. FTIR (KBr) 3044, 2951, 2896, 2227, 2159, 1604, 1528, 1363, 1247, 865, 849, 766, 647, $556 \mathrm{~cm}^{-1} .{ }^{1} \mathrm{H} \mathrm{NMR}\left(400 \mathrm{MHz}, \mathrm{CDCl}_{3}\right) \delta 8.18(\mathrm{dd}, J=1.0,0.5 \mathrm{~Hz}, 1 \mathrm{H}), 7.70-7.66(\mathrm{~m}, 4 \mathrm{H})$, $7.63(\mathrm{~d}, J=8.7 \mathrm{~Hz}, 2 \mathrm{H}) .{ }^{13} \mathrm{C} \mathrm{NMR}\left(100 \mathrm{MHz}, \mathrm{CDCl}_{3}\right) \delta 150.2,135.38,135.36,132.5,132.4,127.7$, 127.0, 123.4, 118.7, 118.4, 112.7, 106.8, 99.1, 91.6, 90.8, -0.3. HRMS calc'd for $\mathrm{C}_{20} \mathrm{H}_{16} \mathrm{~N}_{2} \mathrm{O}_{2} \mathrm{Si}_{\text {: }}$ 344.0981. Found: 344.0978.

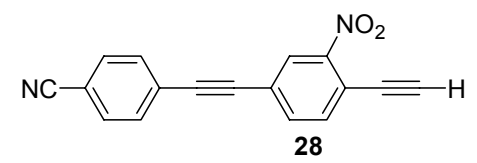

4-(4-Ethynyl-3-nitro-phenylethynyl)-benzonitrile (28). 27 (0.85 g, 2.5 mmol) was dissolved in THF $(20 \mathrm{~mL})$. Acetic acid $(0.3 \mathrm{~mL}, 5 \mathrm{mmol})$ was added, followed by TBAF ( $3 \mathrm{~mL}$ of a $1.0 \mathrm{M}$ solution in THF). After addition, TLC indicated the starting materials were completely consumed. The mixture was diluted with EtOAc and washed with water $(2 \times)$, brine $(1 \times)$ and dried over anhydrous $\mathrm{MgSO}_{4}$. Purification through a short silica gel plug $\left(1: 1\right.$ hexane $\left./ \mathrm{CH}_{2} \mathrm{Cl}_{2}\right)$ and concentration of the collected fractions to about $50 \mathrm{~mL}$ followed by filtration gave 28 as green solid $(0.38 \mathrm{~g}, 57 \%)$. Mp: $179-182{ }^{\circ} \mathrm{C}$. 
Flatt, Yao, Maya, and Tour, Orthogonally Functionalized Oligomers, Supporting Information FTIR (KBr) 3280, 3082, 2360, 2219, 1601, 1538, 1520, 1355, 838, 682, 654, $553 \mathrm{~cm}^{-1}$. ${ }^{1} \mathrm{H}$ NMR (400 MHz, DMSO) $\delta 8.32(\mathrm{~d}, J=1.6 \mathrm{~Hz}, 1 \mathrm{H}), 7.95-7.92(\mathrm{~m}, 3 \mathrm{H}), 7.84(\mathrm{~d}, J=8.1 \mathrm{~Hz}, 1 \mathrm{H}), 7.81(\mathrm{~d}, J=8.5$ $\mathrm{Hz}, 2 \mathrm{H}), 4.93(\mathrm{~s}, 1 \mathrm{H}) .{ }^{13} \mathrm{C}$ NMR (100 MHz, DMSO) $\delta 150.2,135.8,135.5,132.7,132.4,127.4,126.1$, 123.0, 118.3, 116.5, 111.8, 91.5, 90.5, 90.3, 77.9. HRMS calc'd for $\mathrm{C}_{17} \mathrm{H}_{8} \mathrm{~N}_{2} \mathrm{O}_{2}$ : 272.0586. Found: 272.0585 .

\section{${ }^{1} \mathrm{H}$ and ${ }^{13} \mathrm{C}$ NMR Spectra}


Flatt, Yao, Maya, and Tour, Orthogonally Functionalized Oligomers, Supporting Information

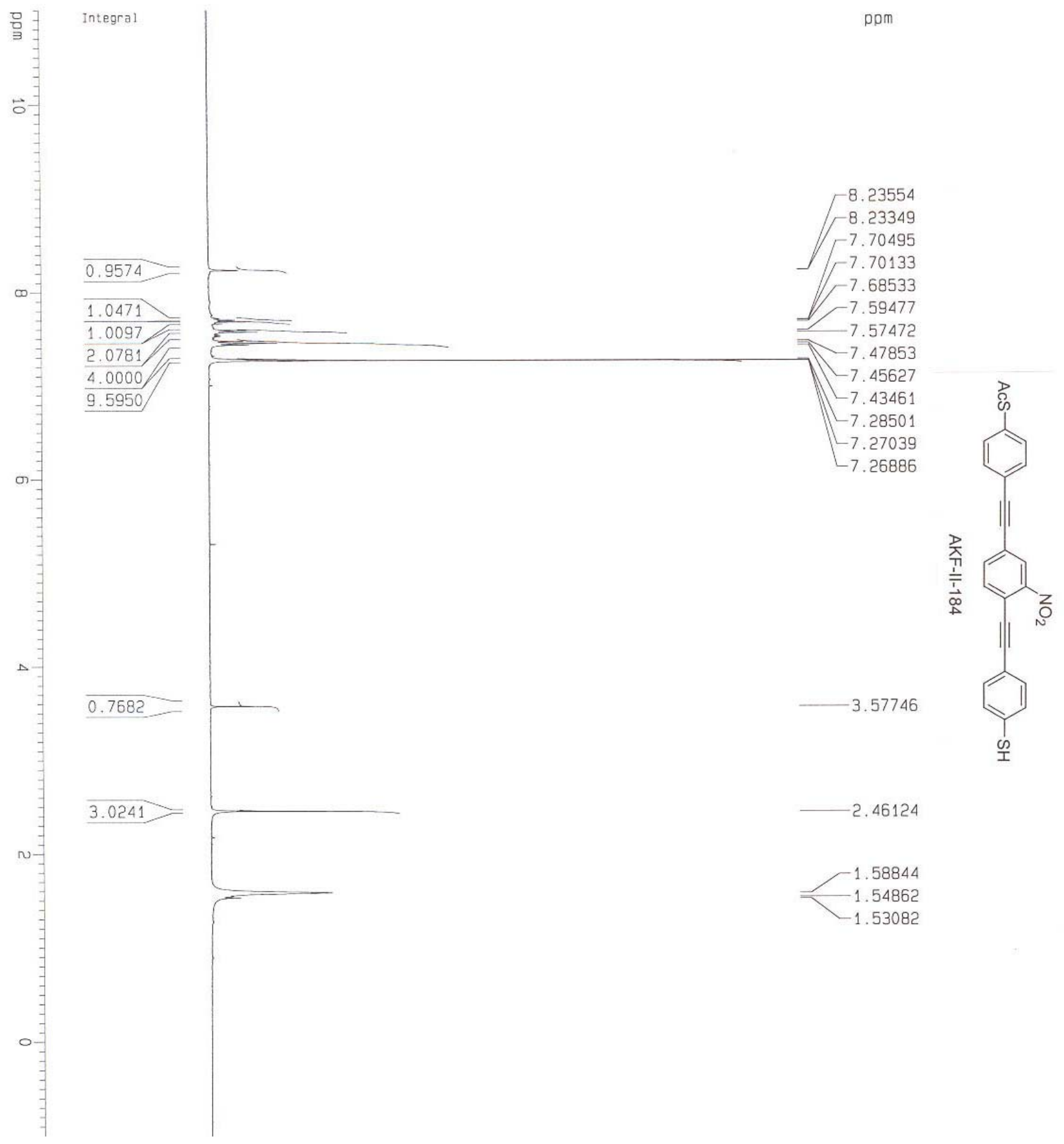

Figure S1: ${ }^{1} \mathrm{H}$ NMR of 1. 
Flatt, Yao, Maya, and Tour, Orthogonally Functionalized Oligomers, Supporting Information

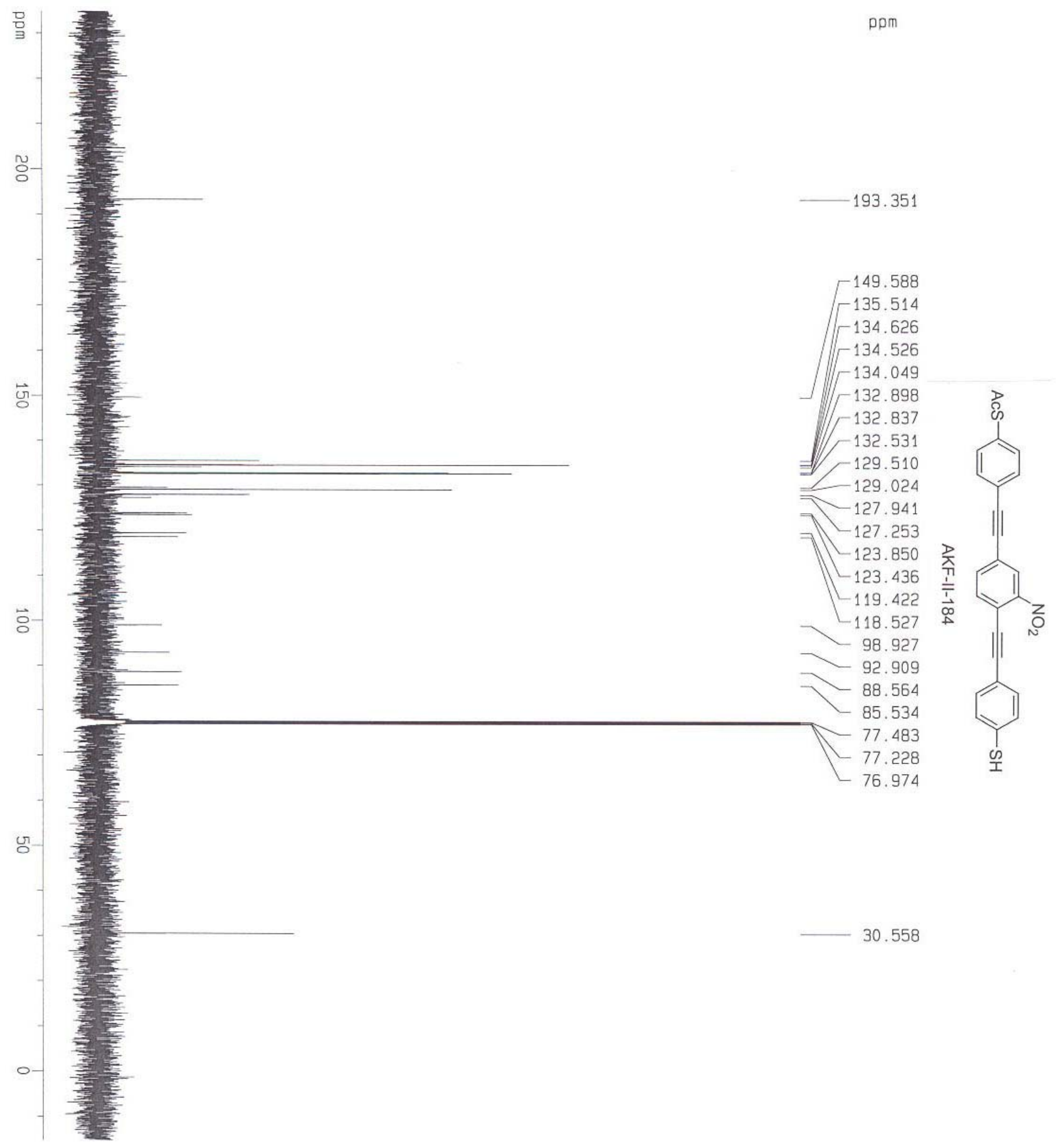

Figure S2: ${ }^{13} \mathrm{C}$ NMR of 1 . 
Flatt, Yao, Maya, and Tour, Orthogonally Functionalized Oligomers, Supporting Information

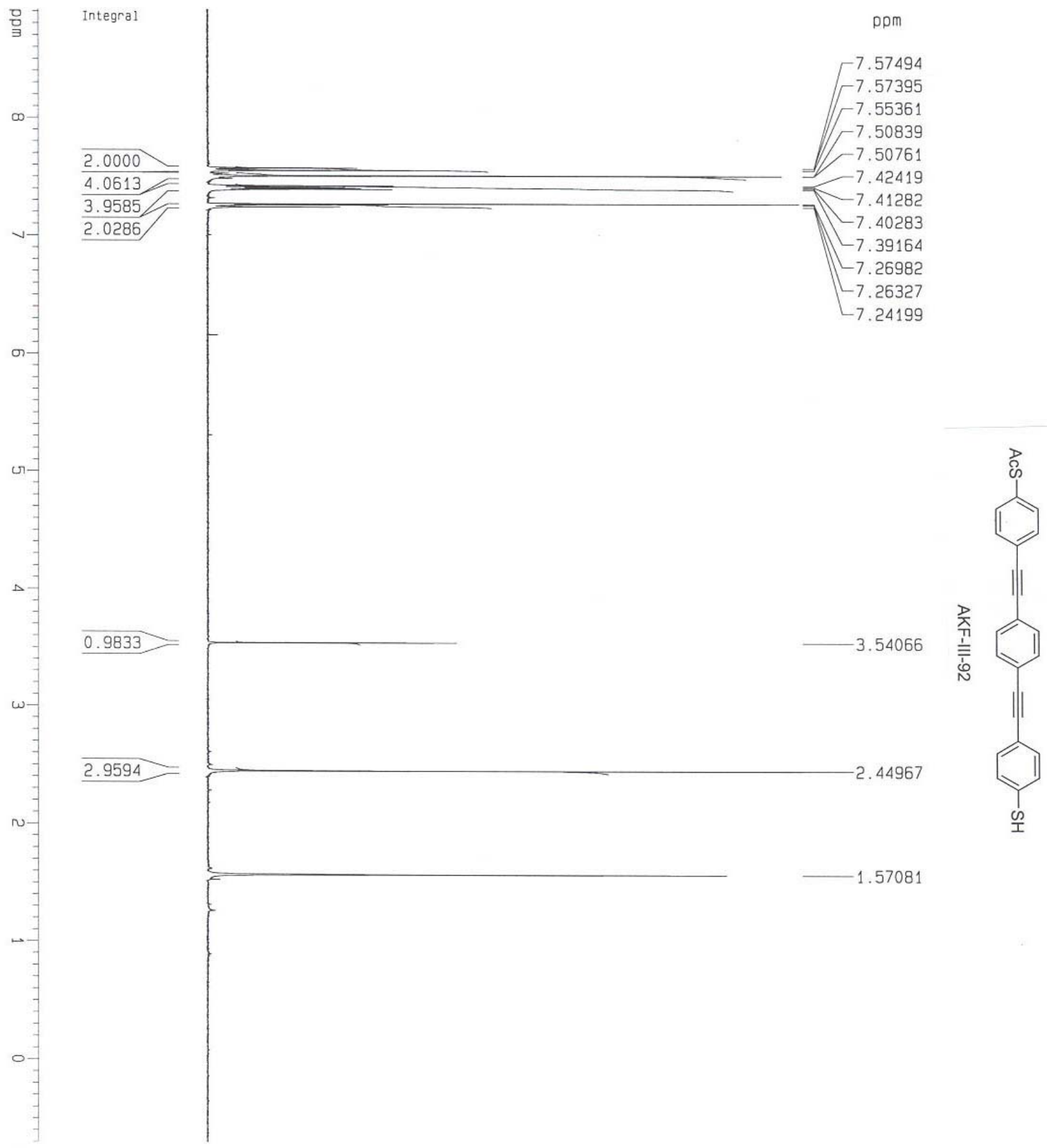

Figure S3: ${ }^{1} \mathrm{H}$ NMR of 2. 
Flatt, Yao, Maya, and Tour, Orthogonally Functionalized Oligomers, Supporting Information

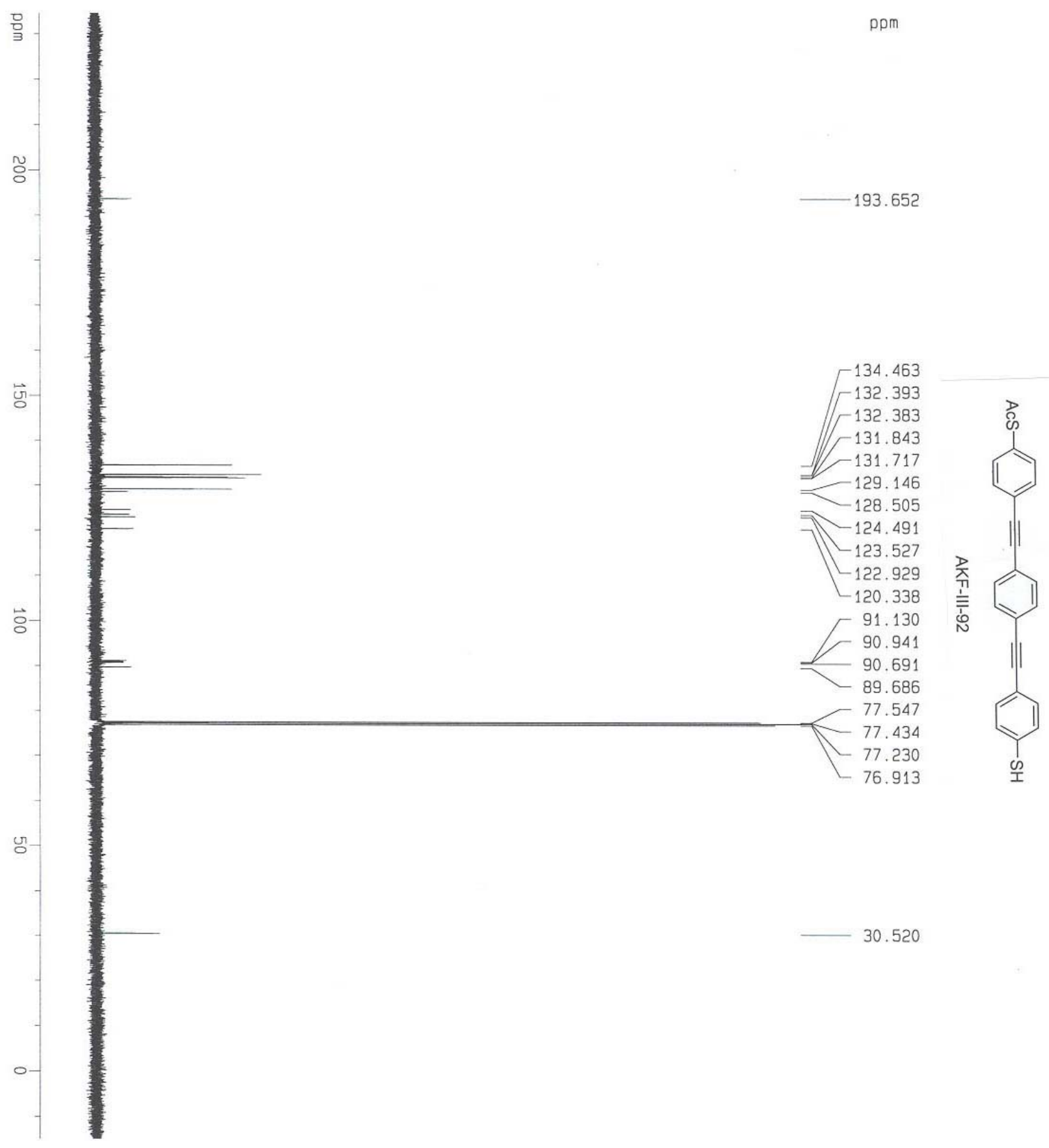

Figure S4: ${ }^{13} \mathrm{C}$ NMR of 2 . 
Flatt, Yao, Maya, and Tour, Orthogonally Functionalized Oligomers, Supporting Information

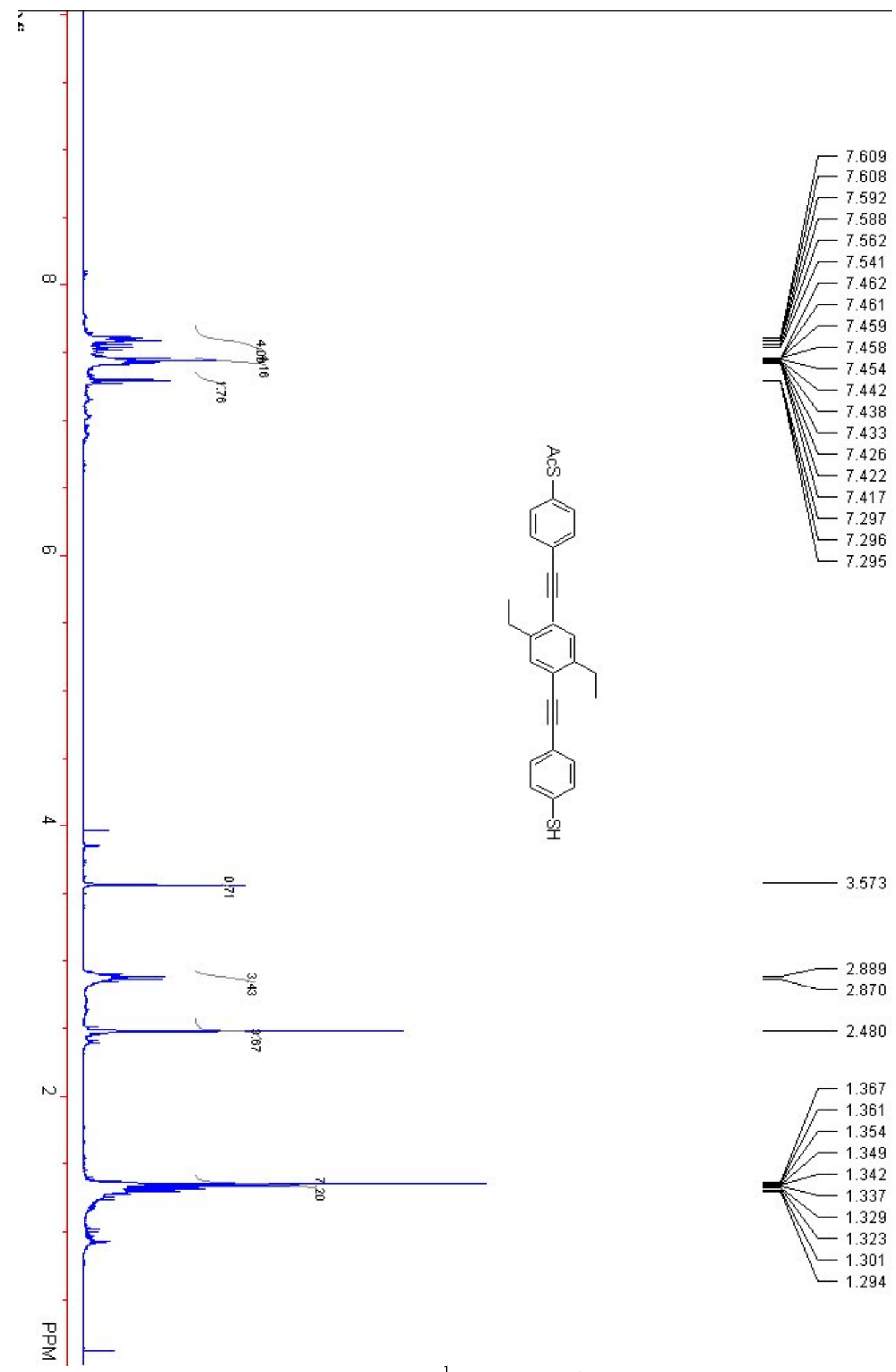

Figure S5: ${ }^{1} \mathrm{H}$ NMR of 3. 
Flatt, Yao, Maya, and Tour, Orthogonally Functionalized Oligomers, Supporting Information
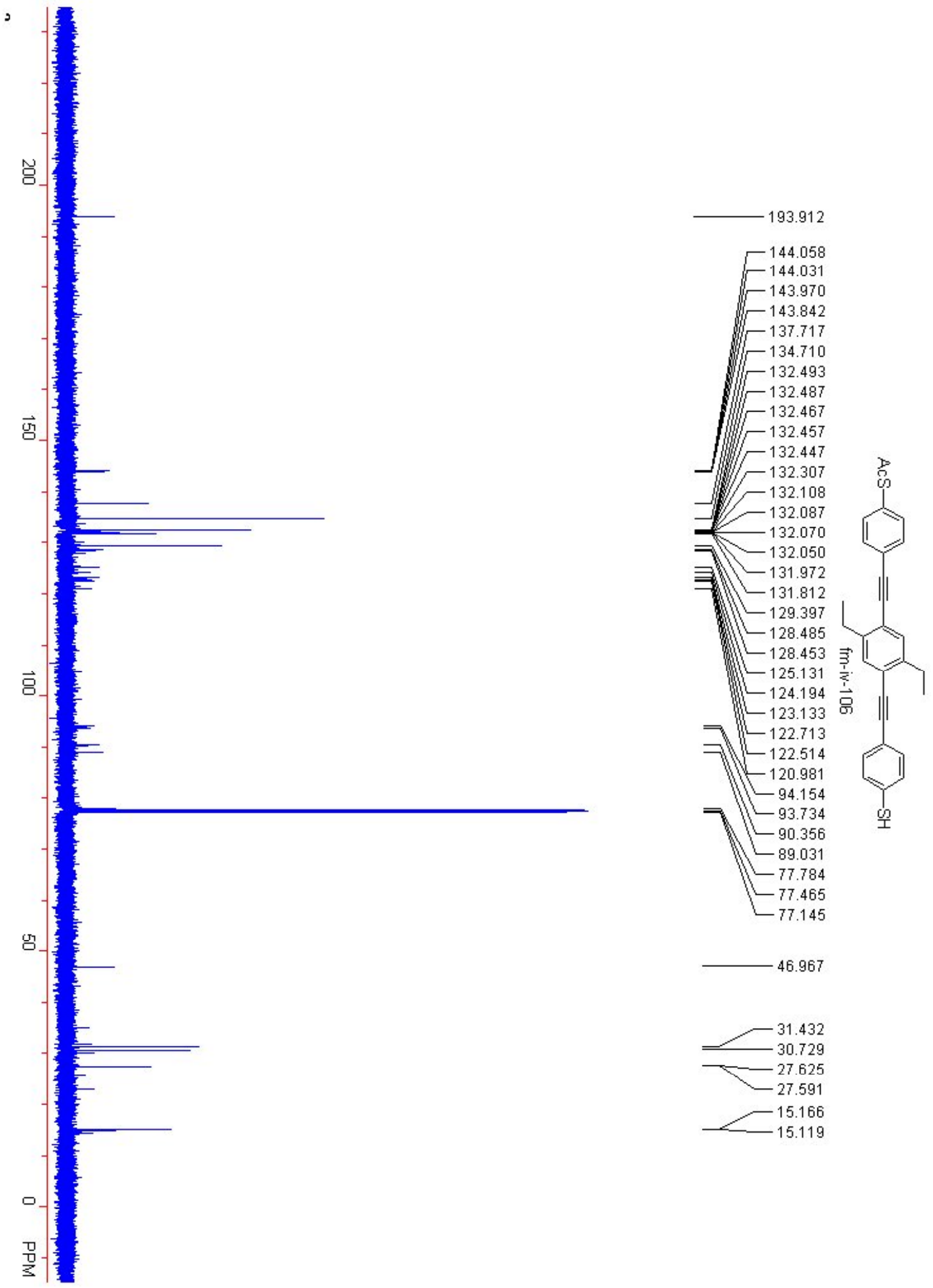

Figure S6: ${ }^{13} \mathrm{C}$ NMR of 3 . 
Flatt, Yao, Maya, and Tour, Orthogonally Functionalized Oligomers, Supporting Information

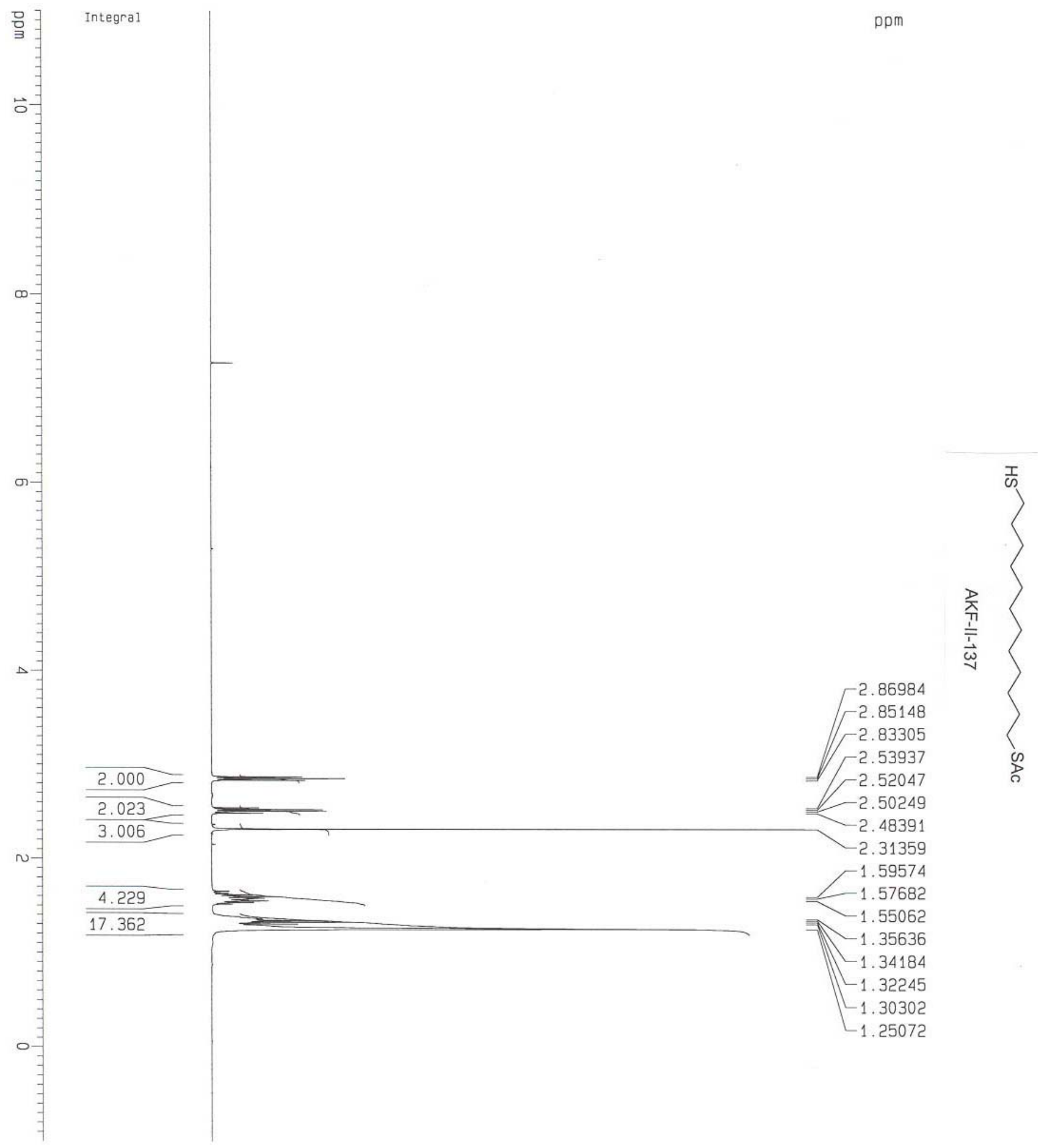

Figure S7: ${ }^{1} \mathrm{H}$ NMR of 4. 
Flatt, Yao, Maya, and Tour, Orthogonally Functionalized Oligomers, Supporting Information

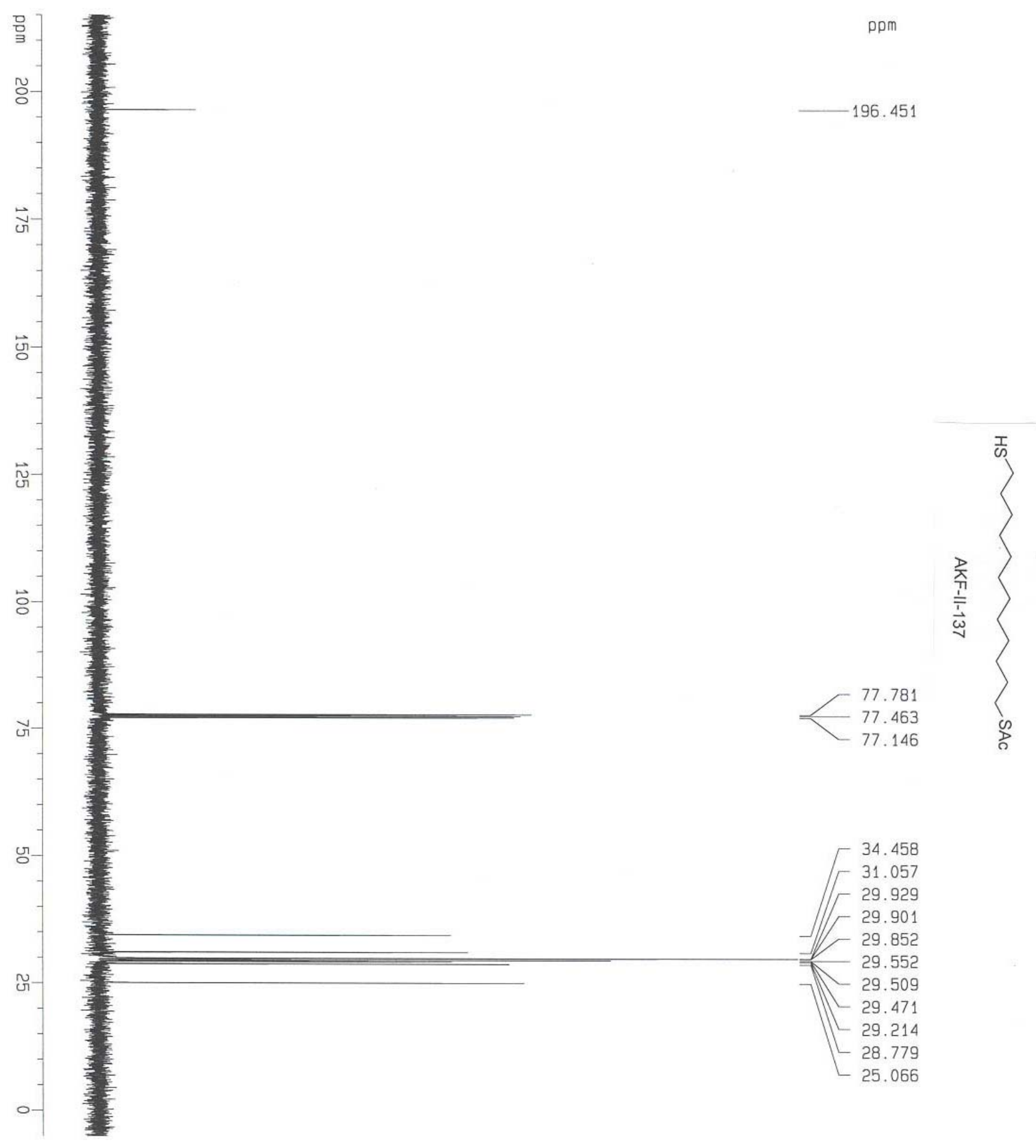

Figure S8: ${ }^{13} \mathrm{C}$ NMR of 4 . 
Flatt, Yao, Maya, and Tour, Orthogonally Functionalized Oligomers, Supporting Information

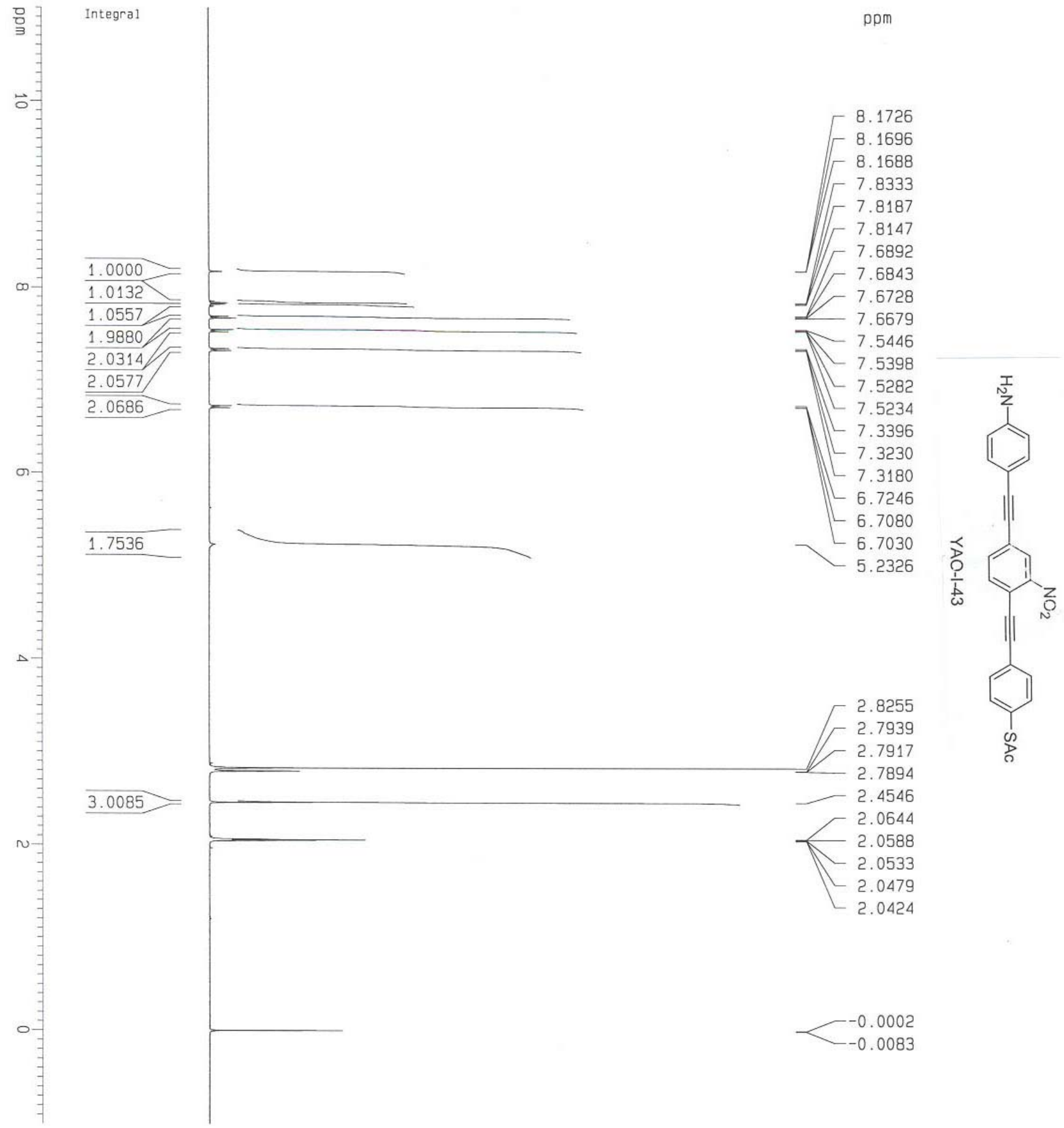

Figure S9: ${ }^{1} \mathrm{H}$ NMR of 5. 
Flatt, Yao, Maya, and Tour, Orthogonally Functionalized Oligomers, Supporting Information

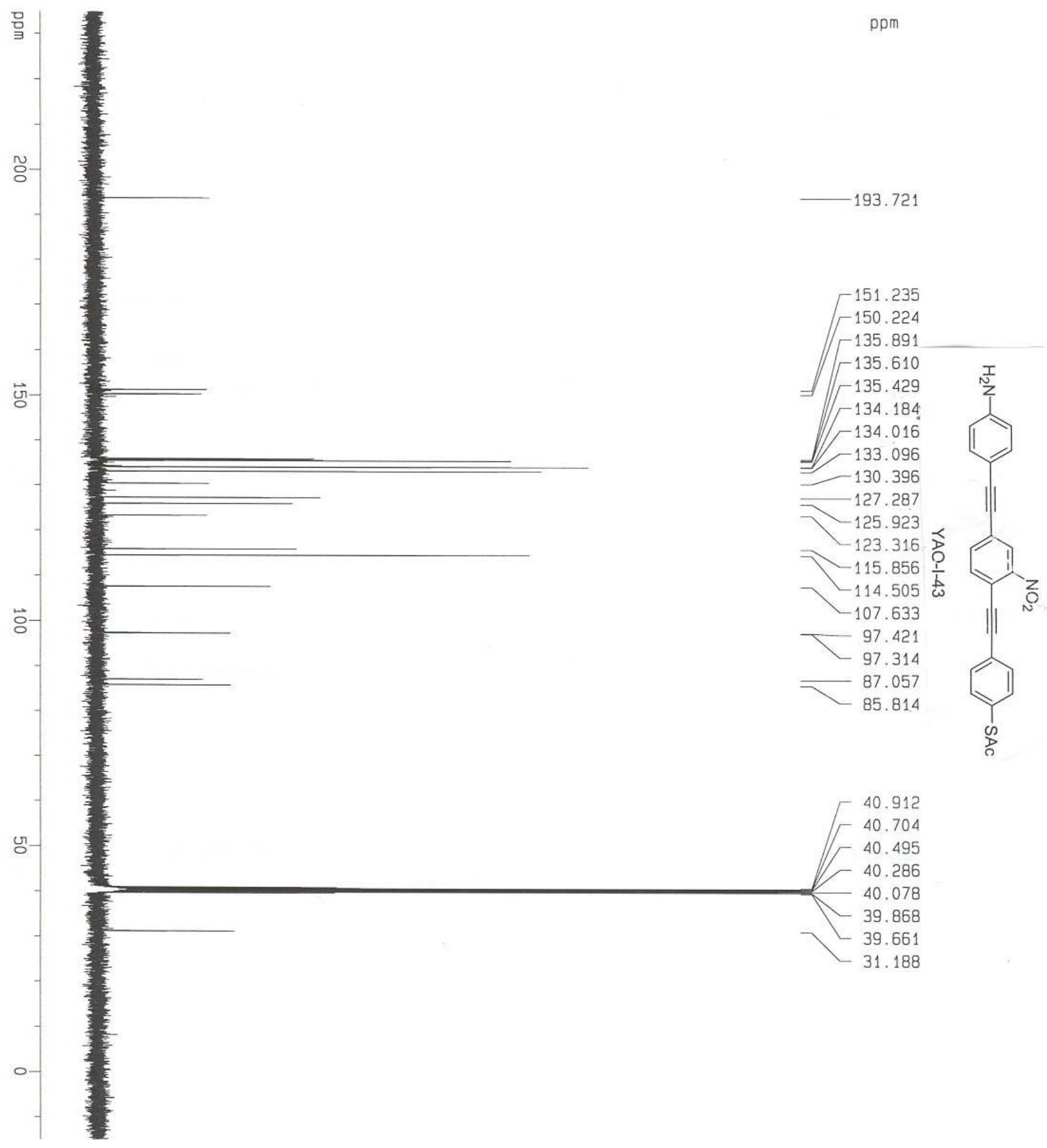

Figure S10: ${ }^{13} \mathrm{C}$ NMR of 5 . 
Flatt, Yao, Maya, and Tour, Orthogonally Functionalized Oligomers, Supporting Information

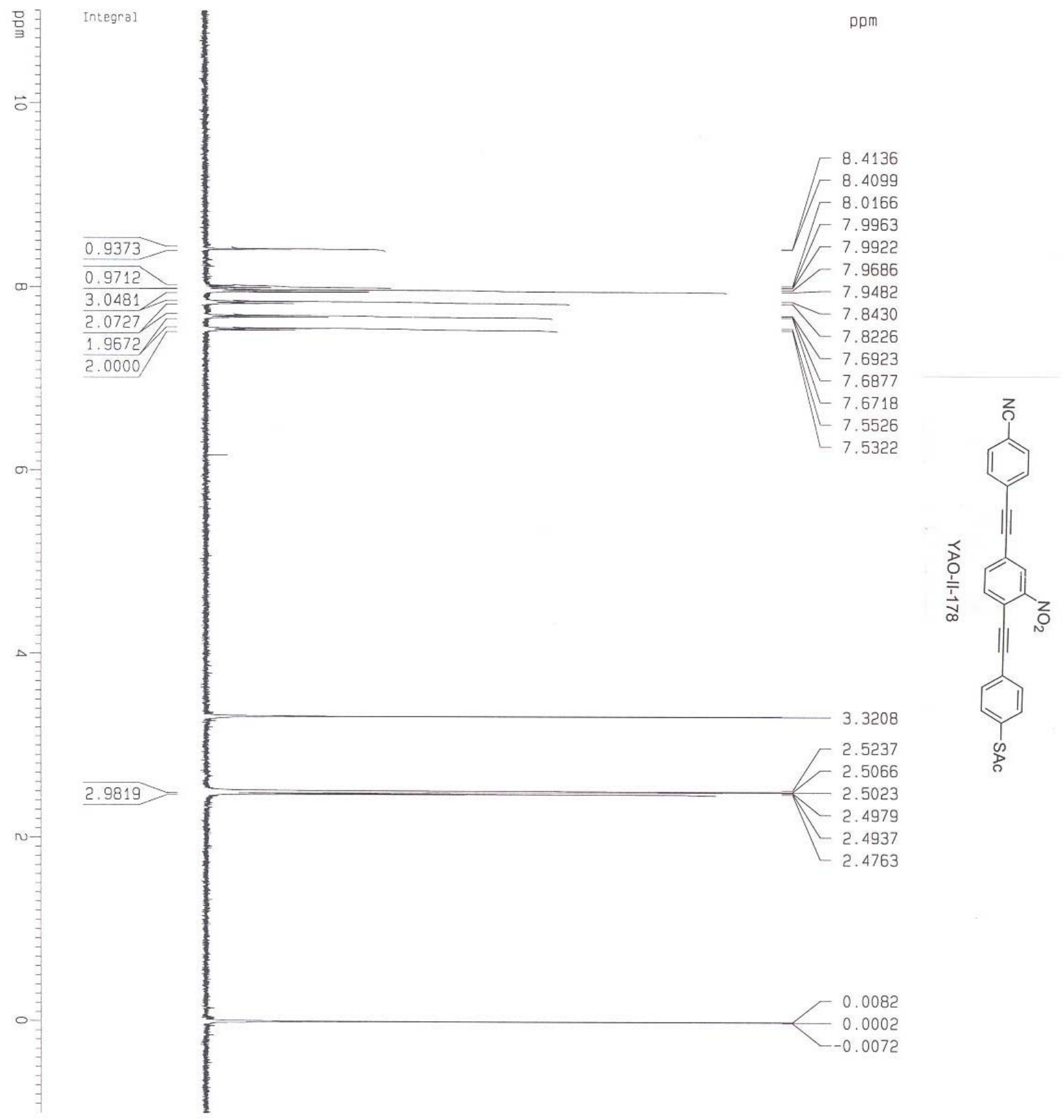

Figure S11: ${ }^{1} \mathrm{H}$ NMR of 6. 
Flatt, Yao, Maya, and Tour, Orthogonally Functionalized Oligomers, Supporting Information

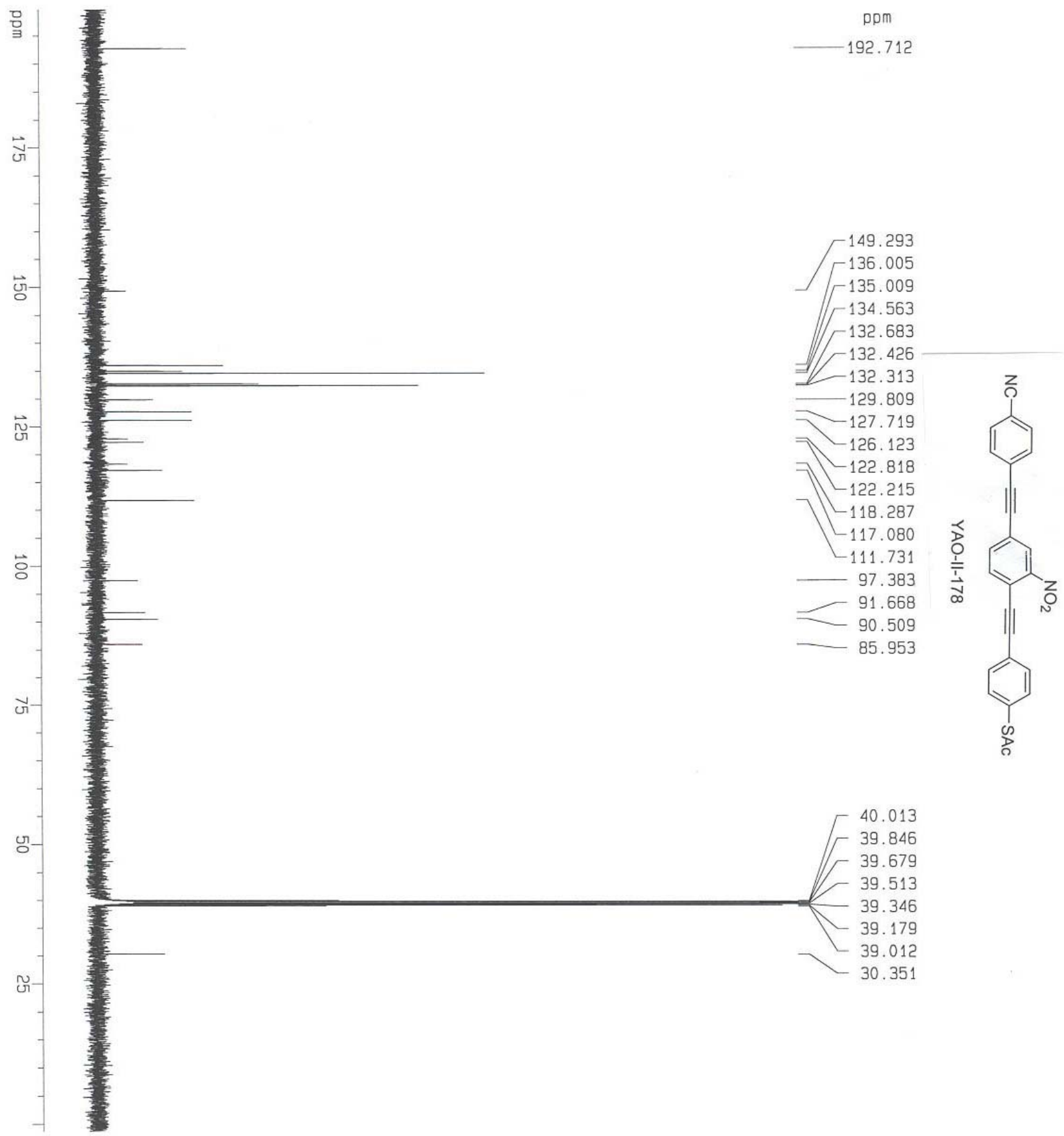

Figure S12: ${ }^{13} \mathrm{C}$ NMR of 6 . 
Flatt, Yao, Maya, and Tour, Orthogonally Functionalized Oligomers, Supporting Information

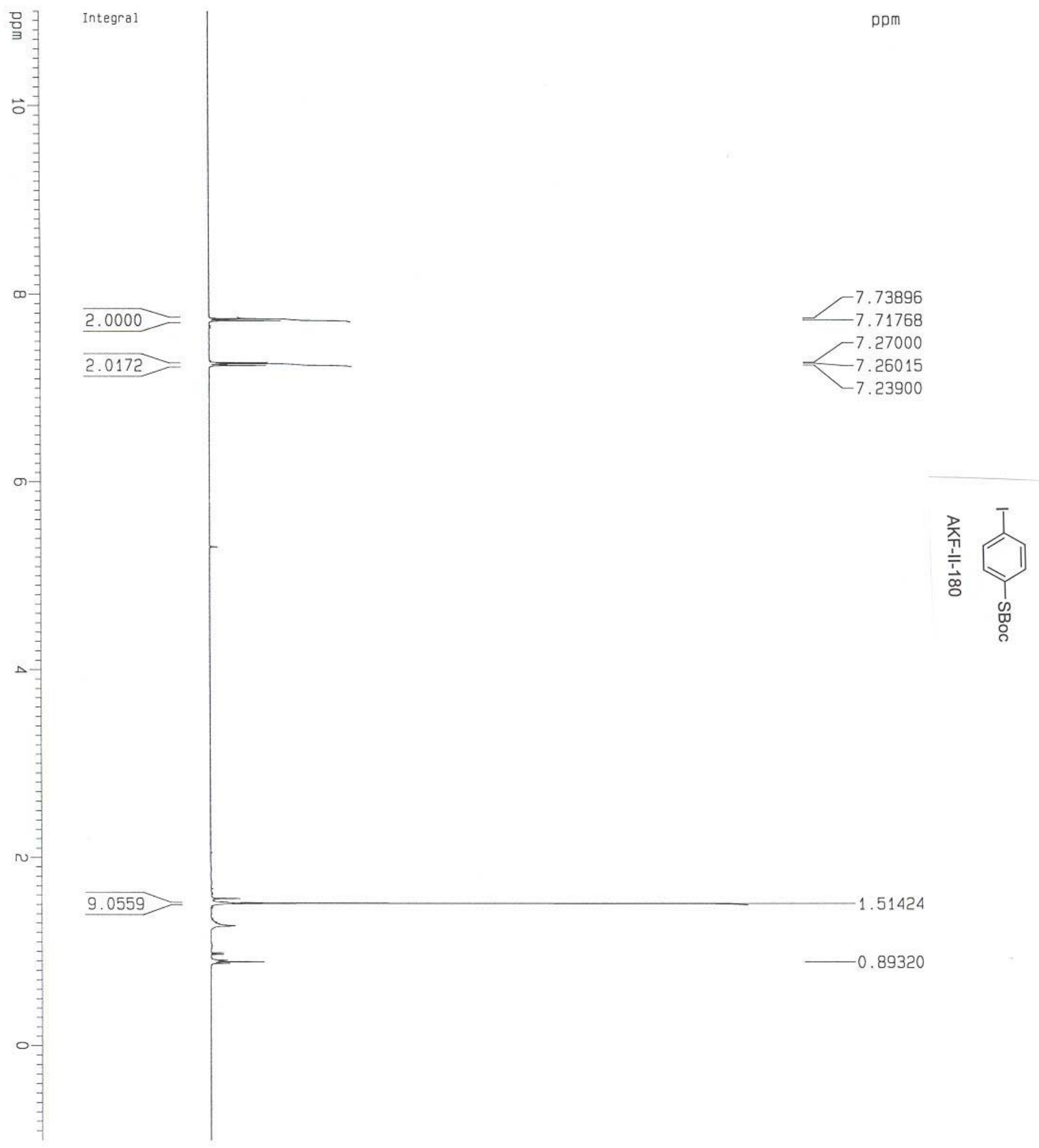

Figure S13: ${ }^{1} \mathrm{H}$ NMR of 7. 
Flatt, Yao, Maya, and Tour, Orthogonally Functionalized Oligomers, Supporting Information

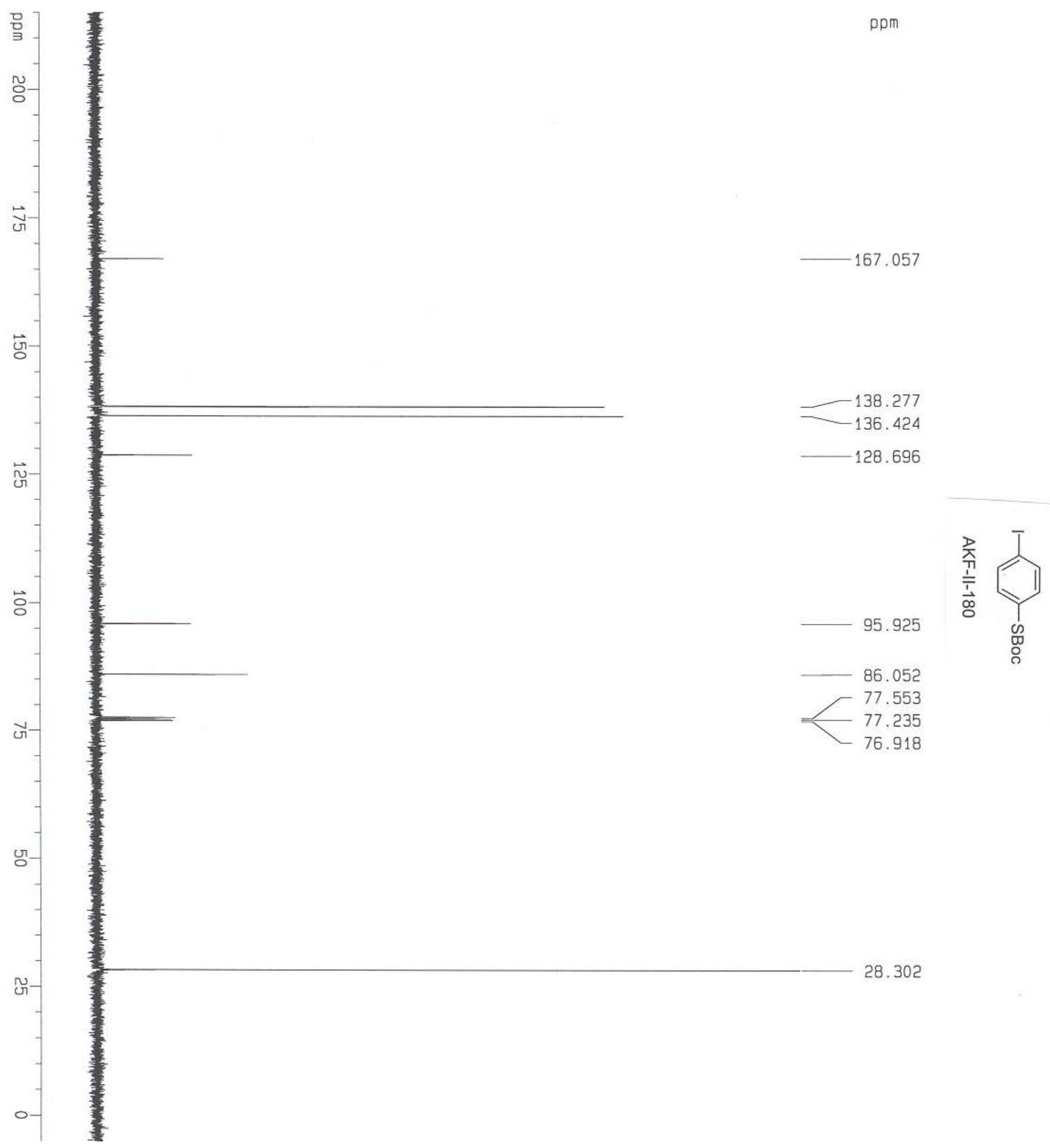

Figure S14: ${ }^{13} \mathrm{C}$ NMR of 7 . 
Flatt, Yao, Maya, and Tour, Orthogonally Functionalized Oligomers, Supporting Information

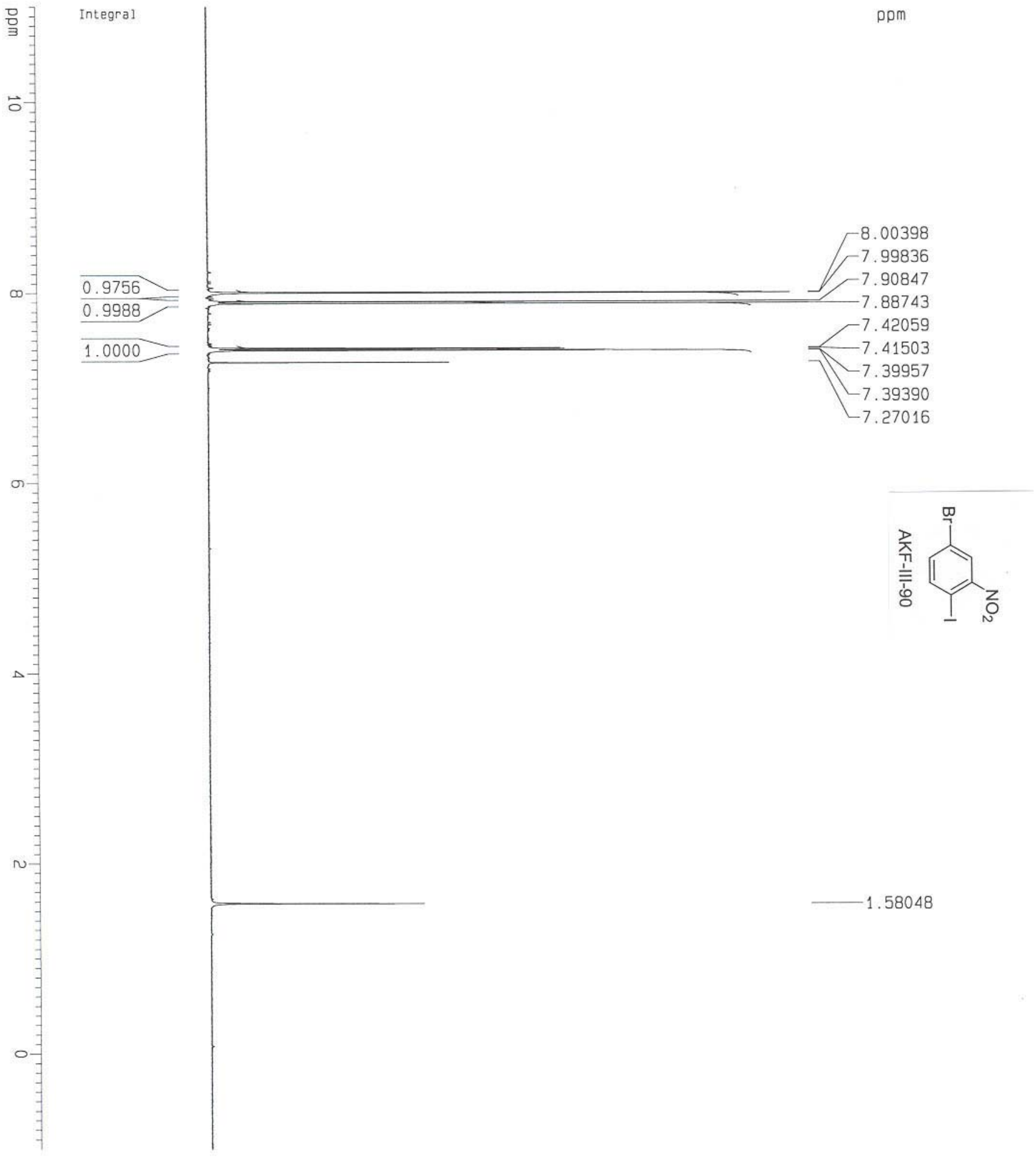

Figure S15: ${ }^{1} \mathrm{H}$ NMR of 8 . 
Flatt, Yao, Maya, and Tour, Orthogonally Functionalized Oligomers, Supporting Information

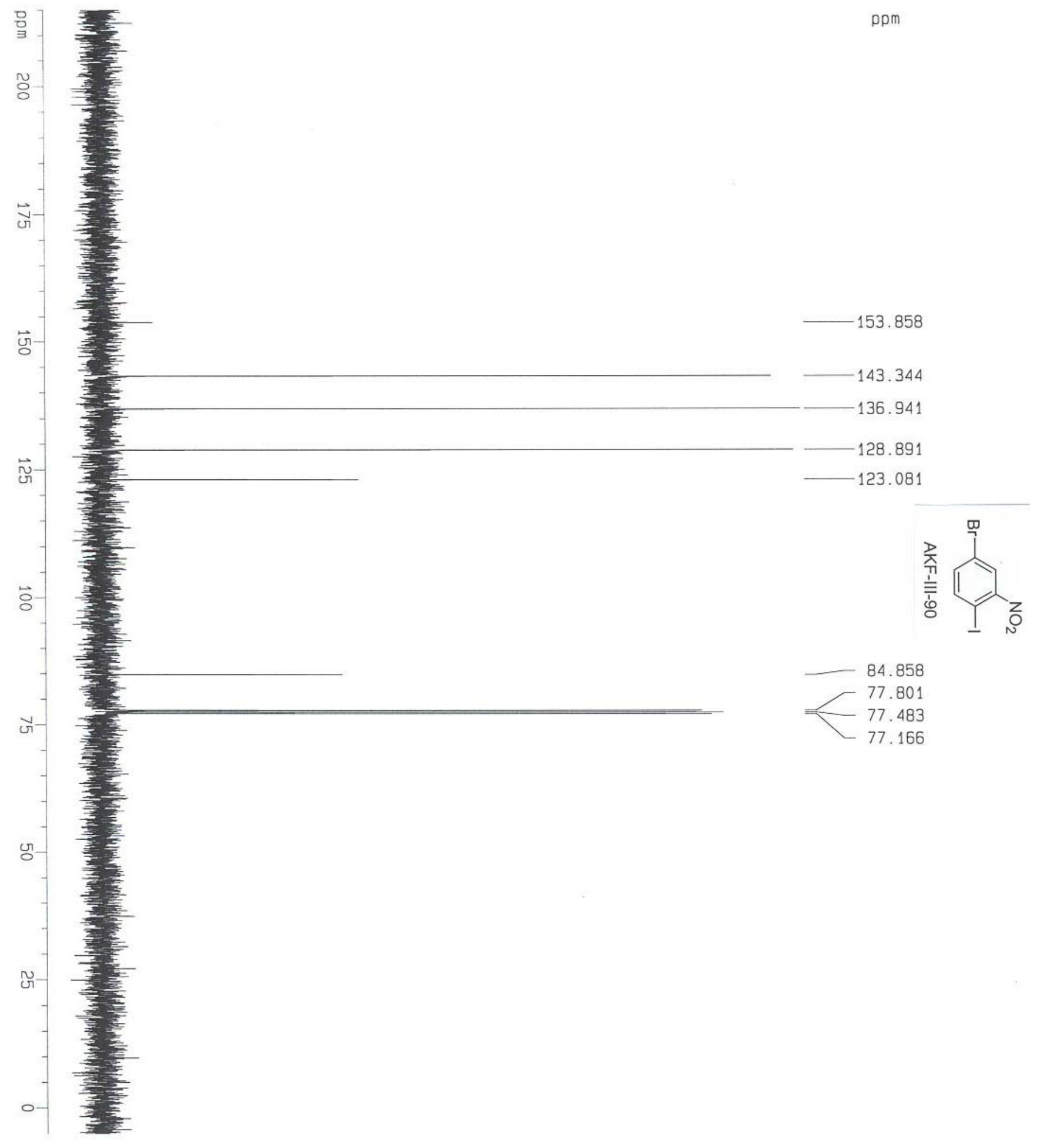

Figure S16: ${ }^{13} \mathrm{C}$ NMR of 8 . 
Flatt, Yao, Maya, and Tour, Orthogonally Functionalized Oligomers, Supporting Information

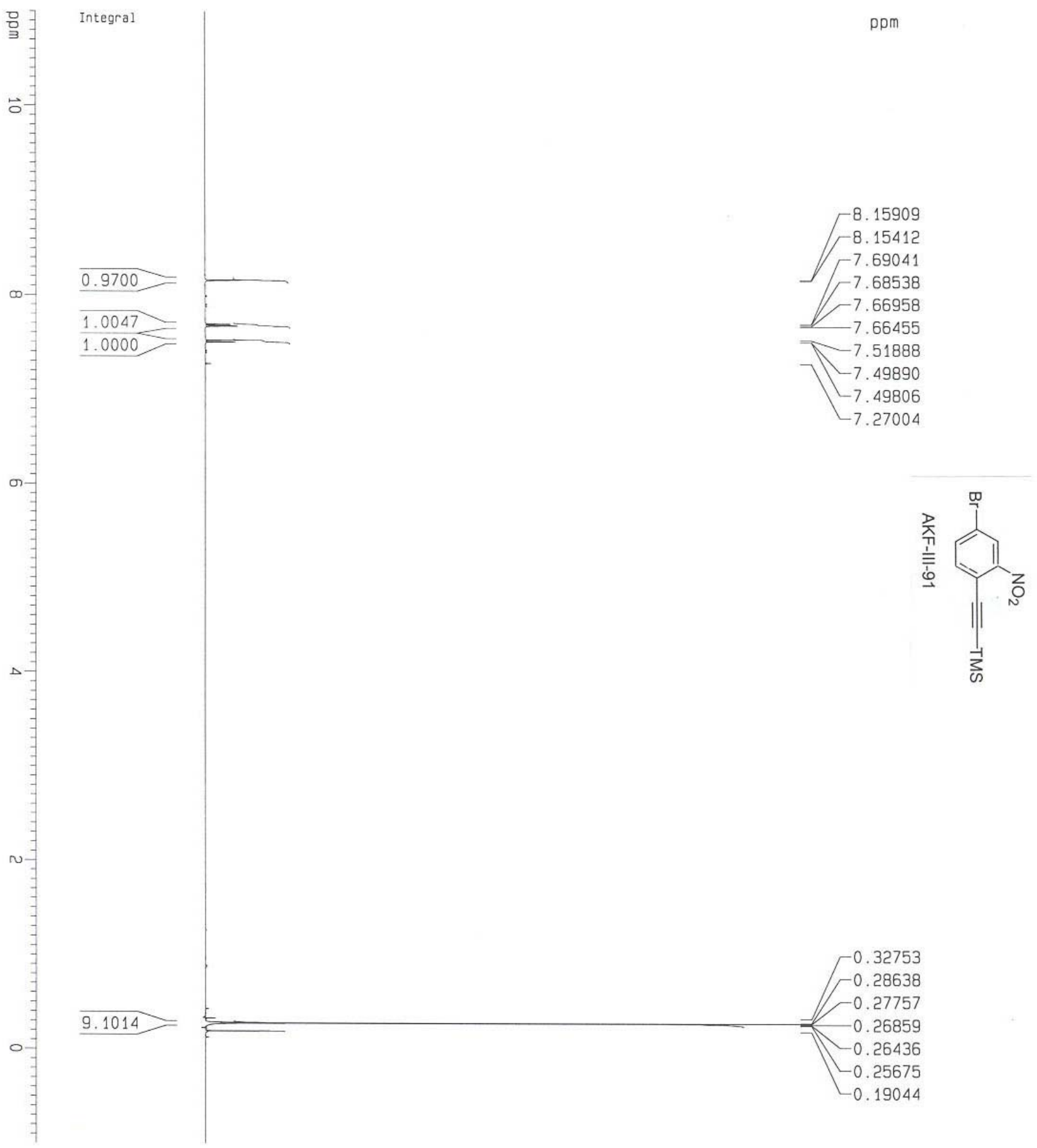

Figure S17: ${ }^{1} \mathrm{H}$ NMR of 9. 
Flatt, Yao, Maya, and Tour, Orthogonally Functionalized Oligomers, Supporting Information

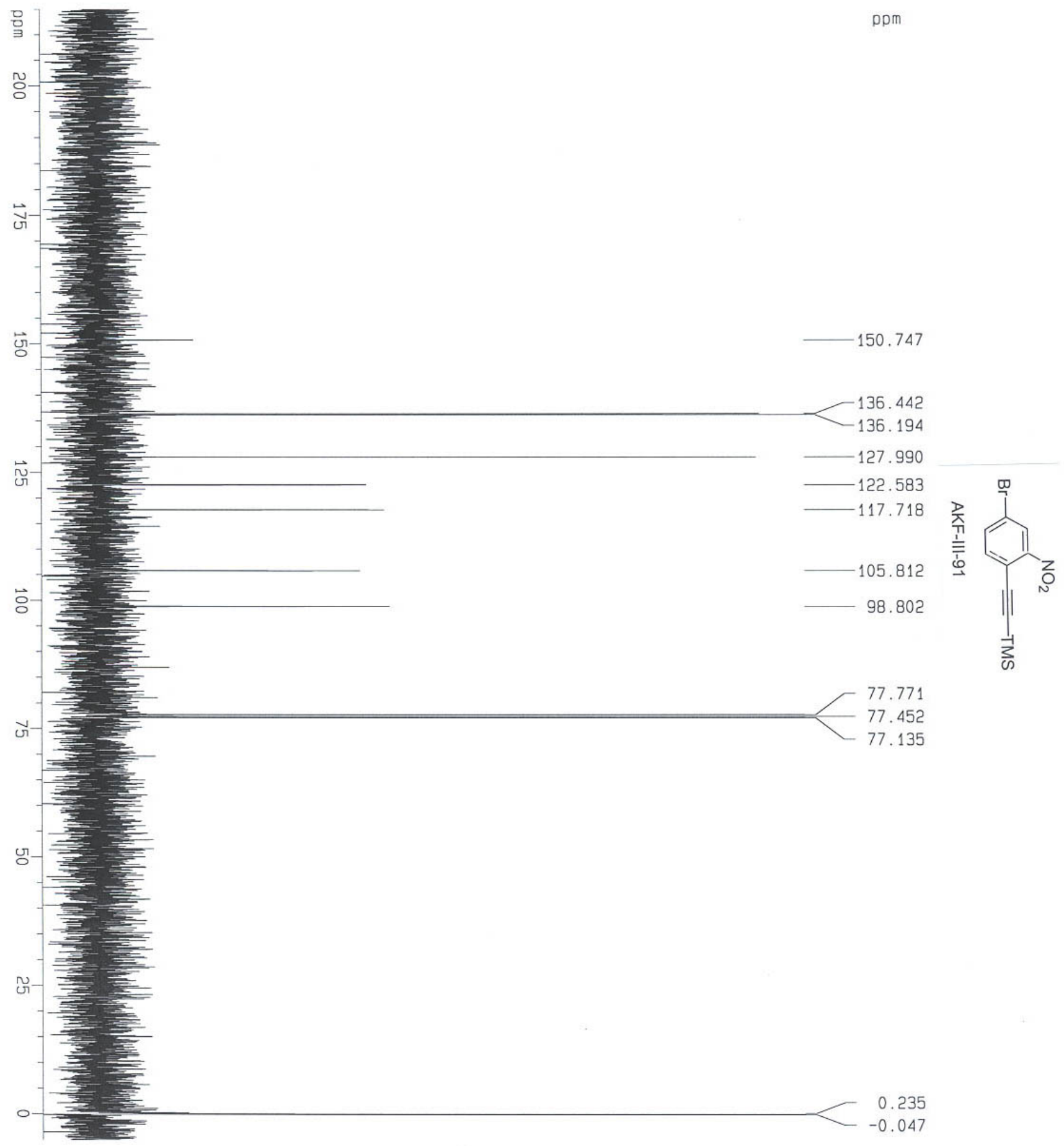

Figure S18: ${ }^{13} \mathrm{C}$ NMR of 9 . 
Flatt, Yao, Maya, and Tour, Orthogonally Functionalized Oligomers, Supporting Information

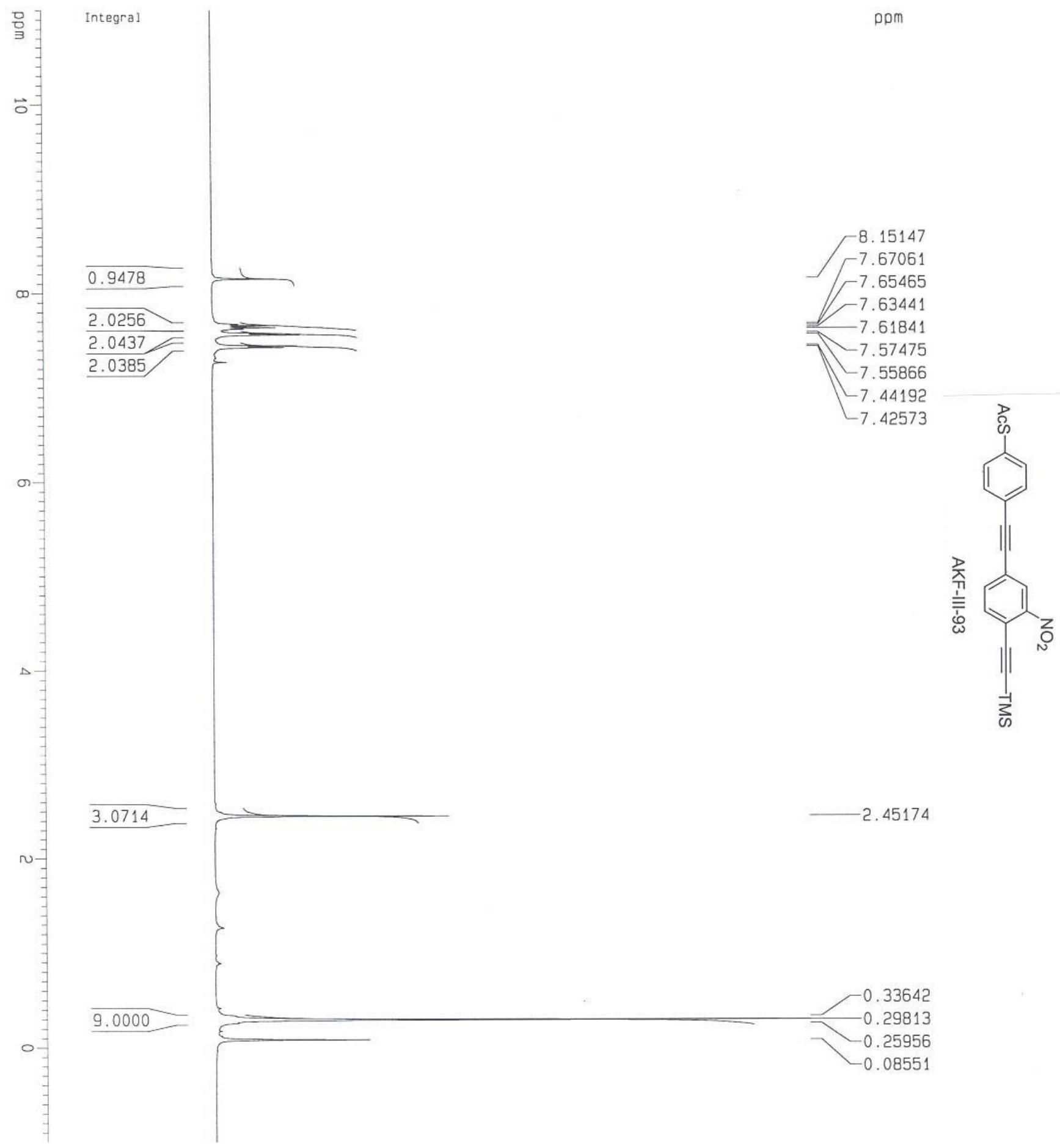

Figure S19: ${ }^{1} \mathrm{H}$ NMR of $\mathbf{1 0 .}$ 
Flatt, Yao, Maya, and Tour, Orthogonally Functionalized Oligomers, Supporting Information

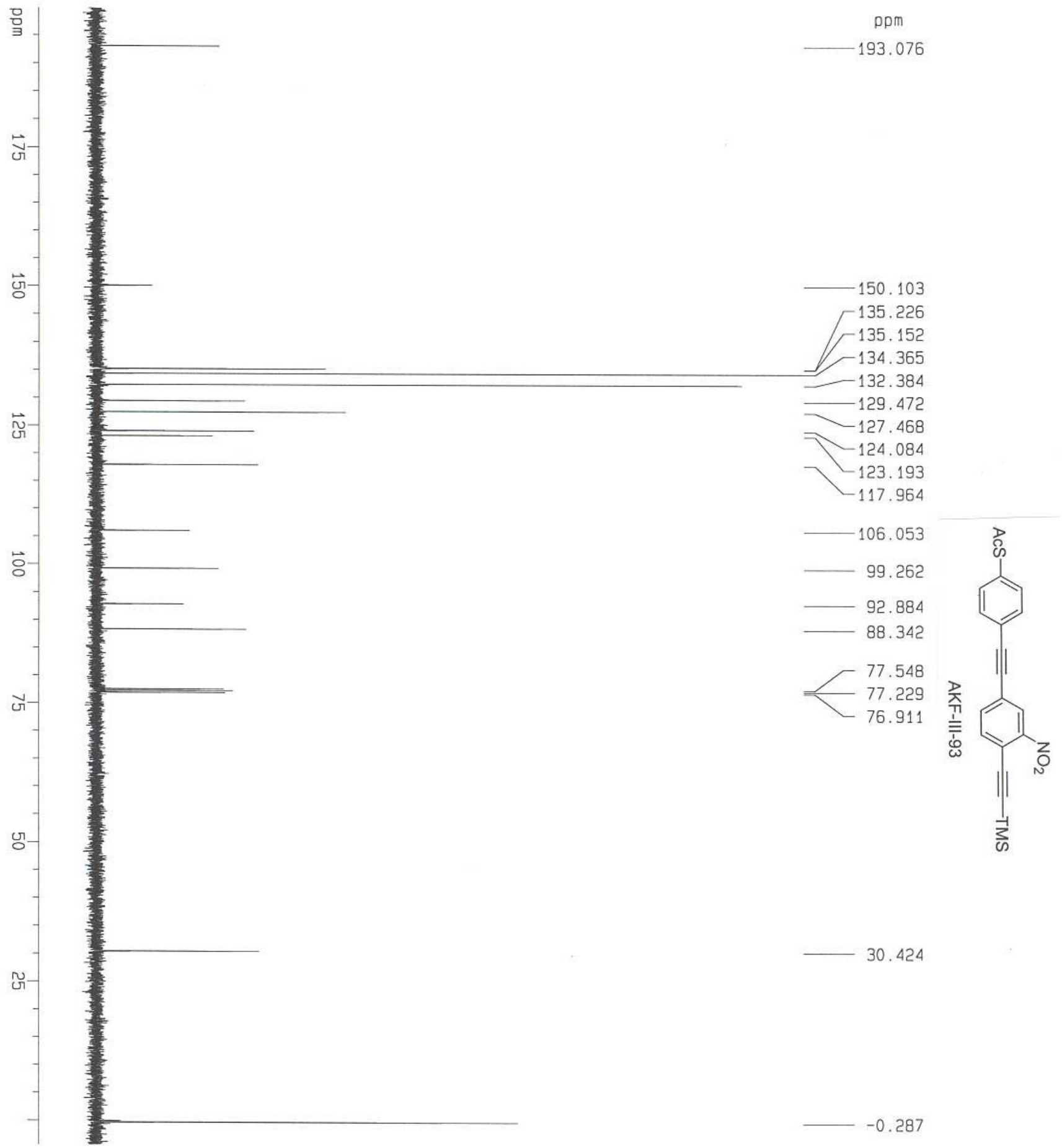

Figure S20: ${ }^{13} \mathrm{C}$ NMR of $\mathbf{1 0}$. 
Flatt, Yao, Maya, and Tour, Orthogonally Functionalized Oligomers, Supporting Information

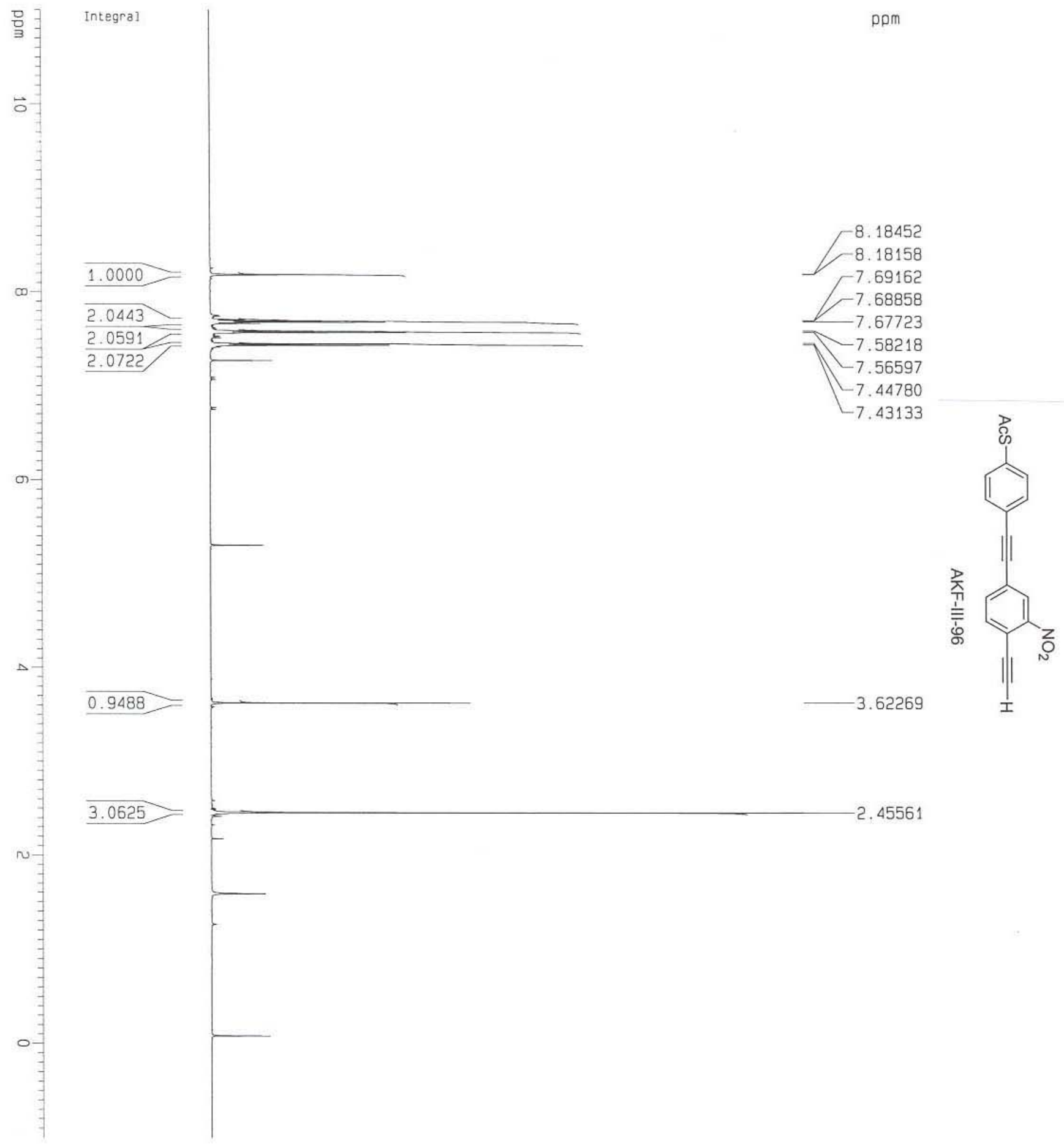

Figure S21: ${ }^{1} \mathrm{H}$ NMR of 11. 
Flatt, Yao, Maya, and Tour, Orthogonally Functionalized Oligomers, Supporting Information

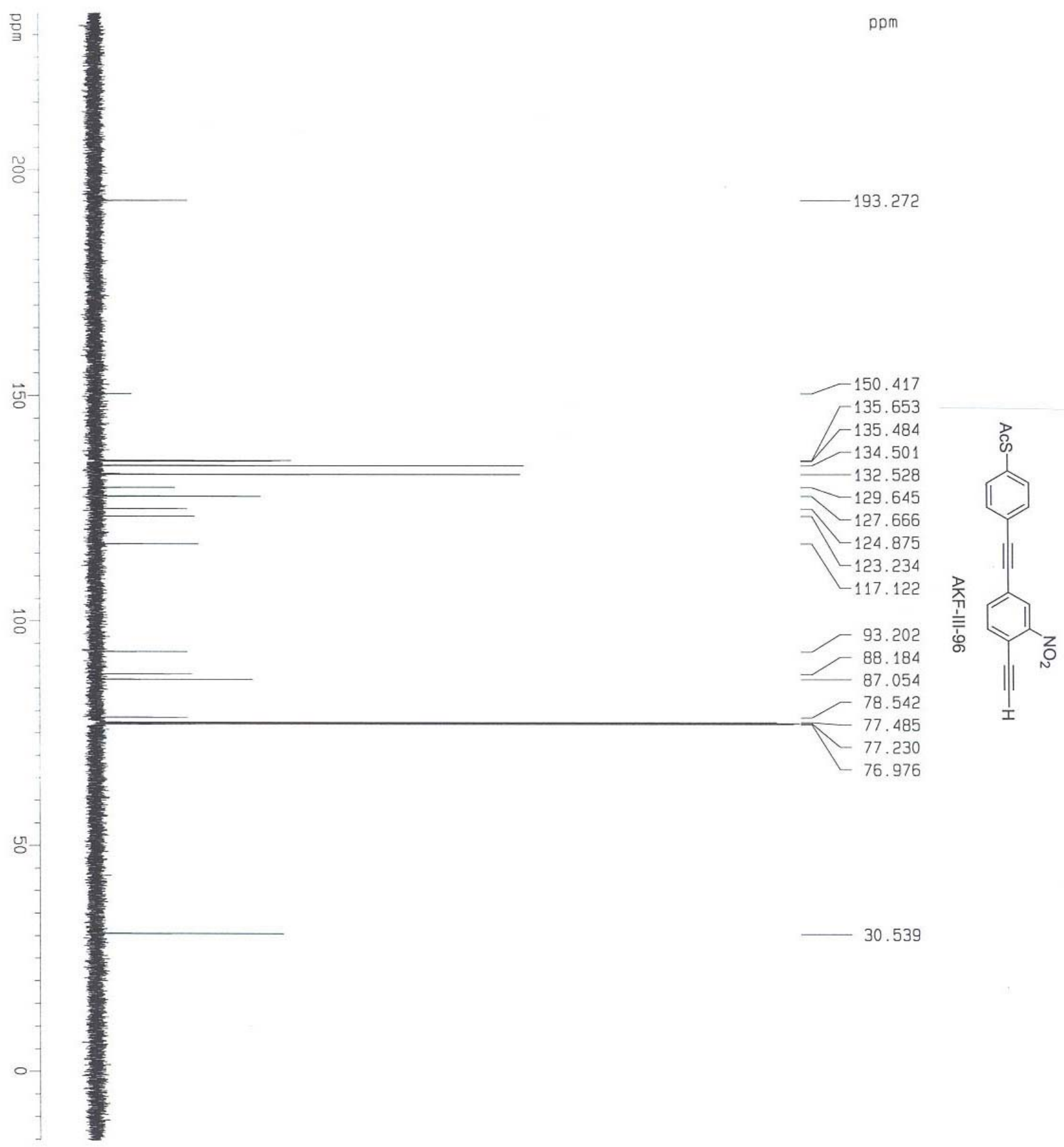

Figure S22: ${ }^{13} \mathrm{C}$ NMR of 11. 
Flatt, Yao, Maya, and Tour, Orthogonally Functionalized Oligomers, Supporting Information

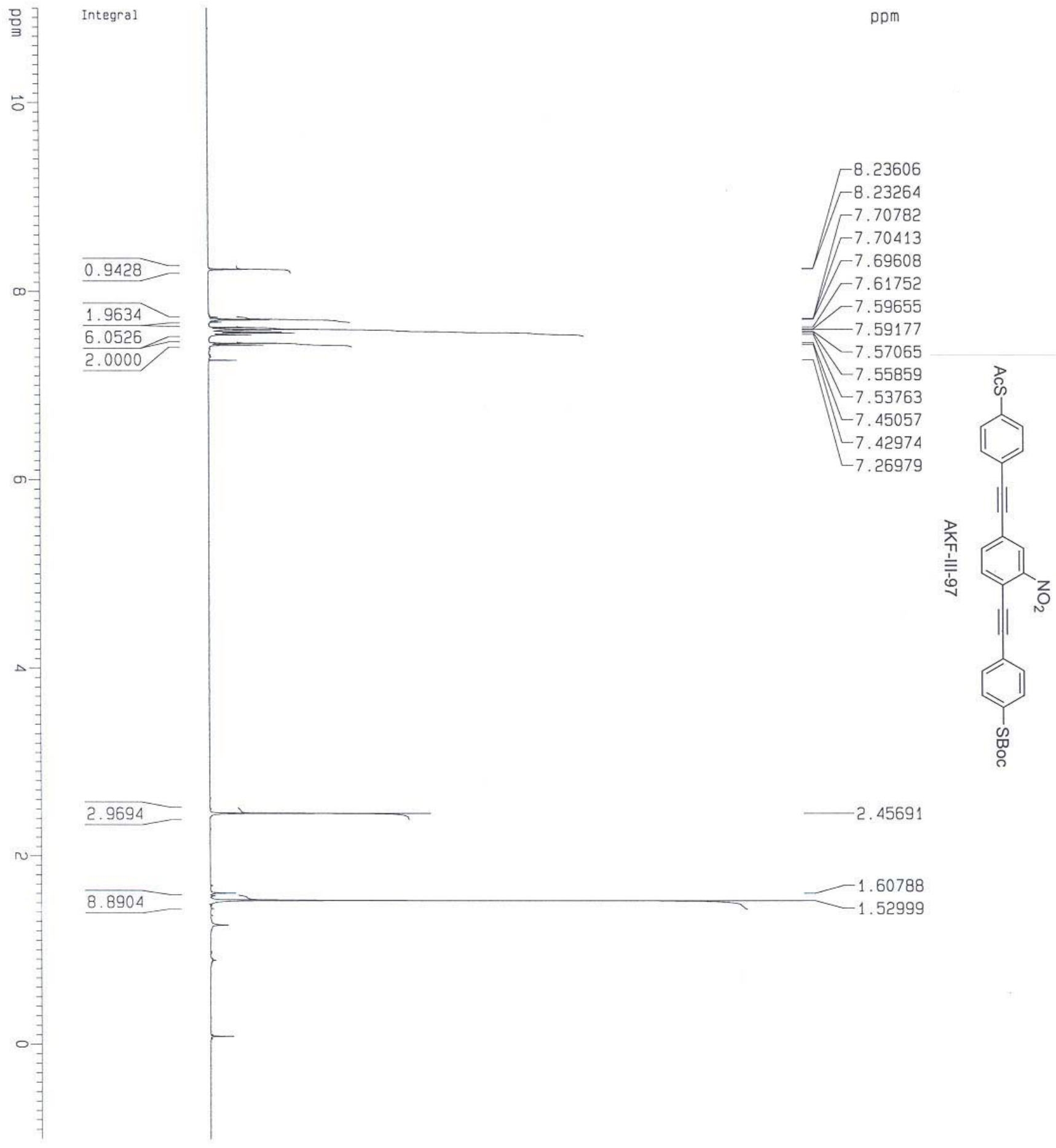

Figure S23: ${ }^{1} \mathrm{H}$ NMR of 12. 
Flatt, Yao, Maya, and Tour, Orthogonally Functionalized Oligomers, Supporting Information

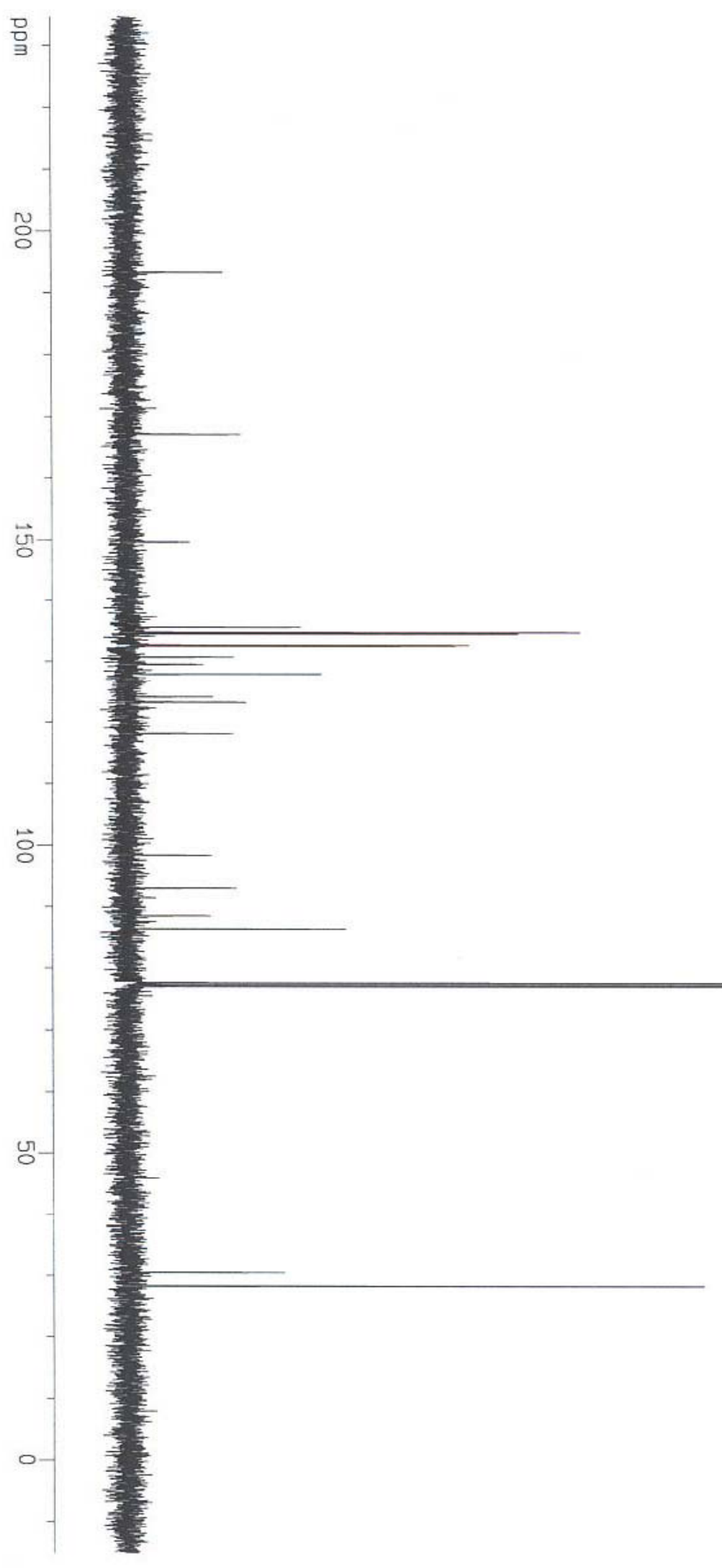

ppm

193.320

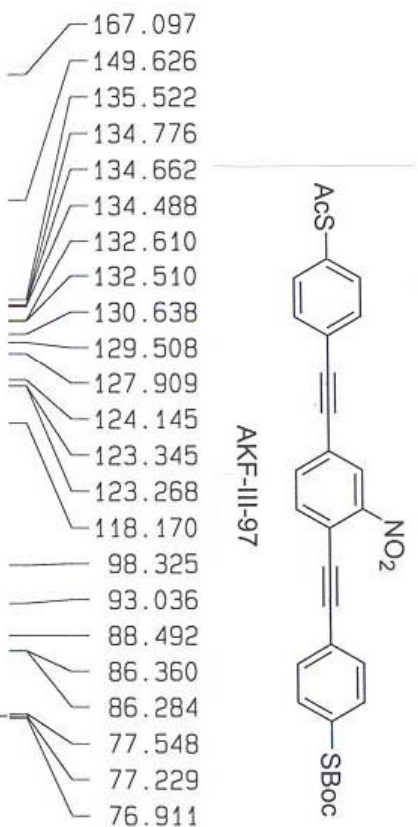

30.537

28.362

Figure S24: ${ }^{13} \mathrm{C}$ NMR of 12. 
Flatt, Yao, Maya, and Tour, Orthogonally Functionalized Oligomers, Supporting Information

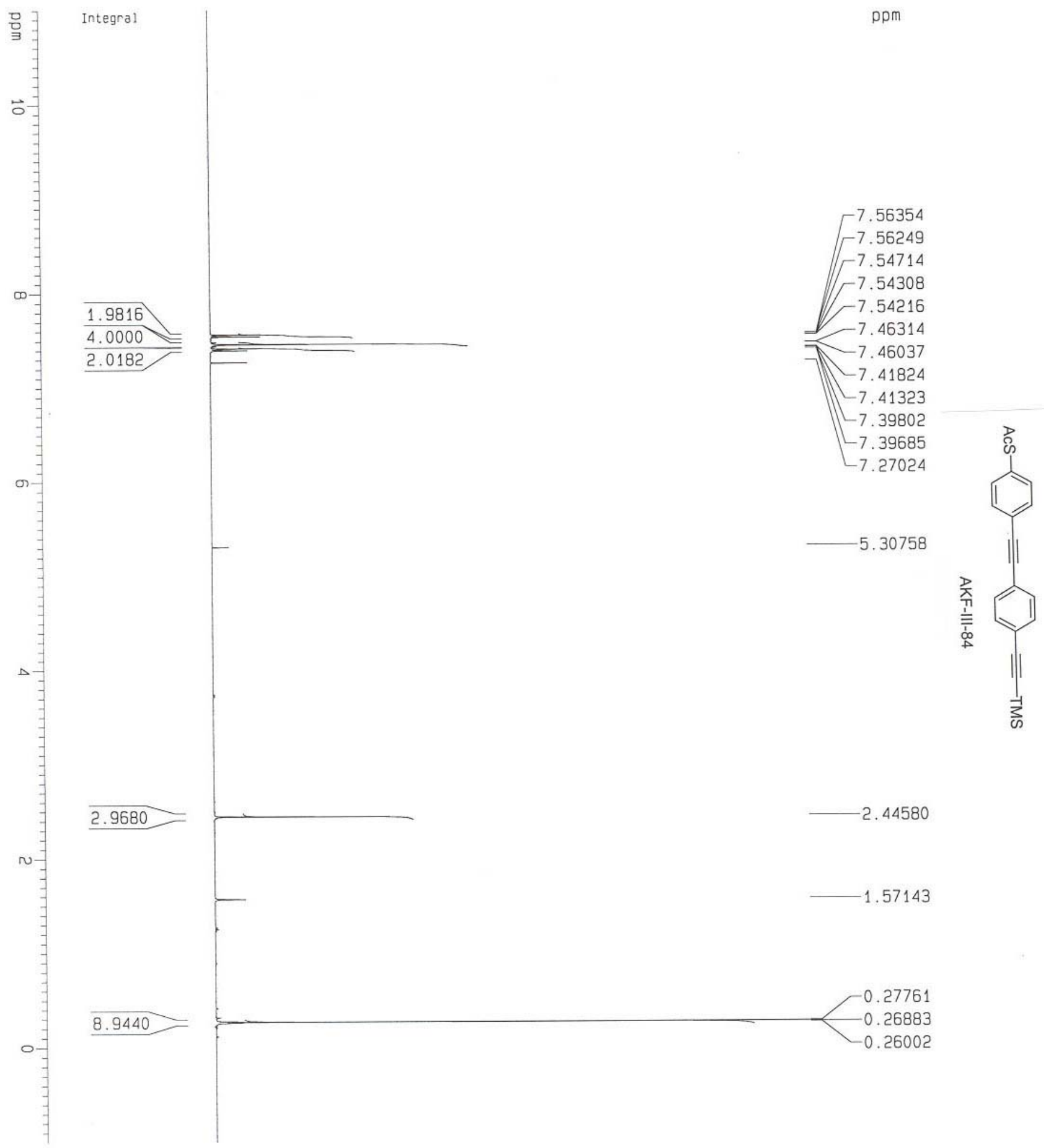

Figure S25: ${ }^{1} \mathrm{H}$ NMR of 14. 
Flatt, Yao, Maya, and Tour, Orthogonally Functionalized Oligomers, Supporting Information

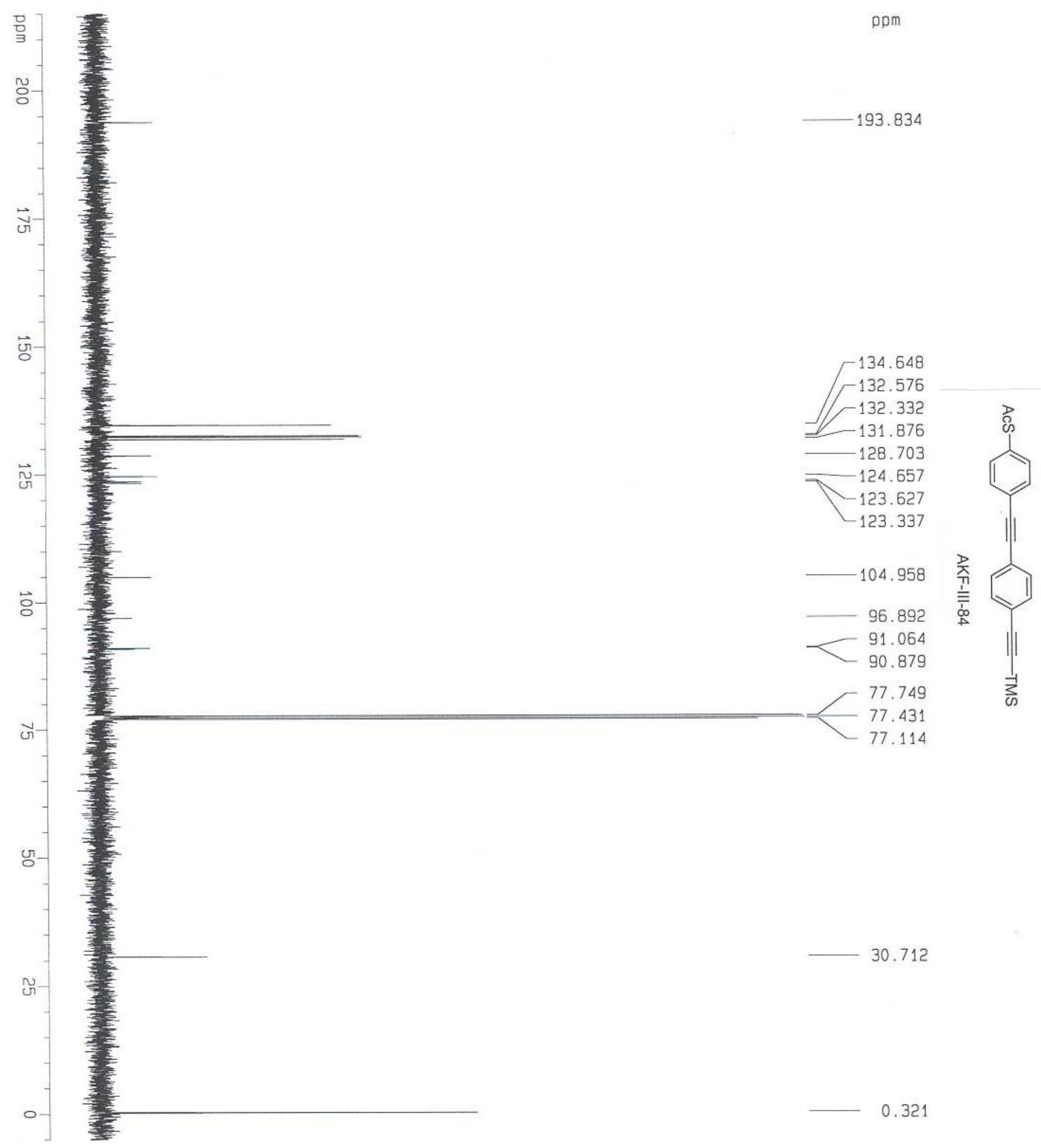

Figure S26: ${ }^{13} \mathrm{C}$ NMR of 14. 
Flatt, Yao, Maya, and Tour, Orthogonally Functionalized Oligomers, Supporting Information

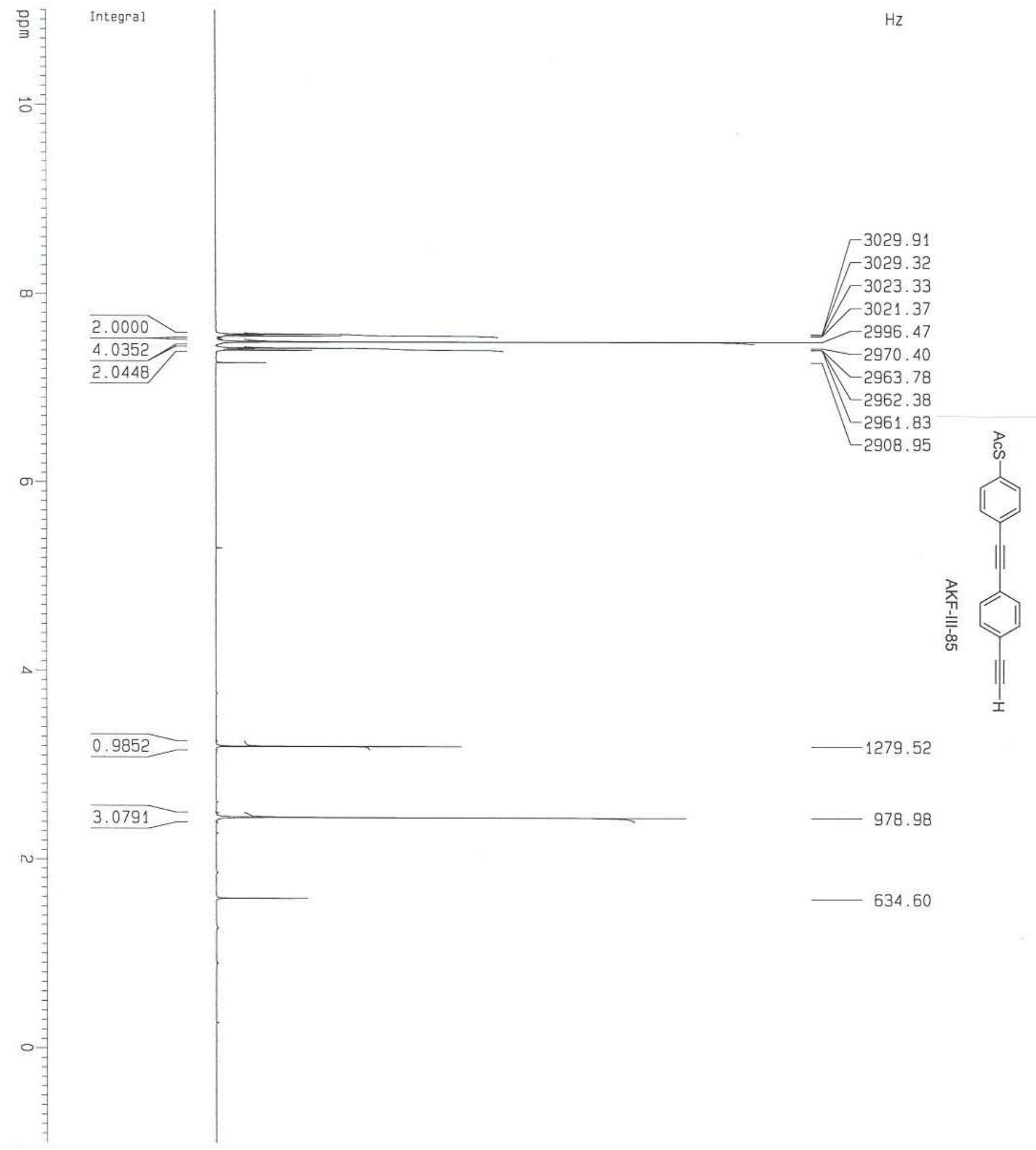

Figure S27: ${ }^{1} \mathrm{H}$ NMR of 15. 
Flatt, Yao, Maya, and Tour, Orthogonally Functionalized Oligomers, Supporting Information

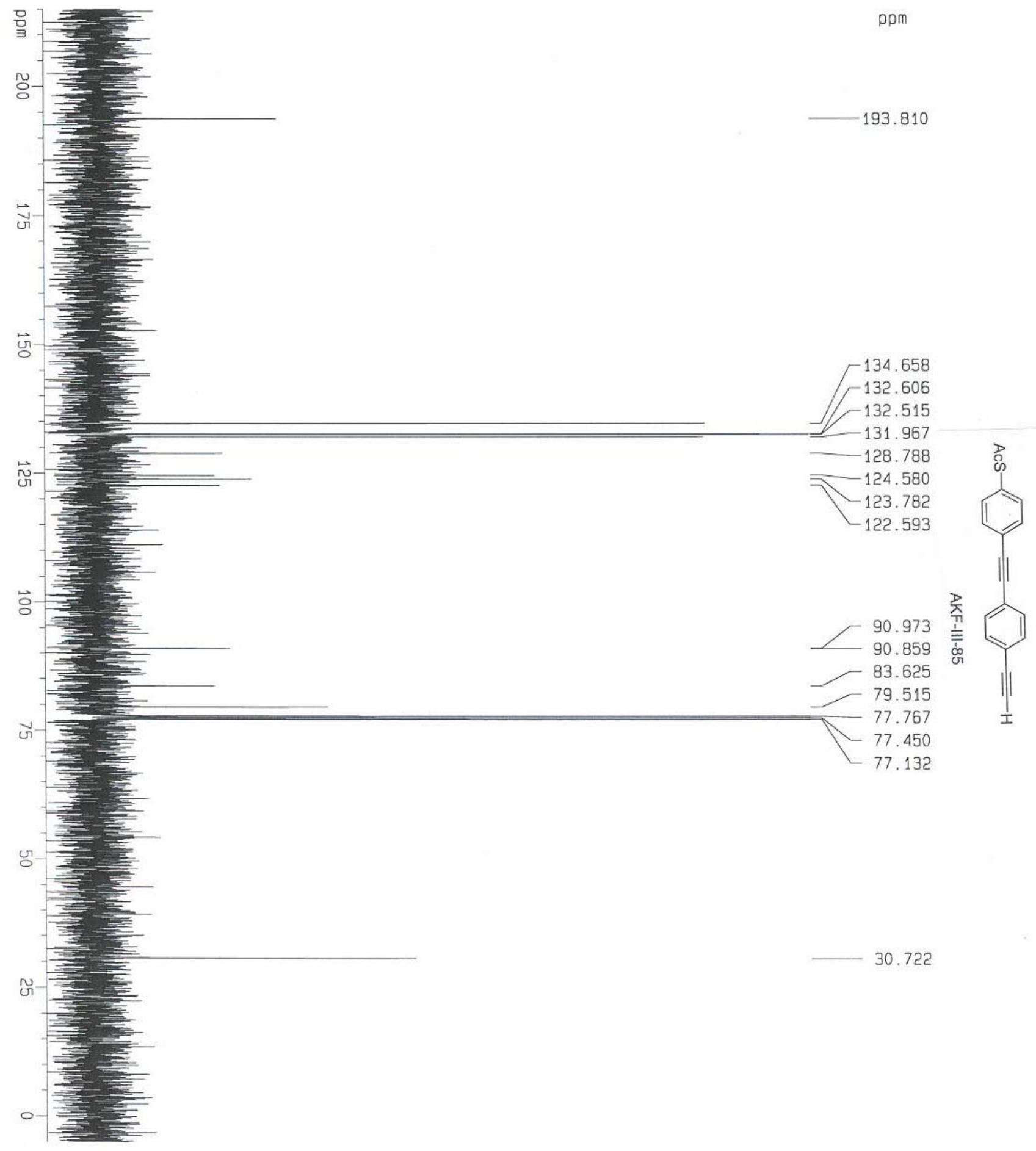

Figure S28: ${ }^{13} \mathrm{C}$ NMR of $\mathbf{1 5}$. 
Flatt, Yao, Maya, and Tour, Orthogonally Functionalized Oligomers, Supporting Information

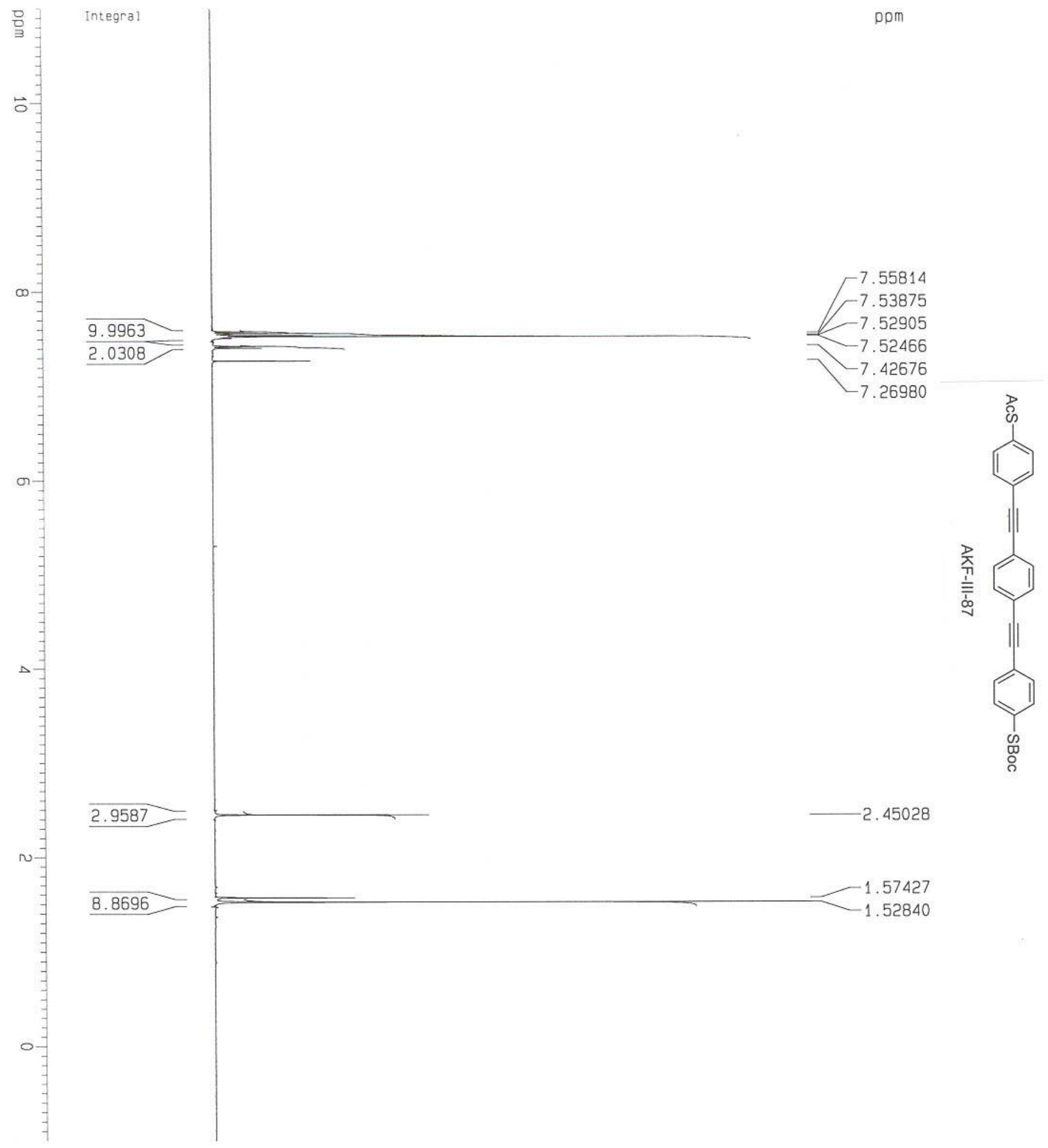

Figure S29: ${ }^{1} \mathrm{H}$ NMR of 16. 
Flatt, Yao, Maya, and Tour, Orthogonally Functionalized Oligomers, Supporting Information

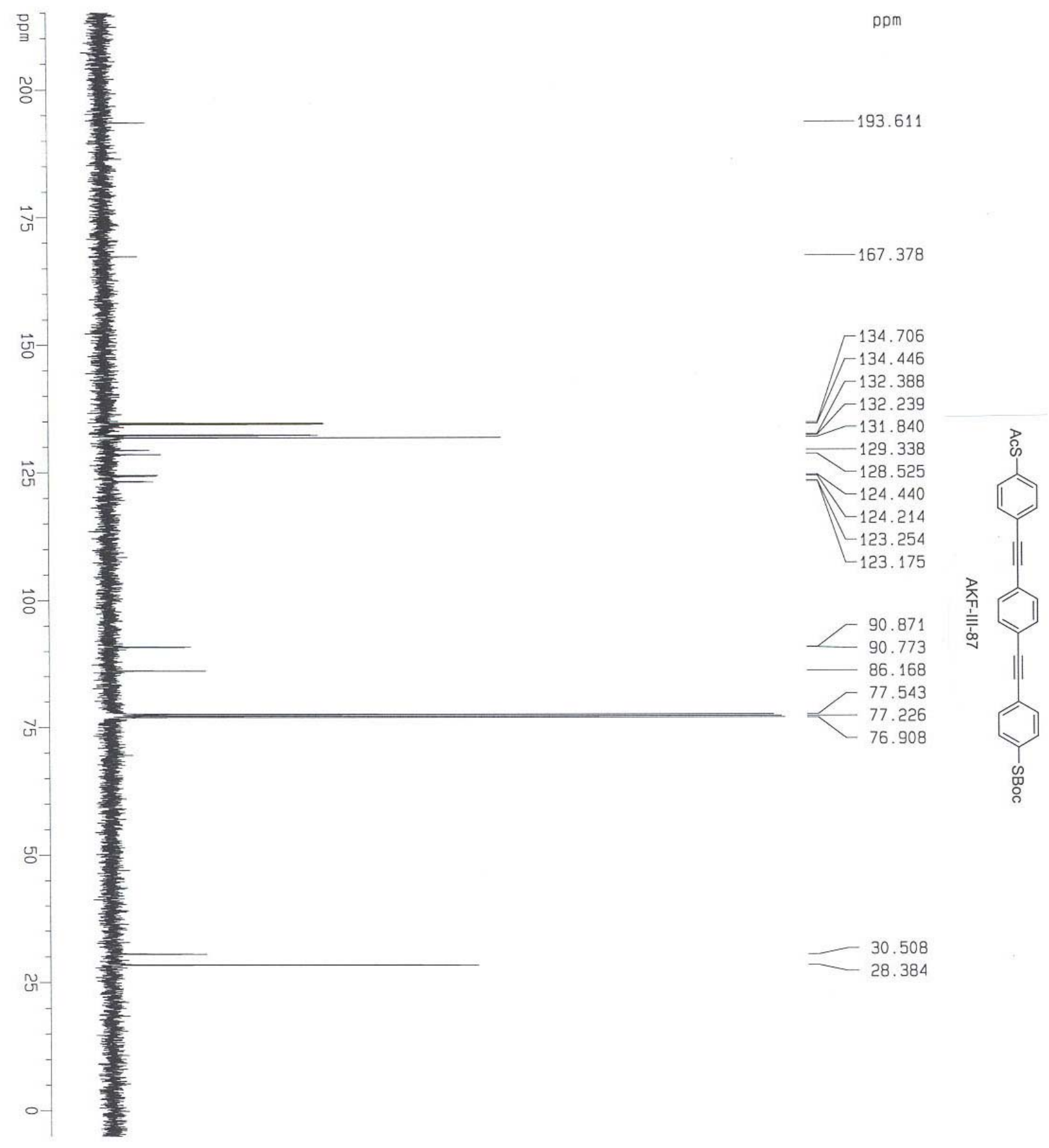

Figure S30: ${ }^{13} \mathrm{C}$ NMR of 16 . 
Flatt, Yao, Maya, and Tour, Orthogonally Functionalized Oligomers, Supporting Information

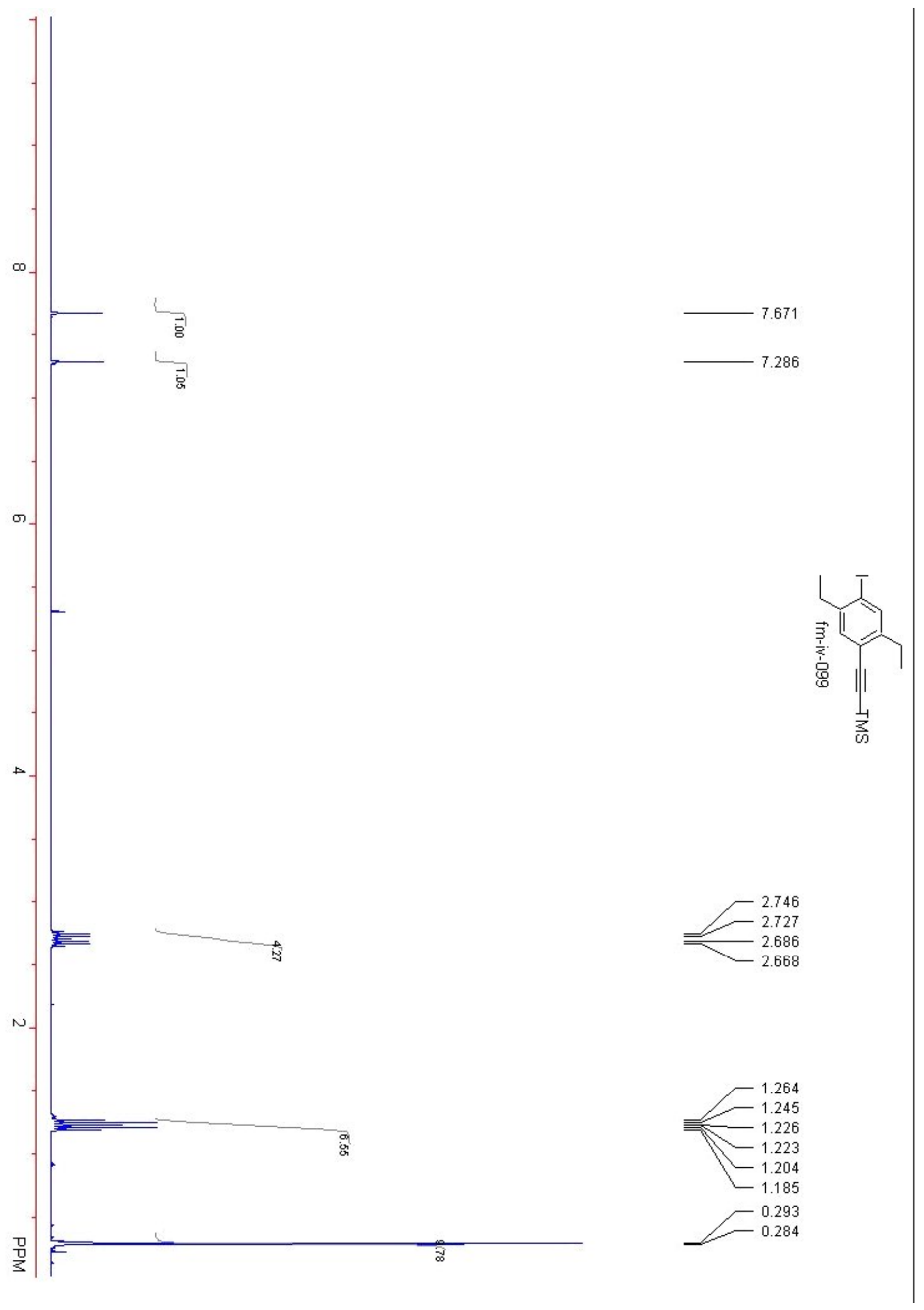

Figure S31: ${ }^{1} \mathrm{H}$ NMR of 17. 
Flatt, Yao, Maya, and Tour, Orthogonally Functionalized Oligomers, Supporting Information
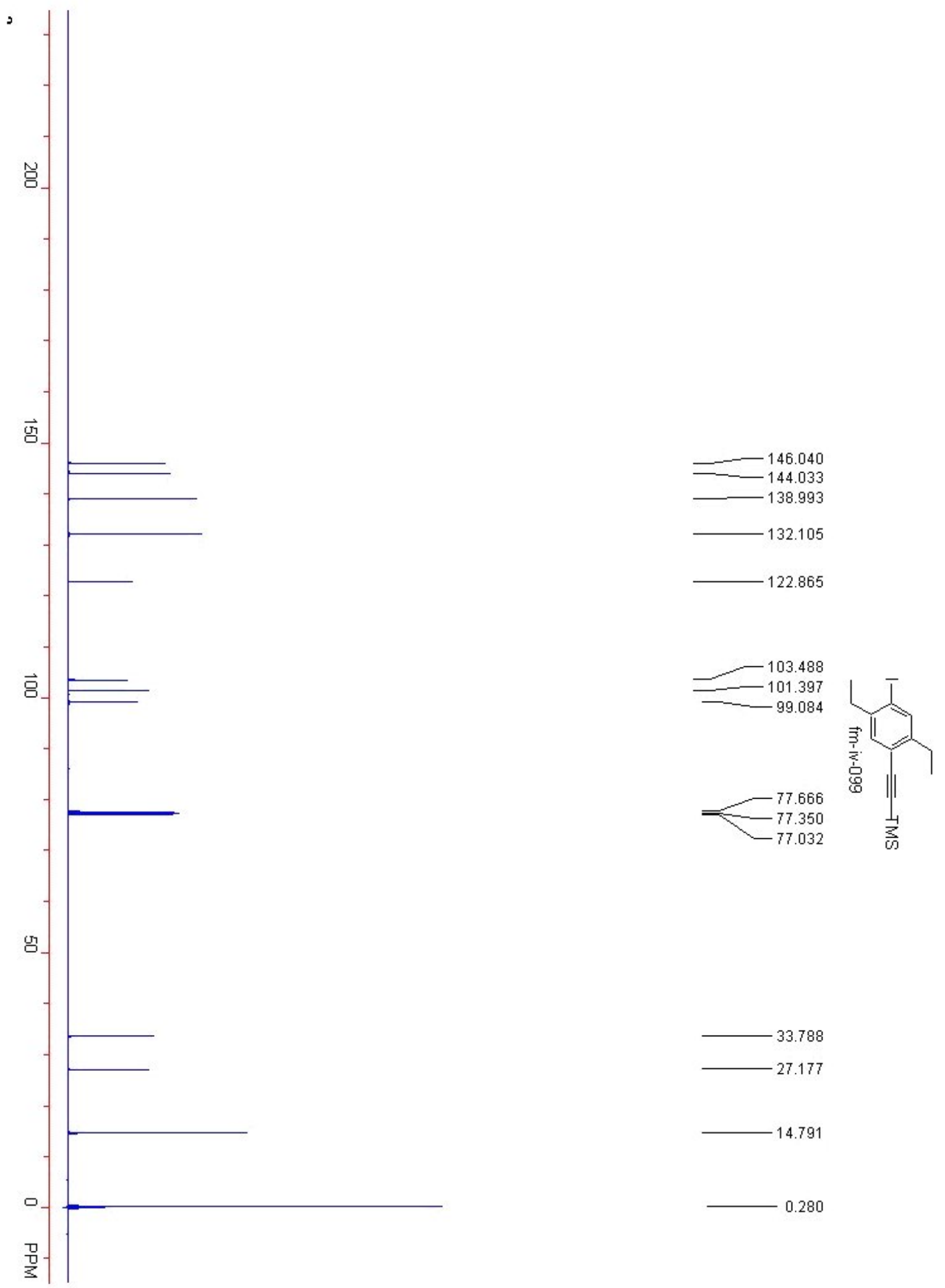

Figure S32: ${ }^{13} \mathrm{C}$ NMR of 17. 
Flatt, Yao, Maya, and Tour, Orthogonally Functionalized Oligomers, Supporting Information
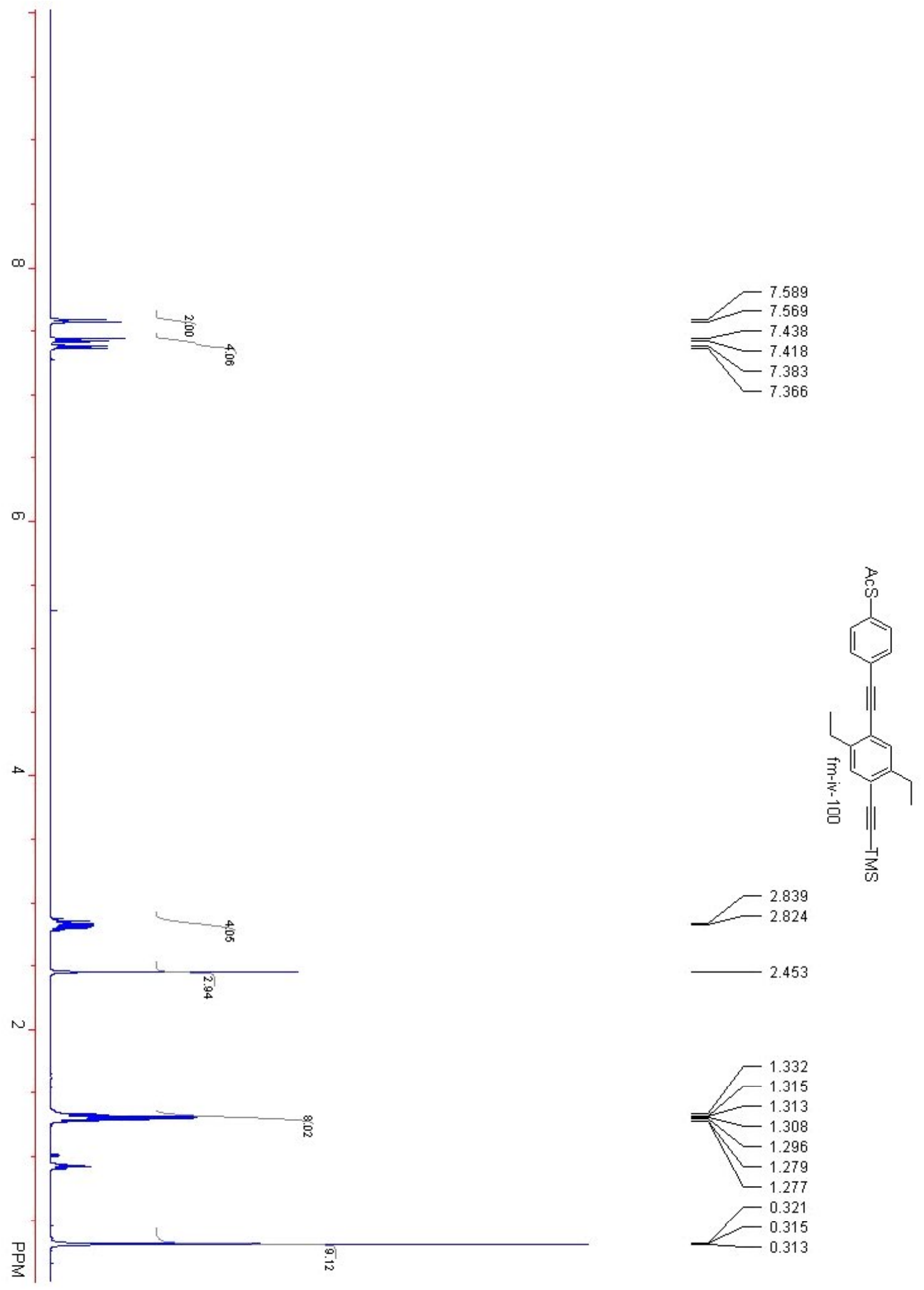

Figure S33: ${ }^{1} \mathrm{H}$ NMR of 18. 
Flatt, Yao, Maya, and Tour, Orthogonally Functionalized Oligomers, Supporting Information

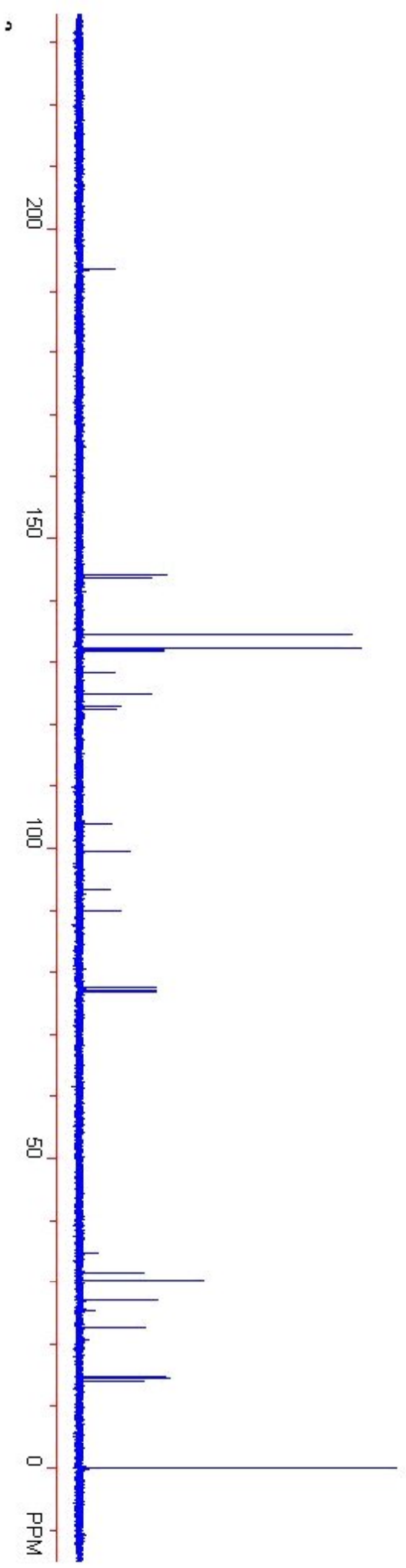

$-193.464$
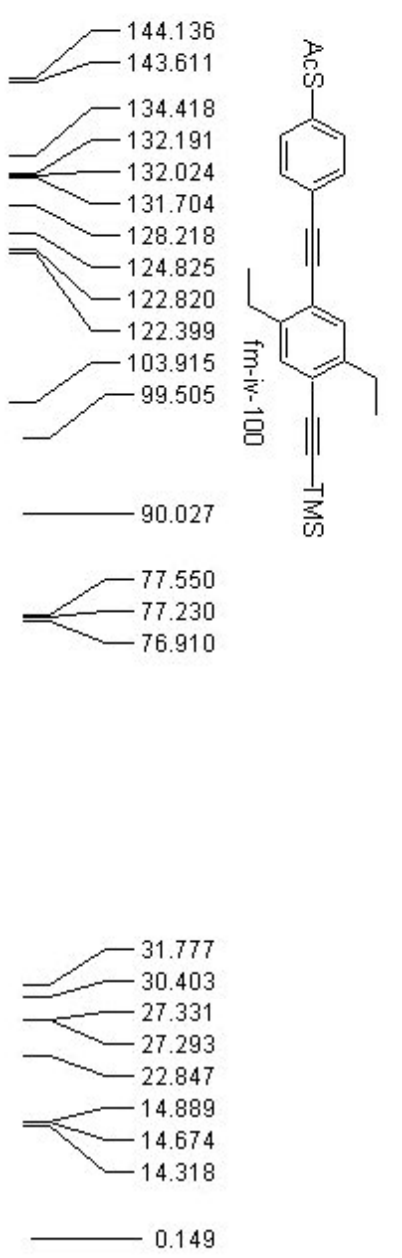

Figure S34: ${ }^{13} \mathrm{C}$ NMR of 18 . 
Flatt, Yao, Maya, and Tour, Orthogonally Functionalized Oligomers, Supporting Information
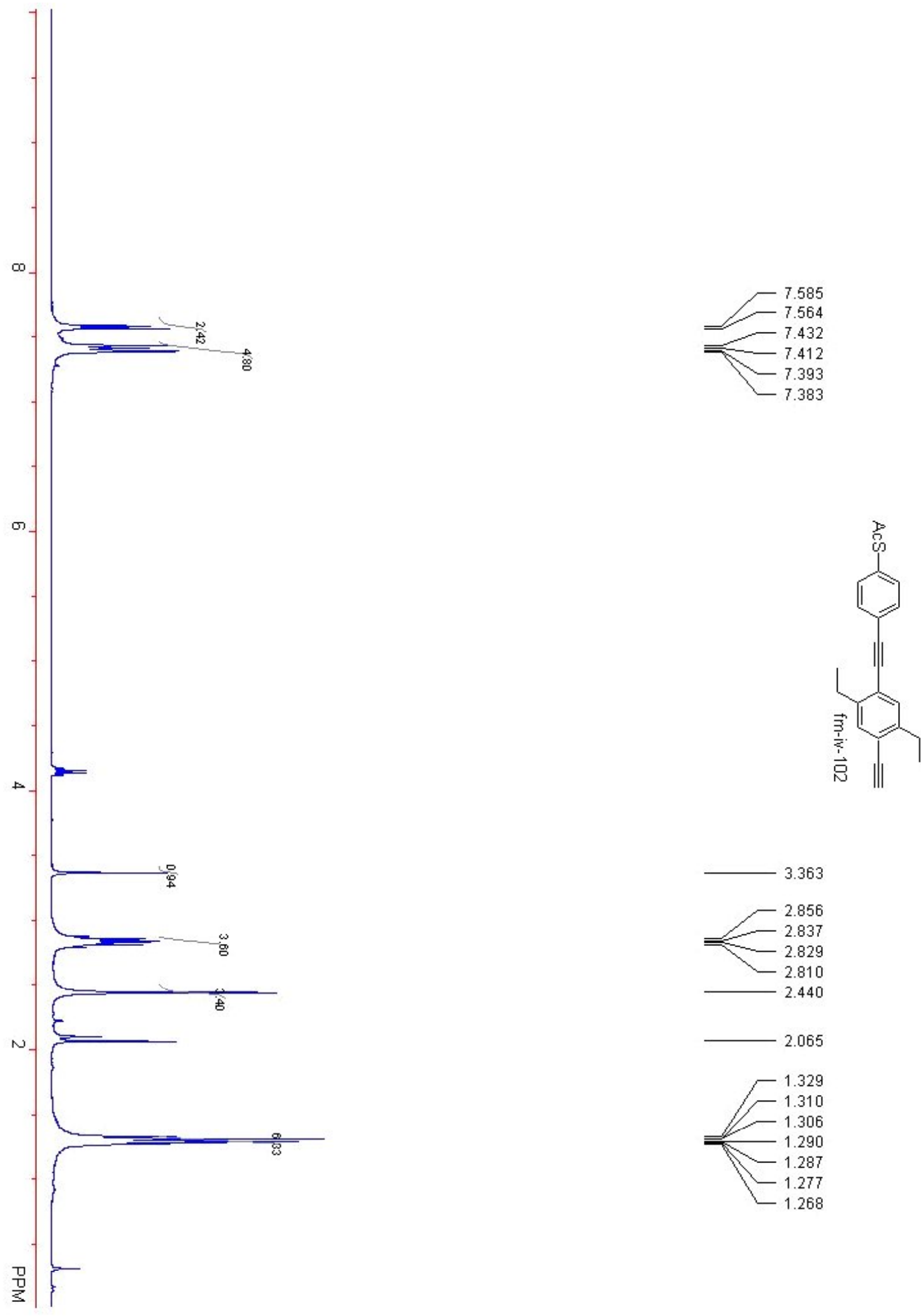

Figure S35: ${ }^{1} \mathrm{H}$ NMR of 19. 
Flatt, Yao, Maya, and Tour, Orthogonally Functionalized Oligomers, Supporting Information
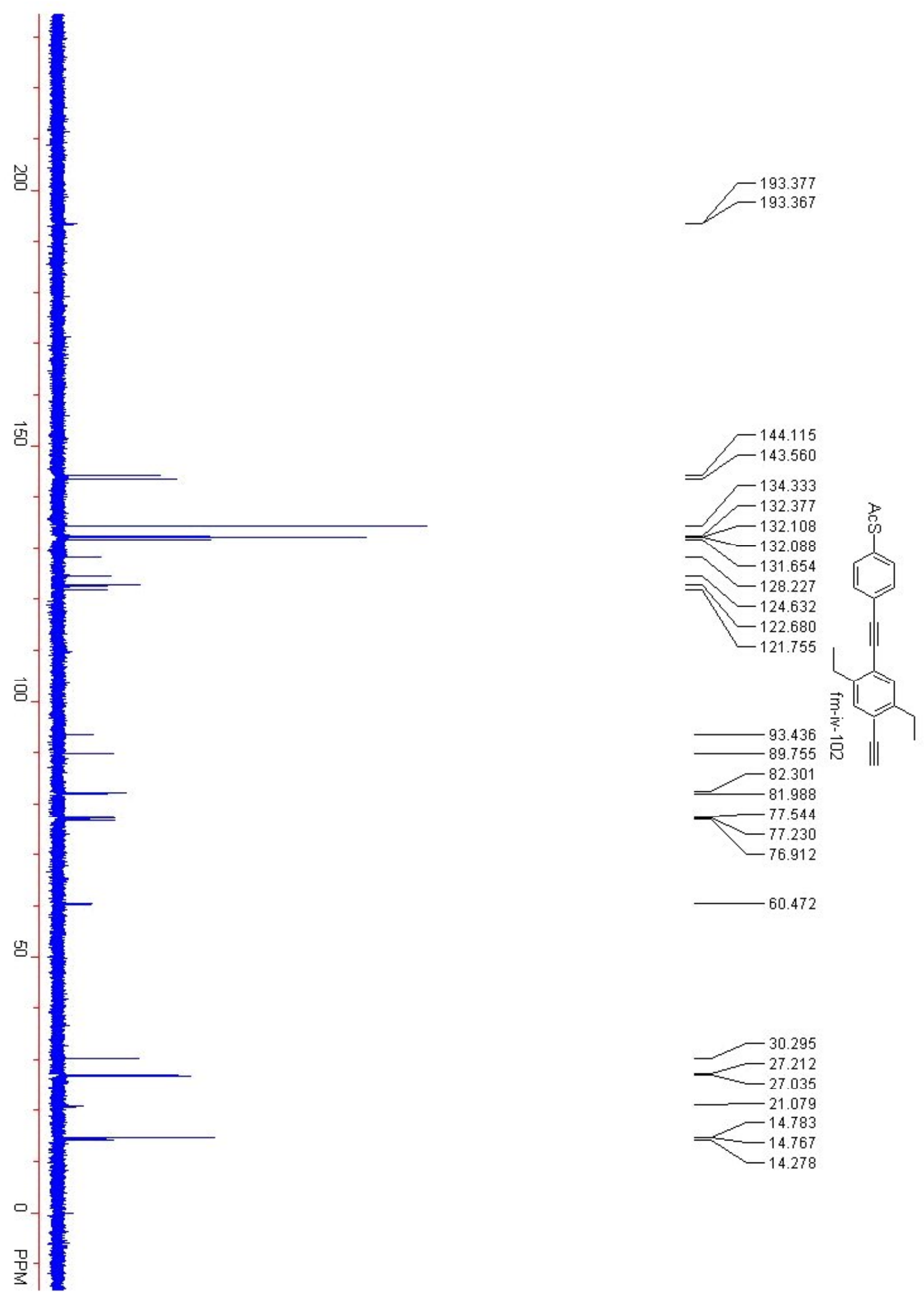

Figure S36: ${ }^{13} \mathrm{C}$ NMR of 19.

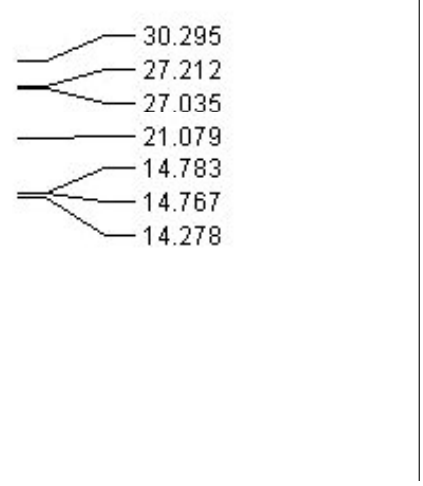


Flatt, Yao, Maya, and Tour, Orthogonally Functionalized Oligomers, Supporting Information

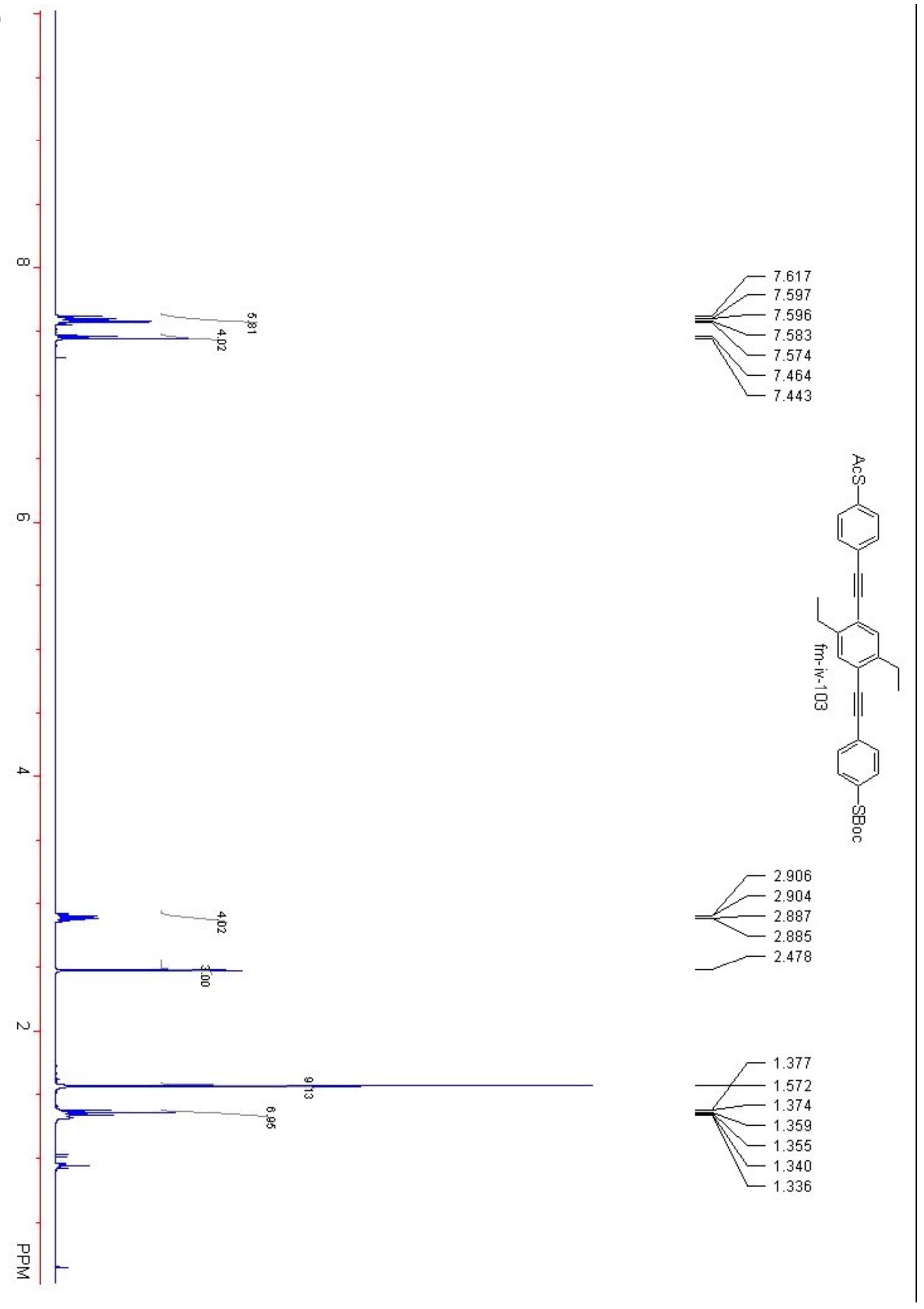

Figure S37: ${ }^{1} \mathrm{H}$ NMR of 20. 
Flatt, Yao, Maya, and Tour, Orthogonally Functionalized Oligomers, Supporting Information
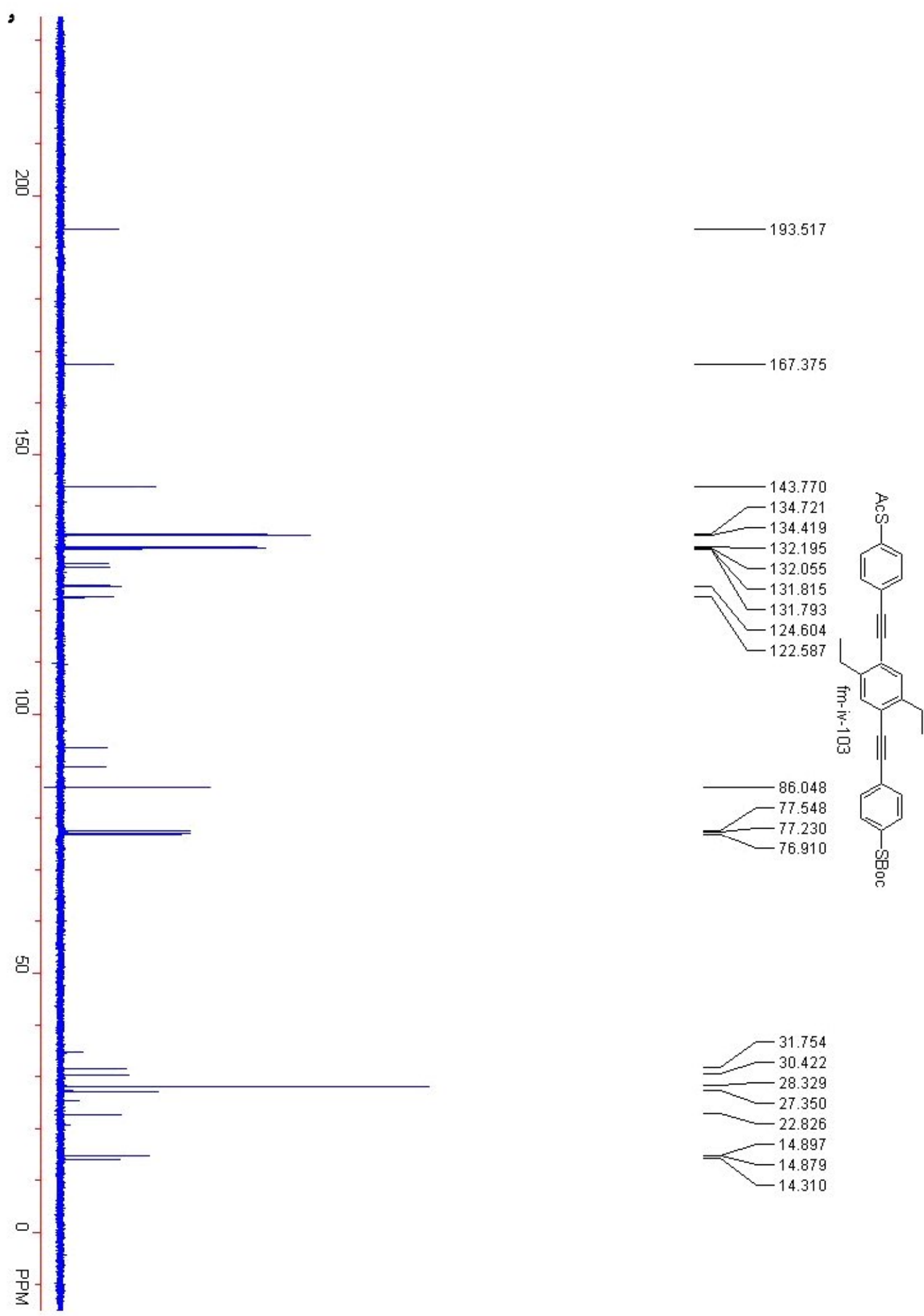

Figure S38: ${ }^{13} \mathrm{C}$ NMR of 20 .

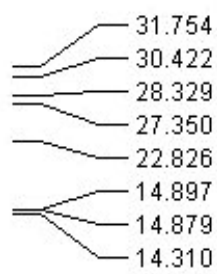


Flatt, Yao, Maya, and Tour, Orthogonally Functionalized Oligomers, Supporting Information

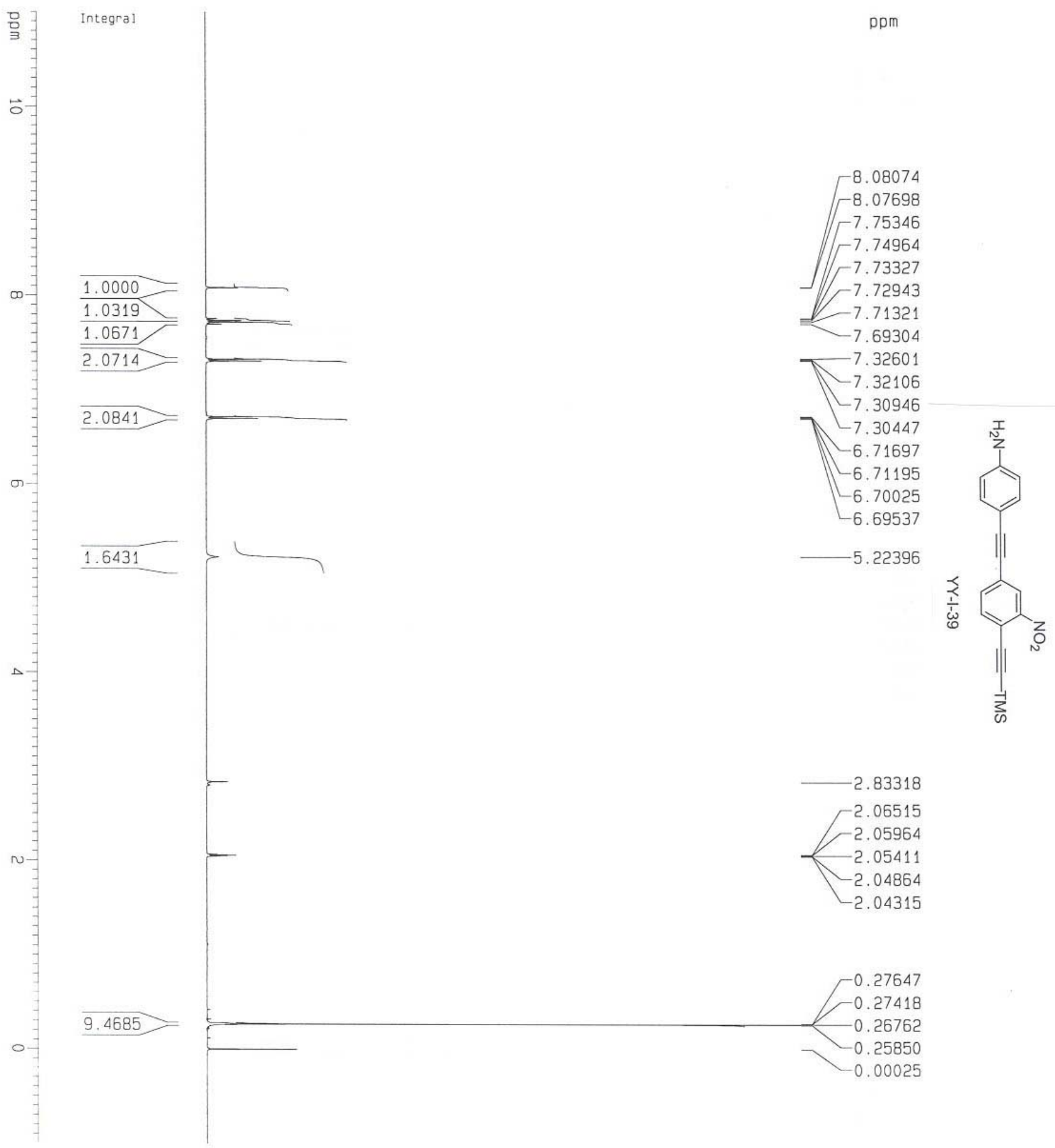

Figure S39: ${ }^{1} \mathrm{H}$ NMR of 24. 
Flatt, Yao, Maya, and Tour, Orthogonally Functionalized Oligomers, Supporting Information

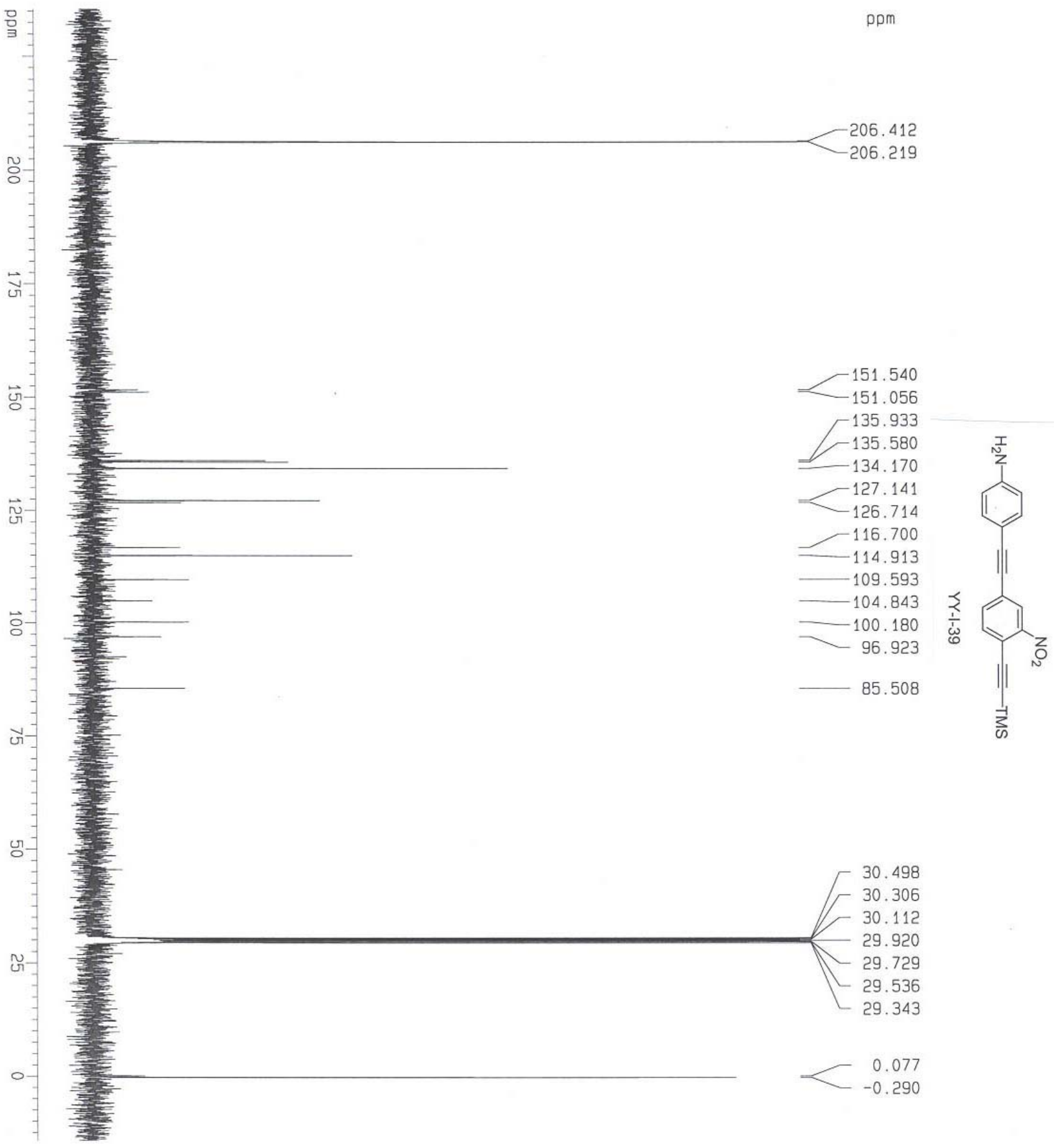

Figure S40: ${ }^{13} \mathrm{C}$ NMR of 24 . 
Flatt, Yao, Maya, and Tour, Orthogonally Functionalized Oligomers, Supporting Information

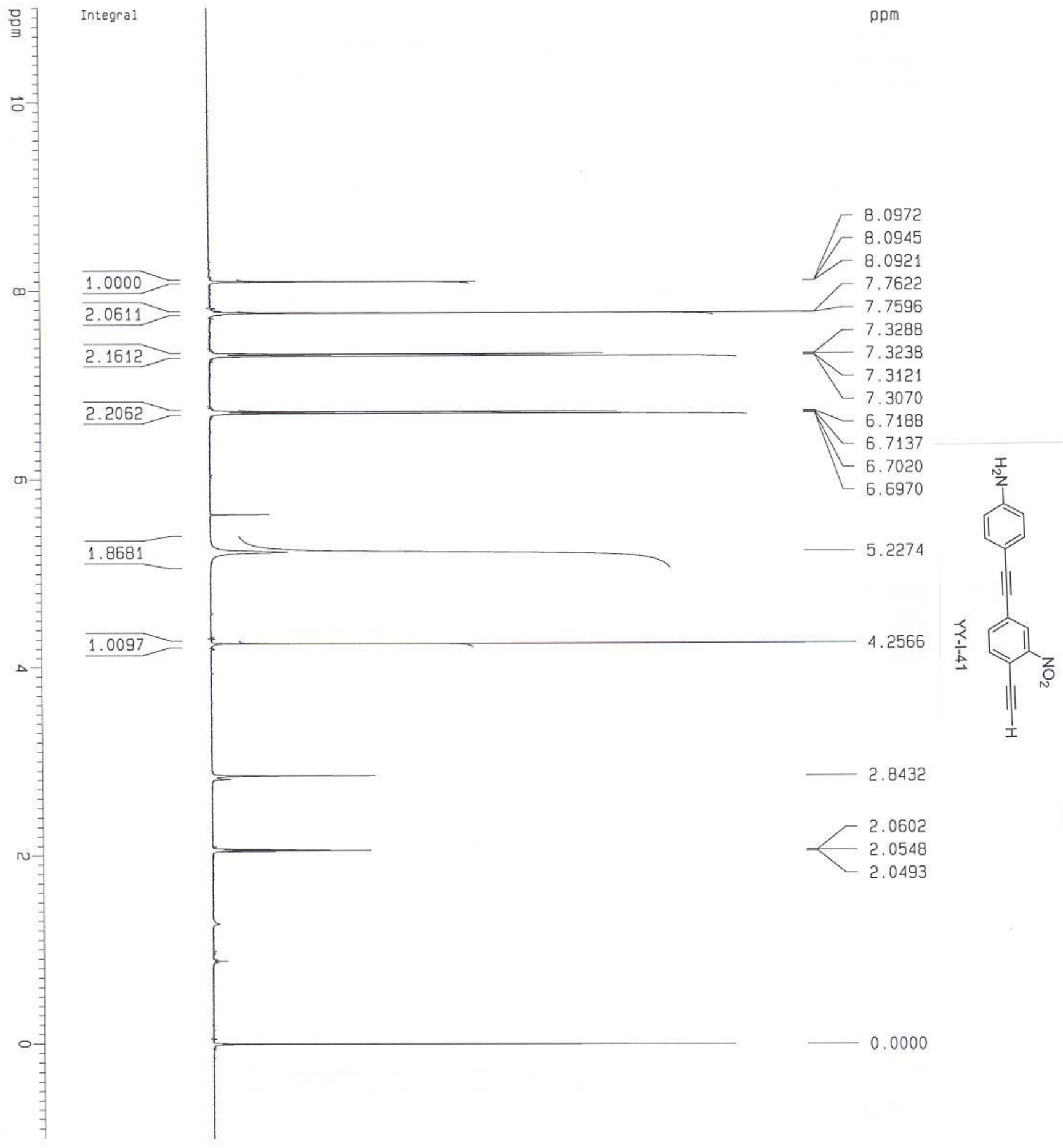

Figure S41: ${ }^{1} \mathrm{H}$ NMR of 25. 
Flatt, Yao, Maya, and Tour, Orthogonally Functionalized Oligomers, Supporting Information

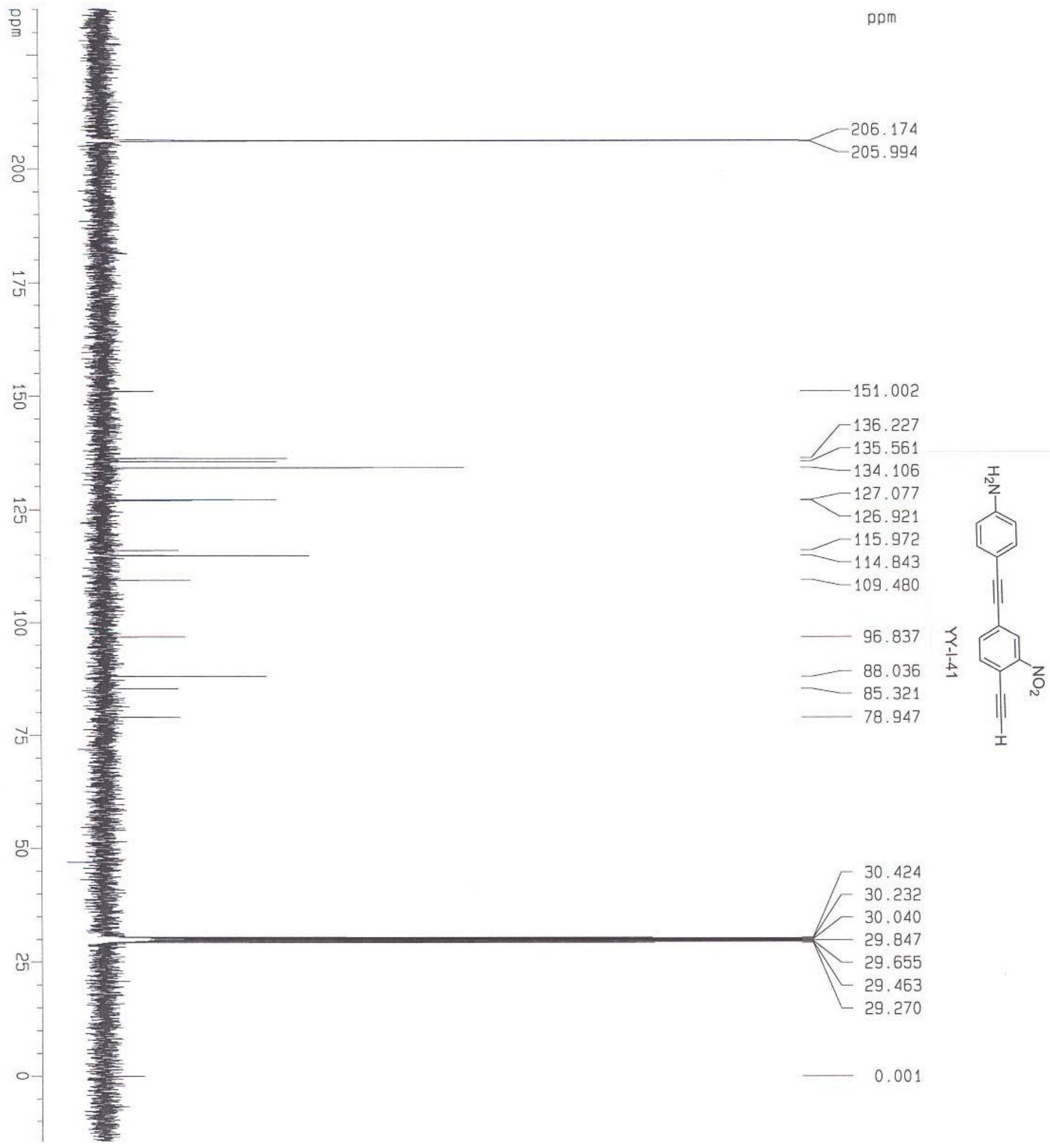

Figure S42: ${ }^{13} \mathrm{C}$ NMR of 25. 
Flatt, Yao, Maya, and Tour, Orthogonally Functionalized Oligomers, Supporting Information

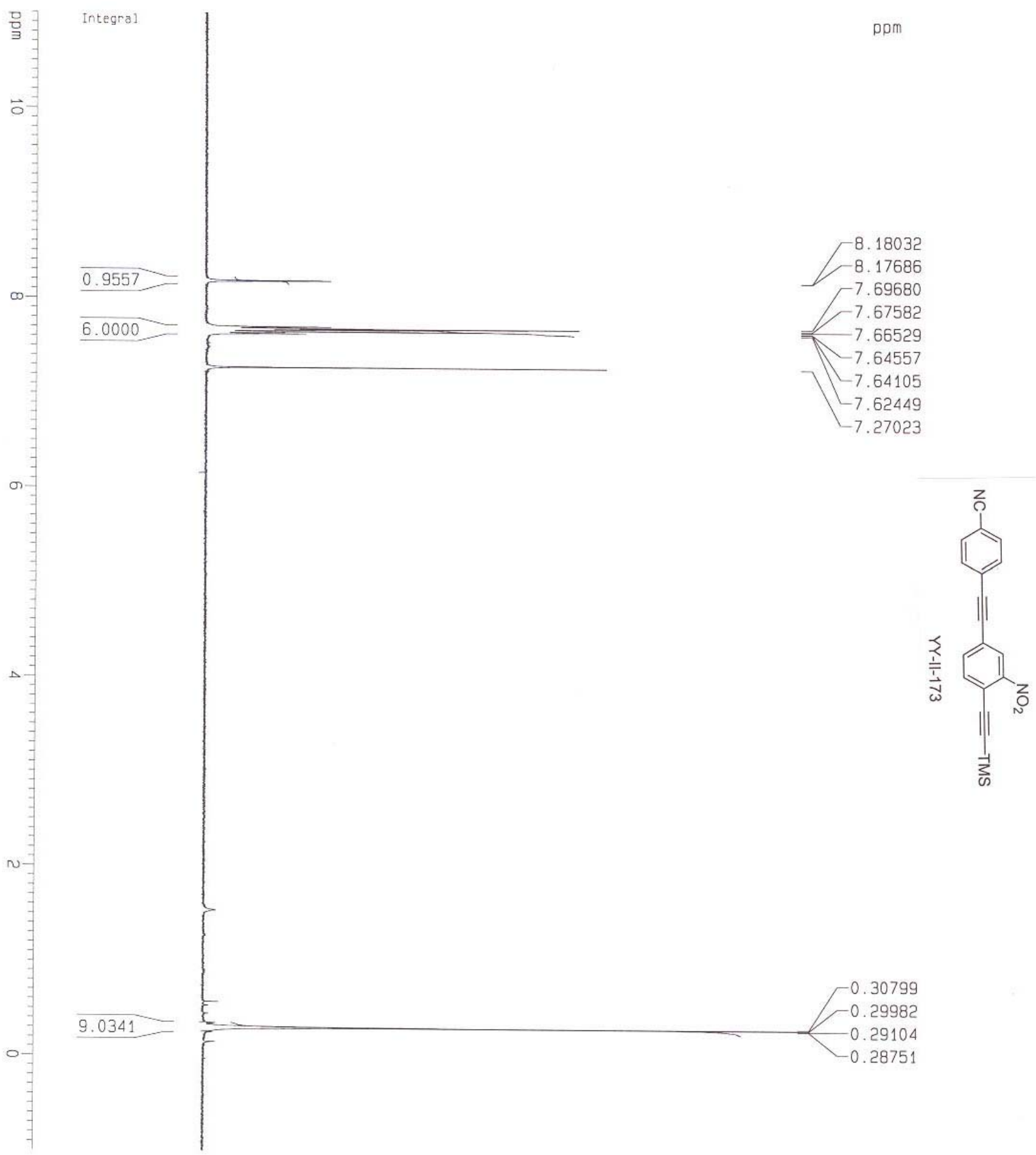

Figure S43: ${ }^{1} \mathrm{H}$ NMR of 27. 
Flatt, Yao, Maya, and Tour, Orthogonally Functionalized Oligomers, Supporting Information

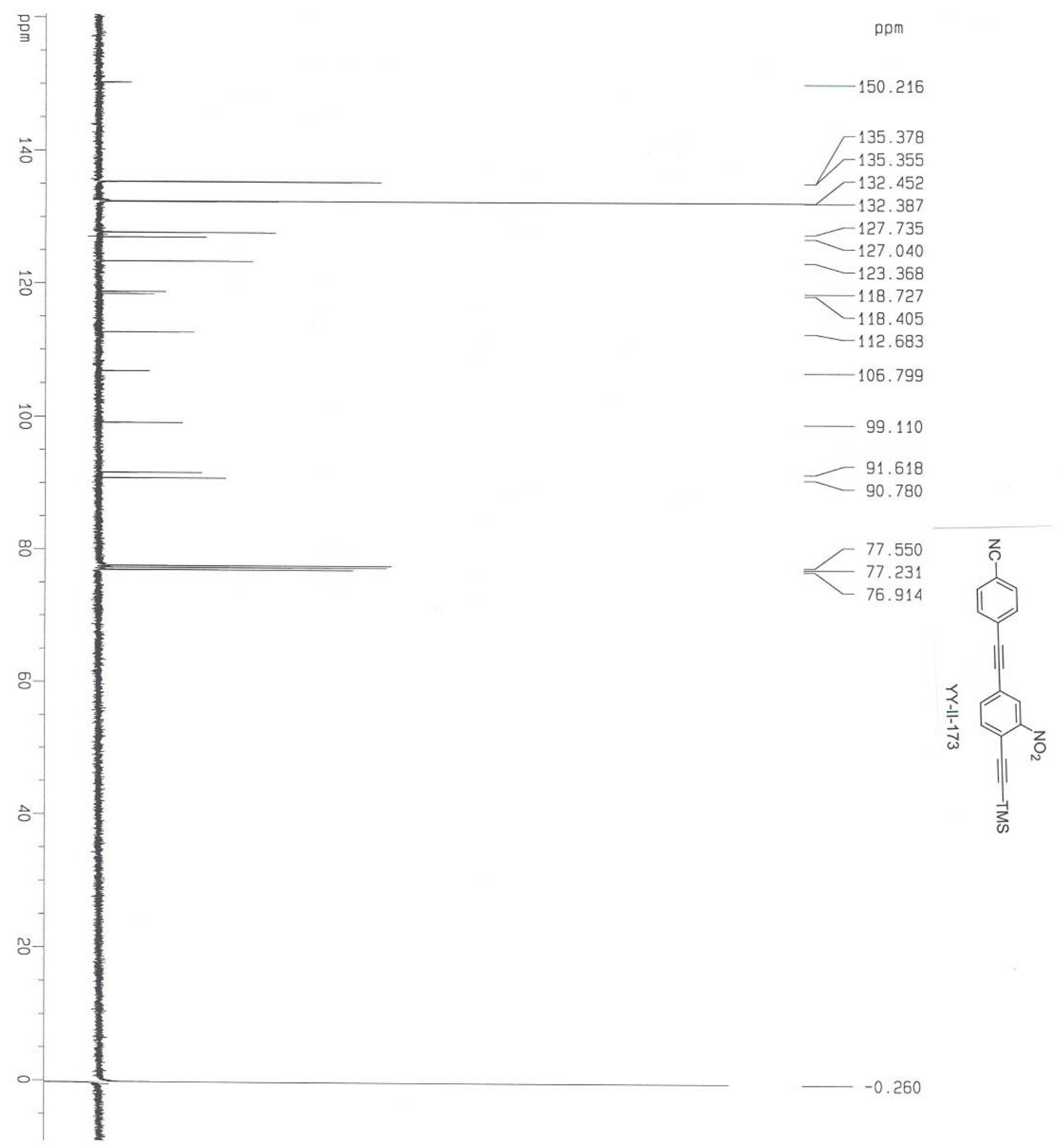

Figure S44: ${ }^{13} \mathrm{C}$ NMR of 27. 
Flatt, Yao, Maya, and Tour, Orthogonally Functionalized Oligomers, Supporting Information

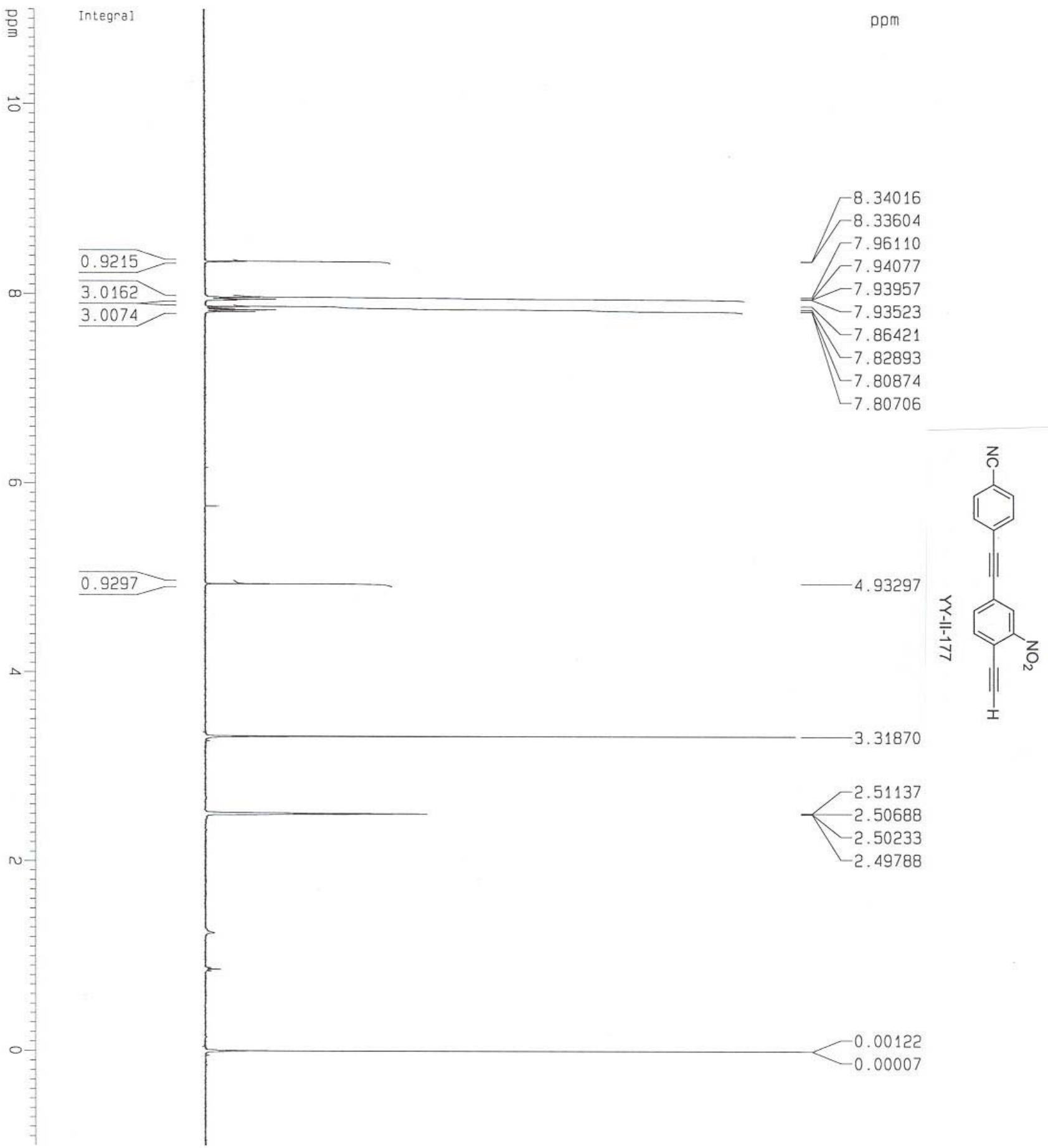

Figure S45: ${ }^{1} \mathrm{H}$ NMR of 28. 
Flatt, Yao, Maya, and Tour, Orthogonally Functionalized Oligomers, Supporting Information

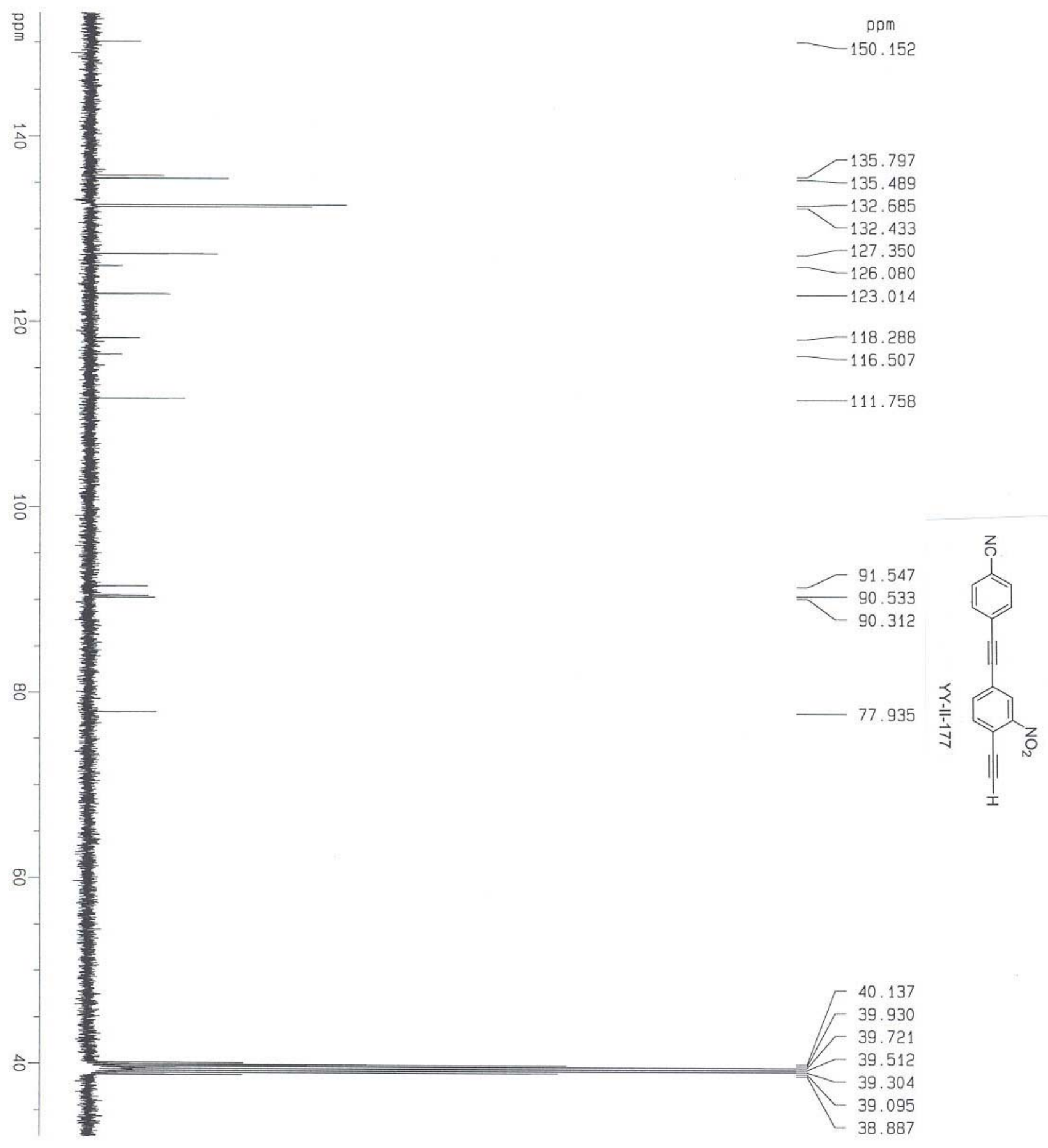

Figure S46: ${ }^{13} \mathrm{C}$ NMR of 28. 
Flatt, Yao, Maya, and Tour, Orthogonally Functionalized Oligomers, Supporting Information

\section{References (prior references on in main paper)}

${ }^{20}$ Wilson, G. J.; Hunt, F. C. Aust. J. Chem. 1983, 36, 2317-2326.

${ }^{19}$ (a) Sonogashira, K.; Tohda, Y.; Hagihara, N. Tetrahedron Lett. 1975, 16, 4467-4470. (b) Stephens, R. D.; Castro, C. E. J. Org. Chem. 1963, 28, 3313-3315. 